Universidade de São Paulo

Instituto de Física

\title{
Estados Coerentes e Seus Usos em Teorias de Campos em Espaços Curvos
}

\section{Rene Soares Freire}

Orientador: Prof. Dr. João Carlos Alves Barata

Dissertação apresentada ao Instituto de Física da Universidade de São Paulo para a obtenção do título de Mestre em Ciências.

\section{Banca Examinadora:}

Prof. Dr. André Gustavo Scagliusi Landulfo (CCNH/UFABC)

Prof. Dr. João Carlos Alves Barata (IF/USP)

Prof. Dr. Paulo Teotônio Sobrinho (IF/USP)

São Paulo 


\section{Sumário}

\begin{tabular}{lll}
\hline & Introdução & 1
\end{tabular}

\begin{tabular}{|lll}
2 & O Conteúdo Algébrico & 4
\end{tabular}

2.1 Conceitos Básicos . . . . . . . . . . . . . . . . 5

$2.1 .1 \quad$ Motivação para as CCR e CAR . . . . . . . . . . 5

2.1.2 O ponto de vista algébrico e a teoria de observáveis locais 11

2.1 .3 Estados . . . . . . . . . . . . . . . . . 14

2.2 Propriedades das CAR e CCR e suas álgebras . . . . . . . . . 22

$2.2 .1 \quad$ CAR e suas álgebras . . . . . . . . . . . . . . . 23

$2.2 .2 \quad$ CCR, a forma de Weyl e suas álgebras . . . . . . . . . 24

$\begin{array}{lll}3 & \text { O Conteúdo Relativístico } & 28\end{array}$

3.1 Revisão dos Conceitos Básicos . . . . . . . . . . . . . . . . . 29

3.1 .1 Distribuições em variedades . . . . . . . . . . . . . 37

3.1.2 $\quad$ Estrutural Causal . . . . . . . . . . . . . . . . . . . . 40

3.2 A Equação de Onda num Espaço-tempo Globalmente Hiperbólico 44

3.2.1 Formulações do Problema . . . . . . . . . . . . . . . 44

3.2 .2 Soluções Fundamentais . . . . . . . . . . . . . . . . . 46

3.2 .3 O Problema de Cauchy e Operadores de Green. . . . . 49

3.2 .4 Propriedades das Soluções . . . . . . . . . . . . . . 51

4 Quantização $\quad 55$

4.1 Axiomas de Haag-Kastler Generalizados . . . . . . . . . . . . 56

4.2 Quantização de Campos Escalares Livres . . . . . . . . . . . . 56

5 Estados Coerentes em Álgebras CCR 63

5.1 Relações de Incerteza . . . . . . . . . . . . . . . . . . . . . . 68

5.2 O Limite Clássico . . . . . . . . . . . . . . . . . . . . . . 71

$\begin{array}{llr}6 & \text { O Espaço de de Sitter } & 86\end{array}$

6.1 Equação de Onda e Quantização. . . . . . . . . . . . . . . . . . . 88

6.2 Limite Semi-Clássico . . . . . . . . . . . . . . . . . . . . . . . 91

6.3 Observações e Conclusão . . . . . . . . . . . . . . . . . . . . . 94 


\section{Resumo}

A questão de como sistemas quânticos correspondem a sistemas clássicos existe desde o surgimento da mecânica quântica e parece ser algo natural de se perguntar. E também desde o princípio da mecânica quântica estados coerentes são usados para responder esse tipo de questão, já que eles são, em certo sentido, os estados quânticos mais próximos a estados que descrevem sistemas clássicos. Seguindo os resultados de Hepp, que mostrou a correspondência tanto para o caso da mecânica quântica não relativística quanto para o caso de campos Bosônicos relativísticos, mostramos a correspondência entre sistemas Bosônicos livres em um espaço-tempo de de Sitter e soluções da equação de Klein-Gordon neste mesmo espaço.

Após introduzir os conceitos relevantes e construir a álgebra que descreve sistemas Bosônicos livres em um espaço globalmente hiperbólico, construímos estados coerentes para álgebras CCR na forma de Weyl e provamos o limite semi-clássico para uma região próxima à origem (ou, para um tempo fixo, em todo espaço de de Sitter). Além disso provamos que este limite independe do estado de vácuo - que, em geral, não é único.

\section{Abstract}

The question of the correspondence between quantum and classical systems is an issue since the beginnings of quantum mechanics and it seems like a natural question. Also since the start of quantum mechanics coherent states were used to answer this sort of question, since they are, in a sense, the quantum states closest to states that describe classical systems. Following the results of Hepp, who showed the correspondence for the case of nonrelativistic quantum mechanics as well as for relativistic Bosonic fields, we show the correspondence between free Bosonic field in a de Sitter space-time and the solutions of the Klein-Gordon equation in that same space-time.

After introducing the relevant concepts and the construction of the algebra that describes free Bosonic systems in a globally hyperbolic space-time, we construct coherent states for CCR algebras in Weyl's form, and we prove the semi-classical limit for a region close to the origin (or, for a fixed time, in the whole de Sitter space-time). We also prove that this limit is independent of the vacuum state - which might not be unique. 


\section{Introdução}

Essencialmente o proposito deste trabalho é achar uma correspondência entre entidades quânticas que representam sistemas bosônicos livres e campos não-quânticos em um espaço-tempo curvo.

As entidades quânticas que descrevem os campos lineares livres são álgebras $\mathscr{A}(\mathcal{O})$ que representam operações realizadas em uma região do espaço-tempo $\mathcal{O}$, o que se procura é, então, como passar de medidas dessas operações numa região $\mathcal{O}$ a soluções $u$ da equação de Klein-Gordon $\left(\square+m^{2}\right) u=\Upsilon^{1}$ definidas dentro da região $\mathcal{O}:^{2}$

Há, então, três eixos principais em torno dos quais este projeto se desenvolve: o primeiro diz respeito à formulação algébrica da teoria quântica; o segundo se refere às soluções de equações de campos em espaços-tempos curvos (e como definir entidades quânticas, à maneira algébrica, segundo esses campos nesses espaços-tempos); e por fim no terceiro ponto elaboramos o limite clássico usando estados coerentes (seguindo a definição de Yamagami [37]).

A formulação algébrica da teoria quântica de campos é útil para o que pretendemos fazer pois ela não assume uma teoria clássica subjacente, de modo que ela já nos fornece objetos quânticos, assumindo uma primazia da teoria quântica; e, ainda por outro lado, por isso ela é mas apropriada para uma generalização a espaços-tempos curvos. Ela foi primeiro estudada por I. Segal [31], que abstraiu da mecânica quântica as propriedades de operadores e estados, formulando-as em termos algébricos (assim abstraindo da mecânica quântica os espaços de Hilbert). Haag e Kastler [18] deram a forma final desta formulação, incluindo na abstração dos operadores a álgebras a formulação do principio de localidade.

As formulações tradicionais da teoria quântica de campos, por exemplo, a formulação através dos axiomas de Wightman [33], dependem fortemente não só do espaço de Hilbert (que é desejável se omitir, visto que há formulações da teoria em espaços de Hilbert que não são equivalente $\varsigma^{3}$ ) mas da simetria particular do espaço de Minkowski: nos axiomas de Wightman é exigido, por exemplo, que exista uma representação do grupo de Poincaré no espaço de Hilbert, que hajam domínios e vetores invariantes por esta representação, etc.

\footnotetext{
${ }^{1}$ Usaremos a métrica com a assinatura $(+,-,-,-)$.

${ }^{2}$ Apesar dessas soluções serem definidas globalmente para um espaço-tempo globalmente hiperbólico, não faria sentido essa equivalência ser dada em regiões do espaço-tempo que poderiam ser, em certo sentido, disjuntas.

${ }^{3}$ Para uma discussão elaborada deste tema, bem como as vantagens e limitações da teoria quântica de campos algébrica vide [29] e [19]; para uma interpretação do significado dessa não equivalência, vide 12
} 
A formulação algébrica, por outro lado, não se apoia tão fortemente sobre as particularidades do espaço-tempo subjacente (é fácil generalizar o axioma de Haag-Kastler que concerne o grupo de Poincaré, como o faz Dimock [13]). Para uma breve discussão destes temas, vide [35].

É natural, portanto, que para teorias quânticas de campos em um espaçotempo curvo (onde a gravidade é tratada de forma não-quântica) adotemos a formulação algébrica.

A generalização dos campos clássicos em espaços-tempos de Minkowski para espaços-tempos mais gerais é mais simples do que a quantização, já que basta trocarmos os operadores diferenciais definidos no espaço-tempo de Minkowski a operadores em espaços-tempos curvos (através das derivadas covariantes, etc.). As soluções destas equações em espaços-tempos curvos são regidas por uma teoria bem acabada mas extensa, de forma que fugiria completamente do escopo deste trabalho apresentá-la em detalhes; restringimo-nos, então, a enunciar os principais resultados e, de certa forma, indicar o caminho traçado para as provas gerais. Esta teoria foi primeiro formulada por Leray (em um trabalho que, para todos os propósitos, parece estar perdido ao mundo), e mais recentemente por Bär et al., por exemplo, em [2] e [3].

Sobre o limite clássico e estados coerentes: este tipo de correspondência foi uma preocupação constante desde o surgimento da própria mecânica quântica, e o uso de estados coerentes para fazer essa transição remonta à década de 20 do século XX, com o artigo de Schrödinger Der stetige Übergang von der Mikro- zur Makromechanik, "A transição continua da Microà Macro-mecânica" [30], no qual ele mostra que uma superposição dada por $\psi_{A}=\sum(A / 2)^{n} \psi_{n} / n$ ! das soluções do oscilador harmônico quântico $\psi_{n}$ (essencialmente funções de Hermite ${ }^{4}$ é precisamente uma das definições do estado coerente. Calculando essa soma Schrödinger mostra que $\psi_{A}$ é uma Gaussiana e que para $A \gg 1$ a largura da Gaussiana é muito pequena; e ainda que esse pacote de onda não se desfaça com a evolução temporal. Há assim uma correspondência — uma analogia, como diz Jammer [23] - entre a mecânica quântica e a mecânica clássica. ${ }^{5}$

A forma mais simples desta correspondência foi proposta por Ehrenfest [15], que nos mostrou que os valores esperados do momento $p$ e da posição $q$

\footnotetext{
${ }^{4} \mathrm{E}$ portanto, quando normalizadas, formam uma base ortonormal $\{|n\rangle\}$ do espaço de Hilbert $L^{2}$.

${ }^{5}$ Notemos ainda que o modo que Schrödinger formulou essa correspondência torna visível que de fato é absolutamente não trivial (mesmo se for algo esperado intuitivamente) que de fato haja tal correspondência, dada a disparidade de princípios entre a mecânica quântica e a mecânica clássica (por exemplo, principio da superposição).
} 
satisfazem equações que sugerem as equações de Hamilton:

$$
\frac{d}{d t}\langle q\rangle=\frac{1}{m}\langle p\rangle, \quad \frac{d}{d t}\langle p\rangle=-\left\langle V^{\prime}(q)\right\rangle .
$$

E para casos especiais (e.g., $V$ linear) $\left\langle V^{\prime}(q)\right\rangle=V^{\prime}(\langle q\rangle)$, e portanto uma genuína correspondência é estabelecida - de fato $(\langle q\rangle,\langle p\rangle)$ são soluções de um sistema clássico.

Com o uso de estados coerentes tanto o resultado de Ehrenfest quanto o resultado de Schrödinger são generalizados: para observáveis "centrados" em $\sqrt{\hbar} p$ e $\sqrt{\hbar} q$, calculados em estados de mínima incerteza obtemos soluções das equações de Hamilton, e portanto obtemos (1) com $\left\langle V^{\prime}(q)\right\rangle=V^{\prime}(\langle q\rangle)$. E ainda, provaremos (conforme provou antes Hepp [21]) que essa correspondência permanece quando os observáveis são evoluídos no tempo: ou seja, como sugere Schrödinger, o pacote de onda não se desfaz.

No capítulo (2) estudamos as motivações e os conceitos algébricos necessários para a formulação precisa dos axiomas de Haag-Kastler, bem como a sua formulação e o estudo da álgebra CCR (e esta álgebra será a álgebra em foco neste trabalho, já que ela pretende descrever sistemas bosônicos livres) inclusive a sua conformação aos axiomas supracitados. Na seção (4), imprecisamente nomeada Quantização, generalizamos os axiomas de Haag-Kastler, seguindo Dimock [13], para espaços-tempos globalmente hiperbólicos (esta condição é necessária pois só nestes espaços são garantidas soluções globais das equações clássicas), a construção da álgebra CCR (já que devemos especificar detalhes relativísticos, que são contidos na forma simplética que define a forma de Weyl destas álgebras) e a prova de que elas satisfazem os axiomas generalizados.

No capítulo (3) fazemos uma apresentação superficial da geometria diferencial, para fixar os conceitos e notações relevantes, bem como a apresentação da teoria das soluções das equações de ondas (mais especificamente, a solução da equação de Klein-Gordon) numa variedade Lorentziana.

Por fim, no capítulo (5) definimos os estados coerentes em álgebras CCR, e provamos que esses estados coerentes satisfazem a principal propriedade de estados coerentes, viz., que as incertezas da posição e momento são mínimas. Em 5.2 elaboramos mais sobre o limite semi-clássico e o provamos, seguindo [21, no caso mais simples.

No capítulo (6) aplicamos os resultados ao espaço-tempo de de Sitter e provamos o teorema principal. 
2 O Conteúdo Algébrico 


\subsection{Conceitos Básicos}

Antes de analisar as propriedades das álgebras geradas pelas relações canônicas de comutação e de anti-comutação faremos uma breve introdução, mostrando a motivação física de tais relações e porque estudar suas álgebras, introduzindo a formulação algébrica da física quântica, através dos axiomas de HaagKastler.

\subsubsection{Motivação para as CCR e CAR}

O que segue nesta seção são, em verdade, os primeiros passos que são tradicionalmente tomados (pelo lado matemático) para a segunda quantização; são, então, raciocínios bem conhecidos, de modo que os expressamos apenas para motivar definições e resultados posteriores. Explicitamos também algumas passagens que são em geral omitidas dos livros.

Seja $\mathscr{H}_{1}$ o espaço que representa o sistema de uma partícula. Para descrever um sistema de duas partículas não interagentes distinguívei ${ }^{6}$ podemos montar o espaço $\mathscr{H}_{2}$ da seguinte forma: $\mathscr{H}_{2}=\mathscr{H}_{1} \otimes \mathscr{H}_{1}$, e escrevemos um elemento de $\mathscr{H}_{2}$ como $\psi=\psi_{1} \otimes \psi_{2}{ }^{7}$. Analogamente para um sistema de $n$ partículas: $\mathscr{H}_{n}=\mathscr{H}_{1} \otimes \ldots \otimes \mathscr{H}_{1}$.

Se o número de partículas é variável, podemos montar um espaço formado pela soma direta dos $\mathscr{H}_{n}, n=0,1,2, \ldots$, onde $\mathscr{H}_{0}=\mathbb{C}$.

Agora, o seguinte espaço é chamado de um espaço de Fock:

$$
\begin{gathered}
\mathscr{H}_{F}=\bigoplus_{n=0}^{\infty} \mathscr{H}_{n}, \quad \mathscr{H}_{0}=\mathbb{C}, \\
\|\psi\|^{2}=\sum_{n=0}^{\infty}\left\|\psi_{n}\right\|^{2}<\infty, \quad \psi \in \mathscr{H}_{F}, \psi_{n} \in \mathscr{H}_{n} .
\end{gathered}
$$

Elementos de $\mathscr{H}_{F}$ são sequencias: $\mathscr{H}_{F} \ni \psi=\left(\psi_{0}, \psi_{1}, \psi_{2}, \ldots\right)$. Com a norma indicada, afirmamos que $\mathscr{H}_{F}$ é um espaço de Banach. Que a soma acima de fato define uma norma é elementar, então, vou mostrar que $\mathscr{H}_{F}$ é completo: Considere a sequencia $\left(\psi^{l}\right)_{l} \subset \mathscr{H}_{F}$ uma sequencia de Cauchy: $\psi^{l}=\left(\psi_{0}^{l}, \psi_{1}^{l}, \ldots\right)$, $\forall \varepsilon>0, \exists N>0$ tal que $\left\|\psi^{l}-\psi^{k}\right\|<\varepsilon, \forall l, k>N$. Então (como esses termos são positivos podemos elevar ao quadrado sem problemas),

$$
\left\|\psi^{l}-\psi^{k}\right\|^{2}=\sum_{n}\left\|\psi_{n}^{l}-\psi_{n}^{k}\right\|^{2}<\varepsilon \Rightarrow\left\|\psi_{n}^{l}-\psi_{n}^{k}\right\|<\varepsilon
$$

\footnotetext{
${ }^{6}$ Mais à frente aplicaremos operadores de (anti-)simetrização para o tratamento de partículas não interagentes idênticas.

${ }^{7} \mathrm{O}$ produto interno em $\mathscr{H}_{1} \otimes \mathscr{H}_{1}$ é: $\left(\psi_{1} \otimes \psi_{2}, \phi_{1} \otimes \phi_{2}\right)=\left(\psi_{1}, \phi_{1}\right)\left(\psi_{2}, \phi_{2}\right)$
} 
Então, $\left(\psi_{k}^{l}\right)_{l} \subset \mathscr{H}_{k}$ é uma sequencia de Cauchy, e como $\mathscr{H}_{k}$ é completo, $\left(\psi_{k}^{l}\right)_{l}$ converge, digamos, para $\psi_{k}$. Tomamos, então, como Ansatz para o possível vetor para o qual $\psi^{l}$ converge a seguinte sequência: $\psi=\left(\psi_{0}, \psi_{1}, \ldots\right)$, formada pelos $\psi_{k}$ tais que $\psi_{k}^{l} \rightarrow \psi_{k}$. Basta mostrar que $\psi \in \mathscr{H}_{F}$ e que $\psi^{l} \rightarrow \psi$.

Tome agora um $l$ suficientemente grande de tal forma que $\left\|\psi_{n}-\psi_{n}^{l}\right\|$ seja desprezável, então, escrevemos $\left\|\psi_{n}\right\|$ da seguinte forma: $\left\|\psi_{n}\right\|=\| \psi_{n}+\psi_{n}^{l}-$ $\psi_{n}^{l}\|\leq\| \psi_{n}-\psi_{n}^{l}\|+\| \psi_{n}^{l} \|$, e da forma que escolhemos $l$ (ato que sempre podemos realizar) concluímos que $\left\|\psi_{n}\right\|^{2} \leq\left\|\psi_{n}^{l}\right\|^{2}$, então, claramente, $\psi \in$ $\mathscr{H}_{F}$. É simples ver que $\psi^{l} \rightarrow \psi$, já que $\psi$ foi construído para que isso desse certo. De fato, a norma da diferença é a raiz da soma das diferenças de cada termo de $\psi^{l}$ e $\psi$, e este converge àquele.

O espaço $\mathscr{H}_{F}$ é também um espaço de Hilbert, cujo produto interno é definido por

$$
(\psi, \phi)=\sum_{n}\left(\psi_{n}, \phi_{n}\right), \quad \psi_{n}, \phi_{m} \in \mathscr{H}_{n} .
$$

Que esta expressão de fato define um produto interno é imediato.

Um exemplo de espaço de Fock, que é considerado frequentemente na mecânica quântica, é aquele no qual $\mathscr{H}_{1}=L^{2}\left(\mathbb{R}^{3}\right)$, nesse caso $\mathscr{H}_{1} \ni \psi=\psi(\mathbf{x}), \mathbf{x} \in \mathbb{R}^{3}$, e $\mathscr{H}_{2} \ni \psi_{2}=\psi_{2}\left(\mathbf{x}_{1}, \mathbf{x}_{2}\right)$, etc.

Para encontrarmos as desejadas CCR e CAR (canonical commutation relations e canonical anti-commutation relations, respectivamente) devemos entender como operadores agem no espaço de Fock $\mathscr{H}_{F}$, e então considerar dois subespaços de $\mathscr{H}_{F}$, que são construídos devido a considerações de indistinguibilidade entre partículas. Com isso, construiremos dois operadores, um par em cada subespaço, que funcionarão como criadores e aniquiladores de partículas.

Primeiro, definiremos os subespaços $\mathscr{H}_{F}^{ \pm}$, os espaços de Fock simetrizados e antisimetrizados, da seguinte forma: sejam os operadores $\Pi^{ \pm}: \mathscr{H}_{n} \rightarrow \mathscr{H}_{n}{ }^{8}$ que agem da seguinte forma:

$$
\Pi^{ \pm}\left(\psi_{1} \otimes \ldots \otimes \psi_{n}\right)=\frac{1}{n !} \sum_{\sigma} \epsilon_{\sigma}^{ \pm} \psi_{\sigma(1)} \otimes \ldots \otimes \psi_{\sigma(n)},
$$

onde $\sigma$ são permutações de $n$ índices, e $\epsilon_{\sigma}^{-}$é +1 se $\sigma$ for uma permutação par e -1 se $\sigma$ for uma permutação impar, e $\epsilon_{\sigma}^{+}=1$. Esses operadores ainda têm as seguintes propriedades: $\left(\Pi^{ \pm}\right)^{2}=\Pi^{ \pm}$e $\left(\Pi^{ \pm}\right)^{*}=\Pi^{ \pm}$, e ainda para $\psi \in \mathscr{H}_{n}$,

\footnotetext{
${ }^{8}$ Mais precisamente, defino antes $\Pi^{ \pm}$em um subconjunto denso de $\mathscr{H}_{n}$, a saber, o conjunto formado por combinações finitas de $\psi_{1} \otimes \ldots \otimes \psi_{n}$, que forma uma base de $\mathscr{H}_{n}$ (prova em [27]), e como $\Pi^{ \pm}$é limitado podemos estendê-lo para todo $\mathscr{H}_{n}$.
} 
$\left\|\Pi^{ \pm} \psi\right\| \leq\|\psi\|$ (prova em [14])

Definimos, então, os subespaços $\mathscr{H}_{n}^{ \pm}$de $\mathscr{H}_{n}$ por $\mathscr{H}_{n}^{ \pm}=\Pi^{ \pm} \mathscr{H}_{n}$. Os espaços de Fock simétrico e anti-simétrico $\mathscr{H}_{F}^{ \pm}$são:

$$
\mathscr{H}_{F}^{ \pm}=\bigoplus_{n=0}^{\infty} \mathscr{H}_{n}^{ \pm} .
$$

Outros subespaços importantes são os formados por sequencias de $\mathscr{H}_{F}$ e $\mathscr{H}_{F}^{ \pm}$ que tem seus elementos $\psi$ com $\psi_{n} \neq 0$ apenas para uma quantidade finita de $n$. Esses subespaços são denotados por $D_{0}$ e $D_{0}^{ \pm}$, e são densos em $\mathscr{H}_{F}$ e $\mathscr{H}_{F}^{ \pm}$. De fato, para algum $\psi \in \mathscr{H}_{F}, \psi=\left(\psi_{0}, \psi_{1}, \ldots\right)$, tomo a sequencia $\left(\phi^{l}\right)_{l} \subset D_{0}$, $\phi^{l}=\left(\psi_{0}, \psi_{1}, \ldots, \psi_{l}, 0,0, \ldots\right)$. É facil ver que para todo $\varepsilon$ podemos encontrar um $l$ suficientemente grande tal que $\left\|\phi^{l}-\psi\right\|<\varepsilon$. Analogamente para os demais casos.

Agora, mostraremos como a partir de operadores definidos em subespaços densos de $\mathscr{H}_{n}$ podemos definir operadores no espaço de Fock (e isso implica que um operador definido num espaço de uma partícula pode ser estendido de forma natural para todo o espaço de Fock). Para isso, veremos como construir operadores em um espaço que é produto tensorial a partir de operadores definidos nos espaços que formam o primeiro espaço. Quando há dois operadores, digamos, $A$ e $B$ densamente definidos, com domínios $D_{A}$ e $D_{B}{ }^{9}$, em $\mathscr{H}_{A}$ e $\mathscr{H}_{B}$. Definimos o operador $A \otimes B: D_{A} \otimes D_{B} \rightarrow \mathscr{H}_{A} \otimes \mathscr{H}_{B}$, por: $\forall \phi \otimes \psi \in D_{A} \otimes D_{B}$

$$
(A \otimes B)(\phi \otimes \psi)=(A \phi) \otimes(B \psi) .
$$

Notemos que $D_{A} \otimes D_{B}$ é denso em $\mathscr{H}_{A} \otimes \mathscr{H}_{B}$, e se $A$ e $B$ forem fecháveis, então, $A \otimes B$ e $A \otimes \mathbb{1}+\mathbb{1} \otimes B$ são também fecháveis (prova em [27]). Com essas definições e resultados podemos definir operadores em $\mathscr{H}_{n}$ a partir de operadores definidos em $\mathscr{H}_{1}$, da seguinte forma: seja $A$ um operadores autoadjunto, com domínio de essencial auto-adjuntabilidade $D$ (i.e., $A$ é em $D$ essencialmente auto-adjunto), definimos $d \Gamma(A): \mathcal{D}_{A} \rightarrow \mathscr{H}$ por

$$
\begin{gathered}
\mathcal{D}_{A}=\left\{\psi \in D_{0}: \psi_{n} \in \bigotimes_{k=1}^{n} D, \text { para cada } n,\right\} \\
\left.d \Gamma(A)\right|_{\mathcal{D}_{A} \cap \mathscr{H}_{n}}=A \otimes \mathbb{1} \otimes \cdots \otimes \mathbb{1}+ \\
+\mathbb{1} \otimes A \otimes \mathbb{1} \otimes \cdots \otimes \mathbb{1}+\cdots+\mathbb{1} \otimes \mathbb{1} \otimes \cdots \otimes \mathbb{1} \otimes A .
\end{gathered}
$$

$d \Gamma(A)$ é essencialmente auto-adjunto em $\mathcal{D}_{A}$ (Teorema VIII.33 de [27]).

\footnotetext{
${ }^{9}$ Para definições e elaborações sobre operadores não-limitados (como, por exemplo, a definição de densamente definido ou de operadores fecháveis) vide Capítulo 39 de [4]
} 
Exemplo Tomemos $A=\mathbb{1}$. Nesse caso, $D_{\mathbb{1}}=\mathscr{H}$, então, $\mathcal{D}_{\mathbb{1}}=\{\psi \in$ $\left.D_{0}: \psi_{n} \in \mathscr{H}_{n}\right\}=D_{0}$, e, dado $\mathscr{H}_{n} \ni \psi=\psi_{1} \otimes \cdots \otimes \psi_{n}$ temos:

$$
\left.d \Gamma(\mathbb{1})\right|_{\mathcal{D}_{\mathbb{1}} \cap \mathscr{H}_{n}} \psi=\mathbb{1} \psi_{1} \otimes \cdots \otimes \mathbb{1} \psi_{n}+\cdots+\mathbb{1} \psi_{1} \otimes \cdots \otimes \mathbb{1} \psi_{n}=n \psi .
$$

Ou seja, para $\psi \in D_{0},(d \Gamma(\mathbb{1}) \psi)_{n}=n \psi_{n}$. O operador $d \Gamma(\mathbb{1})$ é chamado de operador número de partículas $N$. É imediato que $N$ é um operador positivo, e, portanto, podemos definir $\sqrt{N}(\mathrm{e} \sqrt{N+1})([28])$. Se estendermos $N$ para todo $\mathscr{H}_{F}$ é simples ver que se deve exigir o domínio:

$$
D_{N}=\left\{\psi \in \mathscr{H}_{F}: \sum n^{2}\left\|\psi_{n}\right\|^{2}<\infty\right\}
$$

Antes de definir os operadores de criação e aniquilação definimos operadores auxiliares $b(f)$ e $b^{*}(f)$, em $D_{0}$, para cada $f \in \mathscr{H}_{1}$ da seguinte forma:

$$
\begin{aligned}
b(f) \psi_{0} & =0, \quad b^{*}(f) \psi_{0}=f, \\
b(f)\left(\psi_{1} \otimes \cdots \otimes \psi_{n}\right) & =\sqrt{n}\left(f, \psi_{1}\right)\left(\psi_{2} \otimes \cdots \otimes \psi_{n}\right), \\
b^{*}(f)\left(\psi_{1} \otimes \cdots \otimes \psi_{n}\right) & =\sqrt{n+1}\left(f \otimes \psi_{1} \otimes \cdots \otimes \psi_{n}\right) .
\end{aligned}
$$

Lembrando da desigualdade de Cauchy-Schwarz $\left|\left(f, \psi_{1}\right)\right| \leq\|f\|\left\|\psi_{1}\right\|$, calculamos que $\|b(f) \psi\| \leq n^{1 / 2}\|f\|\|\psi\|$, e $\left\|b^{*}(f) \psi\right\|=(n+1)^{1 / 2}\|f\|\|\psi\|$. Então podemos estender $b(f)$ e $b^{*}(f)$ para $\mathscr{H}_{F}$.

Note ainda que $b^{*}(f)$ é o adjunto de $b(f)$, pois, chamando $\psi=f_{1} \otimes \cdots \otimes f_{n}$ e $\phi=g_{1} \otimes \cdots \otimes g_{n}$, calculamos:

$$
\begin{gathered}
(\phi, b(f) \psi)=\sum_{n}\left(f, f_{1}\right)(n+1)^{1 / 2}\left(g_{1} \otimes \cdots \otimes g_{n}, f_{2} \otimes \cdots \otimes f_{n+1}\right) \\
=\sum_{n}\left((n+1)^{1 / 2} f \otimes g_{1} \otimes \cdots \otimes g_{n}, f_{1} \otimes \cdots \otimes f_{n+1}\right),
\end{gathered}
$$

onde as somas acima são finitas. Impondo a igualdade dessa soma com $\left(b^{*}(f) \phi, \psi\right)=\sum_{k}\left(\left(b^{*}(f) \phi\right)_{k}, \psi_{k}\right)$, e lembrando que $D_{0}$ é denso em $\mathscr{H}_{F}$, concluímos que esse $b^{*}(f)$ é idêntico ao operador definido mais acima.

Os operadores de aniquilação e criação são: $a_{ \pm}(f)=\Pi^{ \pm} b(f)$ e $a_{ \pm}^{*}(f)=$ $\Pi^{ \pm} b^{*}(f)$. E $a_{ \pm}^{*}(f)$ é o adjunto de $a_{ \pm}(f)$, pois, para $\phi, \psi \in D_{0}, a_{ \pm}(f)$ e $a_{ \pm}^{*}(f)$ agem em $\Pi^{ \pm} \psi$ e $\Pi^{ \pm} \phi$. Então:

$$
\begin{aligned}
\left(\Pi^{ \pm} \phi, \Pi^{ \pm} b(f) \Pi^{ \pm} \psi\right)=\left(\Pi^{ \pm} \Pi^{ \pm} \phi, b(f) \Pi^{ \pm} \psi\right) & =\left(b^{*}(f) \Pi^{ \pm} \phi, \Pi^{ \pm} \psi\right)= \\
& =\left(\Pi^{ \pm} b^{*}(f) \Pi^{ \pm} \phi, \Pi^{ \pm} \psi\right) .
\end{aligned}
$$


E também valem as relações, para $\psi \in \mathscr{H}_{n}^{ \pm},\left\|a_{ \pm}(f) \psi\right\| \leq n^{1 / 2}\|f\|\|\psi\|$ e $\left\|a_{ \pm}(f)^{*} \psi\right\| \leq(n+1)^{1 / 2}\|f\|\|\psi\|$. A primeira relação segue imediatamente do que foi mostrado para $b(f)$, a segunda é confirmada pelo seguinte cálculo:

$$
\left\|a_{ \pm}^{*}(f) \psi\right\|=\left\|\Pi^{ \pm} b^{*}(f) \psi\right\| \leq\left\|b^{*}(f) \psi\right\|=(n+1)^{1 / 2}\|f\|\|\psi\| .
$$

São esses operadores que em $\mathscr{H}_{F}^{ \pm}$satisfazem as relações canônicas de comutação e de anti-comutação, que são as seguintes:

$$
\begin{gathered}
{\left[a_{+}(f), a_{+}(g)\right]=\left[a_{+}^{*}(f), a_{+}^{*}(g)\right]=0,} \\
{\left[a_{+}(f), a_{+}^{*}(g)\right]=(f, g) \mathbb{1},} \\
\left\{a_{-}(f), a_{-}(g)\right\}=\left\{a_{-}^{*}(f), a_{-}^{*}(g)\right\}=0, \\
\left\{a_{-}(f), a_{-}^{*}(g)\right\}=(f, g) \mathbb{1},
\end{gathered}
$$

onde, $[A, B]=A B-B A$ e $\{A, B\}=A B+B A$.

Para prová-las usaremos seguintes formas mais explicitas para $a_{ \pm}(f)$ e $a_{ \pm}^{*}(f)$ [14: para $\psi_{1} \otimes \cdots \otimes \psi_{n}$ em $\mathscr{H}_{n}, \Pi^{ \pm}\left(\psi_{1} \otimes \cdots \otimes \psi_{n}\right)$, que está em $\mathscr{H}_{n}^{ \pm}$, é um elemento tipico no qual $a_{ \pm}(f)$ e $a_{ \pm}^{*}(f)$ agem,

$$
a_{ \pm}^{*}(f) \Pi^{ \pm}\left(\psi_{1} \otimes \cdots \otimes \psi_{n}\right)=\sqrt{n+1} \Pi^{ \pm}\left(f \otimes \psi_{1} \otimes \cdots \otimes \psi_{n}\right)
$$

$\mathrm{e}$

$$
a_{ \pm}(f) \Pi^{ \pm}\left(\psi_{1} \otimes \cdots \otimes \psi_{n}\right)=\frac{1}{\sqrt{n}} \sum_{j=1}^{n}( \pm 1)^{j+1}\left(f, \psi_{j}\right) \Pi^{ \pm}\left(\psi_{1} \otimes \cdots \otimes \hat{\psi}_{j} \otimes \cdots \otimes \psi_{n}\right),
$$

onde o circunflexo em $\psi_{j}$ indica sua omissão no produto. Delas verificamos:

$$
\begin{gathered}
a_{ \pm}(f) a_{ \pm}^{*}(g) \Pi^{ \pm}\left(\psi_{1} \otimes \cdots \otimes \psi_{n}\right)=\sqrt{n+1} a_{ \pm}(f) \Pi^{ \pm}\left(g \otimes \psi_{1} \otimes \cdots \otimes \psi_{n}\right) \\
=(f, g) \Pi^{ \pm}\left(\psi_{1} \otimes \cdots \otimes \psi_{n}\right)+\sum_{j=1}^{n}( \pm 1)^{j}\left(f, \psi_{j}\right) \Pi^{ \pm}\left(g \otimes \psi_{1} \otimes \cdots \otimes \hat{\psi}_{j} \otimes \cdots \otimes \psi_{n}\right) .
\end{gathered}
$$

Por outro lado:

$$
\begin{aligned}
& a_{ \pm}^{*}(g) a_{ \pm}(f) \Pi^{ \pm}\left(\psi_{1} \otimes \cdots \otimes \psi_{n}\right)= \\
& =\frac{1}{\sqrt{n}} a_{ \pm}^{*}(g) \sum_{j=1}^{n}( \pm 1)^{j+1}\left(f, \psi_{j}\right) \Pi^{ \pm}\left(\psi_{1} \otimes \cdots \otimes \hat{\psi}_{j} \otimes \cdots \otimes \psi_{n}\right)= \\
& =\sum_{j=1}^{n}( \pm 1)^{j+1}\left(f, \psi_{j}\right) \Pi^{ \pm}\left(g \otimes \psi_{1} \otimes \cdots \otimes \hat{\psi}_{j} \otimes \cdots \otimes \psi_{n}\right) .
\end{aligned}
$$


Comparando esses dois resultados concluímos as relações canônicas.

A interpretação física dos operadores $a_{ \pm}(f)$ e $a_{ \pm}^{*}(f)$ é que quando eles atuam num estado com $n$ partículas o primeiro o transforma num estado de $n-1$ partículas, destruindo uma partícula no estado $f$, enquanto o segundo o transforma num estado de $n+1$ partículas, criando uma partícula no estado $f$. Essa interpretação se sugere da própria ação que $a_{ \pm}(f)$ e $a_{ \pm}^{*}(f)$ têm sobre estados de $n$ partículas.

Seja $\Omega=(1,0,0, \ldots) \in \mathscr{H}_{F}^{ \pm}$o vácuo, aplicando $a_{ \pm}^{*}(f)$ é criada uma partícula no estado $f: \psi_{ \pm}(f)=a_{ \pm}^{*}(f) \Omega$. Aplicando sucessivamente:

$$
\begin{aligned}
& \psi_{ \pm}\left(f_{1}, \cdots, f_{n}\right)=a_{ \pm}^{*}\left(f_{1}\right) \otimes \cdots \otimes a_{ \pm}^{*}\left(f_{n}\right) \Omega=a_{ \pm}^{*}\left(f_{1}\right) \otimes \cdots \otimes a_{ \pm}^{*}\left(f_{n-1}\right) \Pi^{ \pm} f_{n} \\
= & \sqrt{2} a_{ \pm}^{*}\left(f_{1}\right) \otimes \cdots \otimes a_{ \pm}^{*}\left(f_{n-2}\right) \Pi^{ \pm}\left(f_{n-1} \otimes f_{n}\right)=\ldots=\sqrt{n !} \Pi^{ \pm}\left(f_{1} \otimes \cdots \otimes f_{n}\right) .
\end{aligned}
$$

E ainda: se $\left\{f_{k}\right\}$ é uma base ortonormal de $\mathscr{H}_{1}$ conjuntos da forma $\left\{f_{1} \otimes \cdots \otimes\right.$ $\left.f_{n}\right\}$, para todo $n$, formam um conjunto denso em $\mathscr{H}_{F}$ quando são percorridos por subconjuntos finitos de $\left\{f_{k}\right\}$. Ou seja, aplicando o operador de criação no vácuo obtemos uma base de $\mathscr{H}_{F}^{ \pm}$(e podemos através de combinações lineares construir qualquer estado de $\mathscr{H}_{F}^{ \pm}$). De fato, isso foi tomado como um axioma por Wightman, mas no contexto dos axiomas de Wightman não é exigido que o espaço de Hilbert seja um espaço de Fock (portanto, a interpretação é a mesma que a dada aqui somente para o caso em que o espaço de Hilbert é um espaço de Fock).

Exemplos [14] Para duas partículas distinguíveis não-interagentes sem spin sabemos que o hamiltoniano é $H=-\Delta_{1} / 2 m_{1}-\Delta_{2} / 2 m_{2}$, que age sobre $\psi\left(\mathbf{x}_{1}, \mathbf{x}_{2}\right), \mathbf{x}_{1}, \mathbf{x}_{2} \in \mathbb{R}^{3}$.

Usando as definições dadas durante essa seção diríamos que o estado $\psi$ pertence à $L^{2}\left(\mathbb{R}^{3}\right) \otimes L^{2}\left(\mathbb{R}^{3}\right) \simeq L^{2}\left(\mathbb{R}^{6}\right)$, e o hamiltoniano é dado por

$$
H=H_{1} \otimes \mathbb{1}+\mathbb{1} \otimes H_{2},
$$

onde $H_{i}=-\Delta / 2 m_{i}, i=1,2$. Então,

$$
H=\frac{-1}{2 m_{1}} \Delta \otimes \mathbb{1}+\mathbb{1} \otimes \frac{-1}{2 m_{2}} \Delta=\frac{-1}{2 m_{1}} \Delta_{1}+\frac{-1}{2 m_{2}} \Delta_{2} .
$$

Para $n$ bósons ou férmions não interagentes o espaço que contém os estados puros é

$$
\Pi^{ \pm} L^{2}\left(\mathbb{R}^{3}\right) \otimes \cdots \otimes L^{2}\left(\mathbb{R}^{3}\right) \simeq \Pi^{ \pm} L^{2}\left(\mathbb{R}^{3 n}\right) .
$$


Ou seja, os estados são representados por funções $\Pi^{ \pm} \psi\left(\mathbf{x}_{1}, \ldots, \mathbf{x}_{n}\right), \mathbf{x}_{k} \in \mathbb{R}^{3}$. E o hamiltoniano é (sabendo que $H=-\Delta / 2 m$ ), em $D_{n}$,

$$
d \Gamma(H)=H \otimes \mathbb{1} \otimes \cdots \otimes \mathbb{1}+\cdots+\mathbb{1} \otimes \cdots \otimes H=\sum_{i=1}^{n} \frac{-1}{2 m} \Delta_{i} .
$$

\subsubsection{O ponto de vista algébrico e a teoria de observáveis locais}

Definições Uma álgebra complexa $\mathscr{A}$ é um espaço vetorial sobre os complexos com um produto . : $\mathscr{A} \times \mathscr{A} \rightarrow \mathscr{A}$, distributivo em relação à soma e à multiplicação por escalares - e como de costume omitimos o ponto: $a . b=$ $a b \in \mathscr{A}, \forall a, b \in \mathscr{A} \mathbb{1 0}$. Uma *álgebra é uma álgebra na qual existe uma bijeção $\mathscr{A} \ni a \mapsto a^{*} \in \mathscr{A}$ que satisfaz:

$\left(a^{*}\right)^{*}=a, \quad(a b)^{*}=b^{*} a^{*}, \quad(\alpha a+\beta b)^{*}=\bar{\alpha} a^{*}+\bar{\beta} b^{*}, \quad \forall a, b \in \mathscr{A}, \forall \alpha, \beta \in \mathbb{C}$.

Uma $C^{*}$-álgebra é uma *álgebra com uma norma completa ${ }^{11}$, i.e., $\|\|:. \mathscr{A} \rightarrow$ $\mathbb{R}$, tal que

$$
\begin{gathered}
\|a\| \geq 0, \quad \text { e, } \quad\|a\|=0 \Leftrightarrow a=0 \\
\|a+b\| \leq\|a\|+\|b\|, \quad\|a b\| \leq\|a\|\|b\|, \quad\|\alpha a\|=|\alpha|\|a\|,
\end{gathered}
$$

tal que

$$
\left\|a^{*} a\right\|=\|a\|^{2}
$$

Um ${ }^{*}$-morfismo entre duas *-álgebras $\mathscr{A}_{1}$ e $\mathscr{A}_{2}$ é uma aplicação $\pi: \mathscr{A}_{1} \rightarrow \mathscr{A}_{2}$ que satisfaz:

$$
\begin{gathered}
\pi(\alpha a+b)=\alpha \pi(a)+\pi(b), \\
\pi(a b)=\pi(a) \pi(b), \\
\pi\left(a^{*}\right)=\pi(a)^{*} .
\end{gathered}
$$

Uma representação de uma álgebra $\mathrm{C}^{*} \mathscr{A}$ em um espaço de Hilbert $\mathscr{H}$ é o $\operatorname{par}(\mathscr{H}, \pi)$, onde $\pi: \mathscr{A} \rightarrow B(\mathscr{H})$ é um *-morfismo entre $\mathscr{A}$ e $B(\mathscr{H})$, onde $B(\mathscr{H})$ é o conjunto dos operadores lineares limitados de $\mathscr{H}$.

Dizemos que duas representações $\left(\mathscr{H}_{1}, \pi\right),\left(\mathscr{H}_{2}, \phi\right)$ são equivalentes se existe um operador unitário $U: \mathscr{H}_{1} \rightarrow \mathscr{H}_{2}$ tal que $U \pi(a)=\phi(a) U, \forall a \in \mathscr{A}$.

\footnotetext{
${ }^{10}$ Assumimos por todo o texto que o produto é associativo.

${ }^{11} \mathrm{Ou}$ seja, toda sequência de Cauchy é uma sequência convergente.
} 
Um *-isomorfismo entre duas álgebras é um *-morfismo bijetor. Portanto, se $\pi$ é um *-isomorfismo entre duas álgebras então ker $\pi=\{0\}$ (pela linearidade de $\pi$ ). Uma representação de $\mathscr{A}$ em $\mathscr{H}$ é fiel se o ${ }^{*}$-morfismo for um *-isomorfismo. Uma representação fiel preserva a norma: $\|\pi(a)\|=\|a\|$. (Proposição 2.3.3 de [9]).

Um conjunto $B$ de operadores limitados em $\mathscr{H}$ é (algebricamente) ${ }^{12}$ irredutivel se os únicos subespaços de $\mathscr{H}$ invariantes ${ }^{13}$ por $B$ é $\{0\}$ e $\mathscr{H}$. Posso falar também em representações (algebricamente) irredutíveis: uma representação $\pi$ de $\mathscr{A}$ em $\mathscr{H}$ é irredutível se os únicos subespaços de $\mathscr{H}$ invariantes por $\pi(\mathscr{A})$ forem $\{0\}$ e $\mathscr{H}$.

Uma álgebra $\mathscr{A}$ é primitiva se ela possuir uma representação irredutível e fiel.

Rede de Álgebras e Estrutura Quasi-Local A partir de uma rede de álgebras $\left\{\mathscr{A}_{i}\right\}_{i \in I}$ construímos uma álgebra $\mathscr{A}$, e se essas álgebras formarem uma certa estrutura, chamamos $\mathscr{A}$ de uma álgebra quasi-local. Para definir álgebras quasi-locais antes devemos definir uma relação de ortogonalidade no conjunto ordenado e dirigidd ${ }^{14}$ de índices $I$, da seguinte forma: $I$ possui uma ortogonalidade se há uma relação $\perp$ em $I$ que satisfaz:

(i) $i \in I \Rightarrow \exists j \in I$ tal que $i \perp j$;

(ii) $i \leq j$ e $j \perp k$, então, $i \perp k$;

(iii) $i \perp j$ e $i \perp k$, então, $\exists m \in I$ tal que $i \perp m$ e $m \geq j, k$.

Exemplos Um exemplo importante para aplicações na mecânica estatística é aquele no qual $I$ consiste de conjuntos limitados e abertos em $\mathbb{R}^{n}$, ordenados por inclusão, e a relação de ortogonalidade é: $i \perp j \Leftrightarrow i \cap j=\emptyset$. As duas primeiras relações são fáceis de serem verificadas, para a relação (iii) basta notar que podemos tomar $m=j \cup k$, então, $i \cap m=i \cap(j \cup k)=(i \cap j) \cup(i \cap k)=\emptyset$ e $m \supseteq j, k$.

O exemplo de interesse para a formulação algébrica da teoria quântica de campos é o exemplo no qual $I$ consiste de conjuntos abertos limitados no espaço de Minkowski (i.e., regiões finitas do 4-dimensional espaço-tempo),

\footnotetext{
${ }^{12}$ Há também o conceito de topologicamente irredutível, no qual é inserido a condição dos subespaços invariantes serem fechados; mas segundo 9 para álgebras $\mathrm{C}^{*}$ irredutibilidade algébrica e topológica se implicam.

${ }^{13}$ Um subespaço $B \subset A$ é invariante por um operador $P$ (ou conjunto de operadores) se $P B \subset B$.

${ }^{14} \mathrm{Ou}$ seja, $\forall a, b \in I, \exists c$ tal que $c>a$ e $c>b$.
} 
ordenados por inclusão, com a relação $i \perp j \Leftrightarrow i$ e $j$ são separados espacialmente (i.e., se para $x \in i$ e $y \in j$ temos $\left.(x-y)^{2}<0\right){ }^{15}$. Essa relação claramente satisfaz as condições de ortogonalidade, sendo que mais uma vez podemos tomar, para o item (iii), $m=j \cup k$.

Definição de álgebra quasi-local Dada uma rede de $C^{*}$-álgebras $\left\{\mathscr{A}_{i}\right\}_{i \in I}$, com o conjunto de índices $I$ possuidor de uma relação de ortogonalidade, uma álgebra quasi-local é uma $\mathrm{C}^{*}$-álgebra $\mathscr{A}$ se:

(1) $i \geq j \Rightarrow \mathscr{A}_{i} \supseteq \mathscr{A}_{j}$;

(2) As álgebras $\mathscr{A}_{i}$ possuem uma identidade $\mathbb{1}$ em comum;

(3) $i \perp j \Leftrightarrow\left[\mathscr{A}_{i}, \mathscr{A}_{j}\right]=0$;

(4) $\mathscr{A}=\overline{\bigcup_{i} \mathscr{A}_{i}}$.

Observações: No item (4) é exigido que $\mathscr{A}$ seja o fecho da união, pois a união claramente gera uma *álgebra, e com o fecho temos uma $\mathrm{C}^{*}$-álgebra. Podemos ainda incluir o caso em que os elementos de $\mathscr{A}_{i}$ anticomutam, modificando a condição (3) para: existe um automorfismo $\sigma$, tal que $\sigma^{2}=1 \mathrm{e}$ $\sigma\left(\mathscr{A}_{i}\right)=\mathscr{A}_{i}$, e vale que: $\left[\mathscr{A}_{i}^{e}, \mathscr{A}_{j}^{e}\right]=\left[\mathscr{A}_{i}^{e}, \mathscr{A}_{j}^{o}\right]=\left\{\mathscr{A}_{i}^{o}, \mathscr{A}_{j}^{o}\right\}=0$ sempre que $i \perp j$. Onde $\mathscr{A}_{i}^{e}$ e $\mathscr{A}_{i}^{o}$ são as partes pares e impares de $\mathscr{A}_{i}$. A definição de partes pares e impares de $\mathscr{A}_{i}$ vem da seguinte observação: dado um automorfismo $\sigma$ de uma álgebra $\mathscr{A}$ tal que $\sigma^{2}=1$ podemos decompor $a \in \mathscr{A} \mathrm{em}$ $a=a^{+}+a^{-}, \operatorname{com} a^{ \pm}=(1 / 2)(a \pm \sigma(a)), a^{+}=a^{e}$ e $a^{-}=a^{o}$. As relações canônicas de anti-comutação são recuperadas quando $\sigma(a)=-a$, pois, nesse caso $a^{o}=a$ e $a^{e}=0$. Apesar dessa inclusão de casos de álgebras com elementos que anti-comutam, Haag observa em [17] que no contexto da formulação algébrica da teoria quântica a imposição de comutação das álgebras quando as regiões são espacialmente separadas é mais um reflexo da estrutura causal do espaço-tempo do que um reflexo da estatística de Bose. De fato, nessa formulação a relação entre uma região do espaço-tempo com uma álgebra determina os observáveis de um sistema.

Axiomas de Haag-Kastler Com essas definições os axiomas de HaagKastler [18] são reescritos da seguinte forma: há uma correspondência entre

\footnotetext{
${ }^{15}$ Para a formulação (de J. Dimock [13]) mais geral no contexto da relatividade geral substitui-se o espaço de Minkowski pela variedade lorentziana $(\mathscr{M}, g)$, orientada temporalmente, com a ortogonalidade sendo ainda se duas regiões finitas $i$ e $j$ de $\mathscr{M}$ são separadas espacialmente (i.e., não há uma curva causal (ou seja, uma curva cujos vetores tangentes não são tipo-tempo nem nulo) que una pontos de $i$ e $j$ )
} 
regiões e álgebras:

$$
\mathcal{O} \mapsto \mathscr{A}(\mathcal{O})
$$

tal que:

(1) As regiões para as quais a relação (2) é definida correspondem à conjuntos abertos com fecho compacto num espaço de Minkowski. A coleção dessas regiões forma um conjunto de índices com a ortogonalidade $\mathcal{O}_{1} \perp \mathcal{O}_{2} \Leftrightarrow \mathcal{O}_{1}$ e $\mathcal{O}_{2}$ são separados espacialmente. As álgebras $\{\mathscr{A}(\mathcal{O})\}$ obedecem à estrutura que defina uma álgebra quasi-local e, portanto, definem uma álgebra quasilocal $\mathscr{A}$.

(2) O grupo não homogêneo de Lorent $\sqrt{16} \mathcal{P}_{+}^{\uparrow}$ é representado por automorfismos em $\mathscr{A}$ tais que, para $\mathcal{P}_{+}^{\uparrow} \ni g=(\Lambda, a), \alpha_{g}(\mathscr{A}(\mathcal{O}))=\mathscr{A}(g \mathcal{O})$, onde $g \mathcal{O}=\{\Lambda x+a, \quad x \in \mathcal{O}\}$

(3) $\mathscr{A}$ é primitivo.

\subsubsection{Estados}

Nesta seção faremos um pequeno estudo do conceito de estado (e, apesar de não ser o foco da seção, mencionarei observáveis).

Antes de definir estado no contexto da formulação algébrica da física quântica descreveremos brevemente a teoria geral dos estados e observáveis, tal como se encontra no capítulo 1 de Araki [1]. Essa descrição se não é importante para nós, é ao menos interessante, permitindo-nos preencher um pouco mais a (possível) lacuna que separa o mundo real observado através dos seus fenômenos e sua expressão matemática.

Ao realizar um experimento podemos abstrair do processo todo duas partes importantes (sabendo que existem outras), a saber, o objeto que será medido e o instrumento usado na medição. A medição é a interação entre essas duas partes. Denotemos os objetos medidos por $\alpha_{1}, \alpha_{2}$, etc., e os instrumentos por $Q_{1}, Q_{2}$, etc. A interação deve gerar números reais $p, q$, etc. Por exemplo, podemos medir $\alpha$ usando $Q$ e obter $q$.

No entanto, é sabido que ao se realizar outras medidas em $\alpha$ usando $Q$ (ou seus equivalentes) podemos obter outros resultados. Seja $N$ o número de medidas realizadas, e dentro dessas medidas seja $n_{q}$ a quantidade de vezes

\footnotetext{
${ }^{16}$ Tomamos $\mathcal{P}_{+}^{\uparrow}$ que é o grupo de Poincaré próprio ortócrono, cujos elementos têm determinante igual a um e $\Lambda_{0}^{0} \geq 1$.
} 
que obtemos $q$. Então, a probabilidade de se medir $q$ em $\alpha$ usando $Q$ é

$$
w_{\alpha}^{Q}(q)=\lim _{N \rightarrow \infty}\left(n_{q} / N\right) .
$$

Essa função claramente obedece as condições usuais para distribuições de probabilidades. Os estados de um sistema são os elementos do conjunto $\Sigma$ das classes de equivalência $[\alpha]$, com a equivalência $\alpha_{1} \sim \alpha_{2} \Leftrightarrow w_{\alpha_{1}}^{Q}(q)=$ $w_{\alpha_{2}}^{Q}(q), \forall Q, \forall q$.

Definimos também a equivalência entre dois instrumentos quando $Q_{1} \sim$ $Q_{2} \Leftrightarrow w_{\alpha}^{Q_{1}}(q)=w_{\alpha}^{Q_{2}}(q), \forall \alpha, \forall q$. A coleção de todas as classes de equivalência entre instrumentos é o conjunto $\mathscr{A}$, cujos elementos chamamos de observáveis.

Há ainda uma forma mais útil de caracterizar estados, a partir dos valores esperados de um observável $Q$. O valor esperado de um observável $Q$ num estado $\alpha$ é o número $\alpha(Q)$ dado por

$$
\alpha(Q)=\sum_{q} q w_{\alpha}^{Q}(q)
$$

E a coleção dos valores esperados para todos os $Q$ contém à mesma informação que o conjunto das probabilidades $w_{\alpha}^{Q}(q)$, de tal forma que podemos considerar $\alpha(Q)$ como o objeto caracterizador dos estados.

Agora, para passar para uma estrutura matemática mais concreta: dizemos que os observáveis podem ser representados por elementos de uma $\mathrm{C}^{*}$-álgebra $\mathscr{A}$ e os estados podem ser representados por funcionais em $\mathscr{A}$ lineares positivos normalizados, i.e., $\psi: \mathscr{A} \rightarrow \mathbb{C}$ tais que $\psi(\alpha a+b)=\alpha \psi(a)+\psi(b), \forall a, b \in$ $\mathscr{A}, \forall \alpha \in \mathbb{C}, \psi\left(a^{*} a\right) \geq 0$ e $\|\psi\|=\sup \left\{\psi\left(a^{*} a\right),\|a\|=1\right\}=1$. Na proposição 2.3.11 de [9] é garantido que $\psi$ é continuo (e, portanto, existe a norma) apenas garantindo que ele seja linear e positivo.

Note que isso se reduz à usual formulação da mecânica quântica se tomarmos $\mathscr{A}$ como sendo o conjunto dos operadores lineares auto-adjuntos num espaço de Hilbert $\mathscr{H}$ e

$$
\psi(A)=\left(\phi_{\psi}, A \phi_{\psi}\right)
$$

Estados assim são chamados de estados vetoriais. Em verdade: é sempre possível, dado um estado $\psi$, escrevê-lo na forma (3) de forma única, graças à construção GNS (Gelfand-Naimark-Segal).

Antes de apresentar o teorema que enuncia precisamente e garante a construção GNS vejamos alguns fatos sobre estados. Primeiro, a desigualdade de Cauchy-Schwarz:

$$
\left|\psi\left(a^{*} b\right)\right|^{2} \leq \psi\left(a^{*} a\right) \psi\left(b^{*} b\right) .
$$


Tomando $c=z a-b, z \in \mathbb{C}, a, b \in \mathscr{A}$, então,

$$
\begin{gathered}
0 \leq \psi\left(c^{*} c\right)=\psi\left(\bar{z} z a^{*} a-\bar{z} a^{*} b-z b^{*} a+b^{*} b\right)= \\
=|z|^{2} \psi\left(a^{*} a\right)-\bar{z} \psi\left(a^{*} b\right)-z \psi\left(b^{*} a\right)+\psi\left(b^{*} b\right) .
\end{gathered}
$$

Para ver as formas das condições para que isso se satisfaça tomemos $z=z_{1}+$ $i z_{2}, z_{1}, z_{2} \in \mathbb{R}$, então o termo do meio é simplesmente $-i z_{2}\left(\psi\left(b^{*} a\right)-\psi\left(a^{*} b\right)\right)$, e para que a desigualdade valha devemos ter, no mínimo, que $\left(\psi\left(b^{*} a\right)-\right.$ $\left.\psi\left(a^{*} b\right)\right) \notin \mathbb{R}$, e, portanto, $\psi\left(b^{*} a\right)=\overline{\psi\left(a^{*} b\right)}$. Então,

$$
|z|^{2} \psi\left(a^{*} a\right)-2 \Re\left(z \psi\left(a^{*} b\right)\right)+\psi\left(b^{*} b\right) \geq 0 .
$$

Agora, para achar outra condição tomamos - supondo que $\psi\left(a^{*} b\right) \neq 0-$ $z=\psi\left(b^{*} b\right) / \psi\left(a^{*} b\right)$. Portanto,

$$
\psi\left(a^{*} a\right) \frac{\psi\left(b^{*} b\right)^{2}}{\left|\psi\left(a^{*} b\right)\right|^{2}}-\psi\left(b^{*} b\right) \geq 0 \Rightarrow\left|\psi\left(a^{*} b\right)\right| \leq \psi\left(a^{*} a\right) \psi\left(b^{*} b\right) .
$$

Durante a prova da desigualdade de Cauchy-Schwarz descobrimos o fato geral

$$
\psi\left(a^{*} b\right)=\overline{\psi\left(b^{*} a\right)}
$$

que também é importante. Tomando $b=\mathbb{1}$ obtemos ainda $\psi\left(a^{*}\right)=\overline{\psi(a)}$. Substituindo $b=\mathbb{1}$ na desigualdade de Cauchy-Schwarz: $|\psi(a)|^{2} \leq \psi\left(a^{*} a\right)\|\psi\|$.

Faremos uma breve pausa no fluxo do texto para introduzir um conceito crucial na teoria das $\mathrm{C}^{*}$-álgebras, viz., o conceito de elementos positivos de uma $\mathrm{C}^{*}$-álgebra, provando alguns resultados ligados a essa ideia.

Seja a $C^{*}$-álgebra $\mathscr{A}$. $a \in \mathscr{A}$ é um elemento positivo de $\mathscr{A}$ se $a$ for autoadjunt $^{17}$ e se $\sigma(a) \subseteq \mathbb{R}^{+} 18$ O conjunto dos elementos positivos de $\mathscr{A}$ é denotado por $\mathscr{A}^{+}$. Agora, em uma de suas formas o teorema espectral diz que se $a \in \mathscr{A}$ é auto-adjunto e $f \in C(\sigma(a))$-onde $C(A)$ é o conjunto das funções continuas em $A$-então, $\sigma(f(a))=\{f(t): t \in \sigma(a)\}$. Temos o seguinte resultado:

$$
f(a) \in \mathscr{A}^{+} \Leftrightarrow f(t) \geq 0, \forall t \in \sigma(a) .
$$

Pois, se $f(a) \in \mathscr{A}^{+}$então $\sigma(f(a)) \subseteq \mathbb{R}^{+}$, mas $\sigma(f(a))=\{f(t): t \in \sigma(a)\}$, então, $\{f(t): t \in \sigma(a)\} \subseteq \mathbb{R}^{+}$, ou seja, $f(t) \geq 0, \forall t \in \sigma(a)$. Por outro lado, se $f(t) \geq 0, \forall t \in \sigma(a)$ significa que $\mathbb{R}^{+} \supseteq\{f(t): t \in \sigma(a)\}=\sigma(f(a))$,

\footnotetext{
${ }^{17}$ Isto é, se $a^{*}=a$.

${ }^{18} \mathrm{Um}$ número complexo $\lambda$ esta no resolvente de $a, r(a)$, se $(\lambda \mathbb{1}-a)$ for inversível. Se $\lambda$ não esta no resolvente de $a$ então $\lambda$ esta no espectro de $a, \sigma(a)$.
} 
portanto, $f(a) \in \mathscr{A}^{+}$.

Tomando $f \in C(\sigma(a)), f(t)=\|a\| \pm t$, para $a \in \mathscr{A}^{+} . f(t) \geq 0$ para $t \in \sigma(a)$, então, $f(a)=\|a\| \mathbb{1} \pm a \in \mathscr{A}^{+}$.

Um fato importante sobre elementos positivos é que eles podem ser escritos da forma

$$
a \in \mathscr{A}^{+} \Leftrightarrow a=b^{*} b, \text { para algum } b \in \mathscr{A} .
$$

Por ora não provarei isso, mas assumindo sua validade tomemos para $a \in \mathscr{A}^{+}$ o elemento $c=\left(a^{1 / 2} b\right)^{*} a^{1 / 2} b,{ }^{19}$ que pertence a $\mathscr{A}^{+}$pois ele é da forma (5). Mas, $c=b^{*} a b$. Resumindo, $a \in \mathscr{A}^{+}, b \in \mathscr{A} \Rightarrow b^{*} a b \in \mathscr{A}^{+}$. Note também que (5) implica que $\psi(a) \geq 0, \forall a \in \mathscr{A}^{+}$.

Com esses resultados em mãos farei uma conta que será importante na prova do teorema da construção GNS: $\|a\|^{2} \mathbb{1}-a^{*} a=\left\|a^{*} a\right\| \mathbb{1}-a^{*} a \in \mathscr{A}^{+}$, então, $b^{*}\left(\|a\|^{2} \mathbb{1}-a^{*} a\right) b \in \mathscr{A}^{+}$. Portanto,

$$
\begin{gathered}
0 \leq \psi\left(b^{*}\left(\|a\|^{2} \mathbb{1}-a^{*} a\right) b\right)=\|a\|^{2} \psi\left(b^{*} b\right)-\psi\left(b^{*} a^{*} a b\right) \\
\therefore \psi\left(b^{*} a^{*} a b\right) \leq\|a\|^{2} \psi\left(b^{*} b\right) .
\end{gathered}
$$

Provemos, agora, o seguinte teorema:

Teorema 2.1. Seja $\psi$ um estado sobre a $C^{*}$-álgebra $\mathscr{A}$, então existem: um espaço de Hilbert $\mathscr{H}_{\psi}$, uma representação $\pi_{\psi}$ de $\mathscr{A}$ em $\mathscr{H}_{\psi}$ (i.e., um *morfismo entre $\mathscr{A}$ e $B\left(\mathscr{H}_{\psi}\right)$ ) e um vetor unitário $\Omega_{\psi} \in \mathscr{H}_{\psi}$, que satisfazem:

(1) $\forall a \in \mathscr{A}, \quad \psi(a)=\left(\Omega_{\psi}, \pi_{\psi}(a) \Omega_{\psi}\right)$;

(2) $\pi_{\psi}(\mathscr{A}) \Omega_{\psi}=\left\{\pi_{\psi}(a) \Omega_{\psi}: a \in \mathscr{A}\right\}$ é denso em $\mathscr{H}_{\psi}$.

E tal que o terno $\left(\mathscr{H}_{\psi}, \pi_{\psi}, \Omega_{\psi}\right)$ é único, a não ser por uma equivalência unitária.

Observações: A ultima proposição do teorema significa o seguinte: se existir outro terno $\left(\mathscr{H}_{\psi}^{\prime}, \pi_{\psi}^{\prime}, \Omega_{\psi}^{\prime}\right)$ que satisfaz (1) e (2), então existe uma aplicação unitária $U: \mathscr{H}_{\psi} \rightarrow \mathscr{H}_{\psi}^{\prime}$ tal que

$$
U \pi_{\psi}(a)=\pi_{\psi}^{\prime}(a) U, \forall a \in \mathscr{A}, \text { e } U \Omega_{\psi}=\Omega_{\psi}^{\prime} .
$$

Essa construção nos garante uma conexão mais clara com a interpretação probabilística da física quântica (que é melhor enxergada em estados na forma vetorial), enquanto ainda preserva as vantagens da formulação algébrica, como,

\footnotetext{
${ }^{19}$ Usei o fato de que se $a \in \mathscr{A}^{+}$então existe um $a^{1 / 2} \in \mathscr{A}$ tal que $a=\left(a^{1 / 2}\right)^{2}$
} 
por exemplo, a independência da formulação à um espaço de Hilbert em particular.

Demonstração. A prova será dividida em quatro partes: na primeira parte construiremos o espaço de Hilbert $\mathscr{H}_{\psi}$, a partir de $\mathscr{A}$, usando $\psi$ na definição do produto interno. Na segunda parte construímos o $\pi_{\psi}$. Na terceira parte definimos o $\Omega_{\psi}$ e na quarta parte mostramos a unicidade do terno $\left(\mathscr{H}_{\psi}, \pi_{\psi}, \Omega_{\psi}\right)$.

(i) Seja $L_{\psi}=\left\{a \in \mathscr{A}: \psi\left(a^{*} a\right)=0\right\}$. Primeiro, considere $L_{\psi}^{\prime}=\{a \in$ $\left.\mathscr{A}: \psi\left(a^{*} b\right)=0, \forall b \in \mathscr{A}\right\}$. Note que como $\psi\left(a^{*} b\right)=\overline{\psi\left(b^{*} a\right)}, L_{\psi}^{\prime}$ é igual à $\left\{a \in \mathscr{A}: \psi\left(b^{*} a\right)=0, \forall b \in \mathscr{A}\right\}$. É claro que $L_{\psi}^{\prime} \subset \mathrm{E}_{\psi}$.

Mas, $a \in L_{\psi} \Rightarrow a \in L_{\psi}^{\prime}$, pois, para $a \in L_{\psi},\left|\psi\left(a b^{*}\right)\right|^{2} \leq \psi\left(a^{*} a\right) \psi\left(b^{*} b\right)=0 \Rightarrow$ $\psi\left(a b^{*}\right)=0$. Então, $L_{\psi} \subset L_{\psi}^{\prime}$. Portanto, $L_{\psi}=L_{\psi}^{\prime}$.

E além disso $L_{\psi}$ é um subespaço linear de $\mathscr{A}$ e é um ideal esquerdo, ou seja, se $l \in L_{\psi}$ e $a \in \mathscr{A}$ implica em al $\in L_{\psi}$. Pois, seja $l \in L_{\psi}$ e $a \in \mathscr{A}$, então, $\psi\left((a l)^{*} b\right)=\psi\left(l^{*} a^{*} b\right)=0, \forall b \in \mathscr{A} \Rightarrow a l \in L_{\psi}$. A prova de que $L_{\psi}$ é um subespaço de $\mathscr{A}$ é elementar: sejam $l_{1}, l_{2} \in \mathscr{A}$ e $\alpha \in \mathbb{C}$, então, $\psi\left(\left(\alpha l_{1}+l_{2}\right)^{*} a\right)=\bar{\alpha} \psi\left(l_{1}^{*} a\right)+\psi\left(l_{2}^{*} a\right)=0 \Rightarrow \alpha l_{1}+l_{2} \in L_{\psi}$.

Considere agora o conjunto $D_{\psi}=\mathscr{A} / L_{\psi}$, formado pelas classes de equivalência definidas pela equivalência $a \sim a^{\prime} \Leftrightarrow a-a^{\prime} \in L_{\psi}$. O elemento neutro de $D_{\psi}$ é o $L_{\psi}$ inteiro. Com as seguintes operações $D_{\psi}$ é um espaço vetorial: $[a]+[b]=[a+b]$ e $\alpha[a]=[\alpha a]$.

Seja a operação $():, D_{\psi} \times D_{\psi} \rightarrow \mathbb{C}$ dada por $([a],[b])=\psi\left(a^{*} b\right)$. Antes de tudo é preciso mostrar que essa operação não depende do representante $a$ da classe de equivalência $[a]$. Ou seja, devemos mostrar que se $a \sim a^{\prime}$ e $b \sim b^{\prime}$ então $\psi\left(a^{\prime *} b^{\prime}\right)=\psi\left(a^{*} b\right)$. Mas,

$$
a \sim a^{\prime} \Leftrightarrow \exists l_{1} \in L_{\psi} \text { tal que } l=a-a^{\prime} \therefore a^{\prime}=a+l_{1} .
$$

Da mesma forma para $b \sim b^{\prime}, b^{\prime}=b+l_{2}$. Então,

$$
\begin{gathered}
\psi\left(\left(a+l_{1}\right)^{*}\left(b+l_{2}\right)\right)=\psi\left(\left(a^{*}+l_{1}^{*}\right)\left(b+l_{2}\right)\right) \\
=\psi\left(a^{*} b\right)+\psi\left(a^{*} l_{2}\right)+\psi\left(l_{1}^{*} b\right)+\psi\left(l_{1}^{*} l_{2}\right)=\psi\left(a^{*} b\right) .
\end{gathered}
$$

Então (, ) esta bem definido. Vou chamar os elementos de $D_{\psi}$ por $[a]=\xi_{a}$, $[b]=\xi_{b}$, etc.

Agora, (, ) é um produto interno em $D_{\psi}$, pois, $\left(\xi_{a}, \xi_{a}\right)=\psi\left(a^{*} a\right) \geq 0$ e 
$\psi\left(a^{*} a\right)=0 \Leftrightarrow a \in L_{\psi}$, ou seja, se e somente se $\xi_{a}=0$, e $\left(\xi_{a}, \xi_{b}\right)=\psi\left(a^{*} b\right)=$ $\overline{\psi\left(b^{*} a\right)}=\overline{\left(\xi_{b}, \xi_{a}\right)}$. E por ultimo,

$$
\begin{array}{r}
\left(\xi_{a}, \alpha \xi_{b}+\xi_{c}\right)=\left(\xi_{a}, \xi_{\alpha b+c}\right)=\psi\left(a^{*}(\alpha b+c)\right)=\alpha \psi\left(a^{*} b\right)+\psi\left(a^{*} c\right)= \\
=\alpha\left(\xi_{a}, \xi_{b}\right)+\left(\xi_{a}, \xi_{c}\right) .
\end{array}
$$

Então $D_{\psi}$ é um espaço pre-Hilbert, e basta completá-lo para obtermos o espaço de Hilbert $\mathscr{H}_{\psi}$.

O procedimento de completação de um espaço pre-Hilbert é algo que já deve ser assumido como conhecido, mas mostraremos aqui esse procedimento, seguindo a estrutura que se encontra em [38]. O espaço $\mathscr{H}_{\psi}$ será aquele formado por classes de equivalências das sequências de Cauchy em $D_{\psi}$. De forma mais precisa: Sejam $\xi_{a}=\left\{\xi_{a}^{n}\right\}_{n}$ e $\xi_{b}=\left\{\xi_{b}^{n}\right\}_{n}$ duas sequências de Cauchy. Define-se a relação de equivalência $\xi_{a} \sim \xi_{b} \Leftrightarrow \lim _{n \rightarrow \infty}\left\|\xi_{a}^{n}-\xi_{b}^{n}\right\|=0$. De fato isso é uma relação de equivalência: $\xi_{a} \sim \xi_{b} \Leftrightarrow \lim _{n \rightarrow \infty}\left\|\xi_{a}^{n}-\xi_{b}^{n}\right\|=0=\lim _{n \rightarrow \infty} \| \xi_{b}^{n}-$ $\xi_{a}^{n} \| \Leftrightarrow \xi_{b} \sim \xi_{a} ;$ se $\xi_{a} \sim \xi_{b}$ e $\xi_{b} \sim \xi_{c} \Leftrightarrow \lim \left\|\xi_{a}^{n}-\xi_{b}^{n}\right\|=0$ e $\lim \left\|\xi_{b}^{n}-\xi_{c}^{n}\right\|=0$, então, $\lim \left\|\xi_{a}^{n}-\xi_{c}^{n}\right\| \leq \lim \left(\left\|\xi_{a}^{n}-\xi_{b}^{n}\right\|+\left\|\xi_{b}^{n}-\xi_{c}^{n}\right\|\right)=0$ Então, $\xi_{a} \sim \xi_{c}$. Que $\xi_{a} \sim \xi_{a}$ é elementar.

$\mathrm{O}$ conjunto formado por tais classes de equivalência será chamado de $\mathscr{H}_{\psi}$. A soma e multiplicação por escalares são definidos da forma usual, e como soma e multiplicação por escalares de sequências de Cauchy são ainda sequências de Cauchy, está tudo bem definido. Chamemos os elementos de $\mathscr{H}_{\psi},\left[\xi_{a}\right],\left[\xi_{b}\right]$ de $\xi_{a}, \xi_{b}$, etc. Agora, define-se o produto interno em $\mathscr{H}_{\psi}$ por

$$
\left(\xi_{a}, \xi_{b}\right)=\lim _{n \rightarrow \infty}\left(\xi_{a}^{n}, \xi_{b}^{n}\right)
$$

Antes de tudo é preciso mostrar que essa operação está bem definida, ou seja, que ela não depende do particular representante das classes $\xi_{a}$, etc. Seja, então, $\xi_{a} \sim \xi_{a}^{\prime}$ e $\xi_{b} \sim \xi_{b}^{\prime}$, então:

$$
\begin{aligned}
& \mid\left(\xi_{a}^{\prime n}, \xi_{b}^{\prime n}\right)-\left(\xi_{a}^{n}, \xi_{b}^{n}\right)|=|\left(\xi_{a}^{\prime n}, \xi_{b}^{\prime n}\right)-\left(\xi_{a}^{n}, \xi_{b}^{n}\right)+\left(\xi_{a}^{\prime n}, \xi_{b}^{n}\right)-\left(\xi_{a}^{\prime n}, \xi_{b}^{n}\right) \mid \\
& \leq\left\|\xi_{a}^{\prime n}\right\|\left\|\xi_{b}^{\prime n}-\xi_{b}^{n}\right\|+\left\|\xi_{b}^{n}\right\|\left\|\xi_{a}^{n}-\xi_{a}^{\prime n}\right\| \rightarrow 0 .
\end{aligned}
$$

Onde foi usado a desigualdade de Cauchy-Schwarz. Então, $\lim \left(\xi_{a}^{\prime n}, \xi_{b}^{\prime n}\right)=$ $\lim \left(\xi_{a}^{n}, \xi_{b}^{n}\right)$. Para mostrar que o limite de (6) existe basta fazer uma conta similar a conta acima, mas desenvolvendo $\left|\left(\xi_{a}^{n}, \xi_{b}^{n}\right)-\left(\xi_{a}^{m}, \xi_{b}^{m}\right)\right|$, mostrando que $\left\{\left(\xi_{a}^{n}, \xi_{b}^{n}\right)\right\}_{n \in \mathbb{N}} \subset \mathbb{C}$ é uma sequência de Cauchy em $\mathbb{C}$ e, portanto, converge. Que (6) define um produto interno em $\mathscr{H}_{\psi}$ é elementar ${ }^{20}$.

\footnotetext{
${ }^{20}$ Em alguns espaços pre-Hilbert pode haver o caso em que $\|x\|=0$ para $x \neq 0$, e nesse caso toma-se como o espaço de Hilbert o conjunto $\mathscr{H} / N$, onde $N=\{x \in \mathscr{H}:(x, x)=0\}$, no entanto, no presente caso não é preciso fazer isso, pois $L_{\psi}$ é fechado (e isso se verifica facilmente tendo em mente a continuidade de $\psi$ ), então, se $\lim \xi_{a}^{n}=0 \in L_{\psi}$ significa que $\left\{\xi_{a}^{n}\right\} \subset L_{\psi}$, ou seja, é a sequência formada por elementos neutros de $D_{\psi}$.
} 
Basta agora mostrar que $\mathscr{H}_{\psi}$ é completo. Sejam sequências de Cauchy em $\mathscr{H}_{\psi}, \xi_{k}=\left\{\xi_{n}^{k}\right\}_{n}$ tal que para $n_{k}>0$ se tenha

$$
\left\|\xi_{m}^{k}-\xi_{n_{k}}^{k}\right\|<\frac{1}{k}, m>n_{k} .
$$

Isso pode ser sempre feito pois $\left\{\xi_{n}^{k}\right\}$ é de Cauchy.

Para mostrar que $\mathscr{H}_{\psi}$ é completo temos que mostrar que $\left\{\xi_{n}^{k}\right\}$ converge à um elemento de $\mathscr{H}_{\psi}$, digamos, $\left[\left\{\xi_{n_{1}}^{1}, \xi_{n_{2}}^{2}, \ldots, \xi_{n_{k}}^{k}, \ldots\right\}\right]$ (nota: $[X]$ sempre denota a classe de equivalência que contém $X$ ). Para isso, sejam

$$
\mathscr{H}_{\psi} \ni \bar{\xi}_{n_{k}}^{k}=\left[\left\{\xi_{n_{k}}^{k}, \xi_{n_{k}}^{k}, \ldots, \xi_{n_{k}}^{k}, \ldots\right\}\right], \quad k=1,2, \ldots
$$

Então, sabendo ainda que $\left\|\xi_{k}-\bar{\xi}_{n_{k}}^{k}\right\|=\lim _{m \rightarrow \infty}\left\|\xi_{m}^{k}-\xi_{n_{k}}^{k}\right\| \leq 1 / k$ e que $\left\|\bar{\xi}_{n_{k}}^{k}\right\|=\left\|\xi_{n_{k}}^{k}\right\|$ :

$$
\begin{aligned}
& \left\|\xi_{n_{k}}^{k}-\xi_{n_{m}}^{m}\right\|=\left\|\bar{\xi}_{n_{k}}^{k}-\bar{\xi}_{n_{m}}^{m}\right\|=\left\|\bar{\xi}_{n_{k}}^{k}+\xi_{k}-\xi_{k}-\bar{\xi}_{n_{m}}^{m}+\xi_{m}-\xi_{m}\right\| \\
& \leq\left\|\bar{\xi}_{n_{k}}^{k}-\xi_{k}\right\|+\left\|\bar{\xi}_{n_{m}}^{m}-\xi_{m}\right\|+\left\|\xi_{k}-\xi_{m}\right\| \leq \frac{1}{k}+\frac{1}{m}+\left\|\xi_{k}-\xi_{m}\right\| .
\end{aligned}
$$

Portanto, $\left\{\xi_{n_{1}}^{1}, \ldots, \xi_{n_{k}}^{k}, \ldots\right\} \subset D_{\psi}$ é uma sequência de Cauchy. Seja

$$
\mathscr{H}_{\psi} \ni \xi=\left[\left\{\xi_{n_{1}}^{1}, \ldots, \xi_{n_{k}}^{k}, \ldots\right\}\right] .
$$

Por fim, devemos mostrar que $\mathscr{H}_{\psi} \ni \xi_{k} \rightarrow \xi \in \mathscr{H}_{\psi}$, completando a prova de que $\mathscr{H}_{\psi}$ é completo. Então:

$$
\left\|\xi-\xi_{k}\right\| \leq\left\|\xi-\bar{\xi}_{n_{k}}^{k}\right\|+\left\|\bar{\xi}_{n_{l}}^{k}-\xi_{k}\right\| \leq\left\|\xi-\bar{\xi}_{n_{k}}^{k}\right\|+\frac{1}{k} .
$$

Mas,

$$
\begin{gathered}
\left\|\xi-\bar{\xi}_{n_{k}}^{k}\right\|=\lim _{p \rightarrow \infty}\left\|\xi_{n_{p}}^{p}-\xi_{n_{k}}^{k}\right\|=\lim _{p \rightarrow \infty}\left\|\xi_{n_{p}}^{p}-\xi_{p}+\xi_{p}+\xi_{k}-\xi_{k}+\xi_{n_{k}}^{k}\right\| \\
\leq \lim _{p \rightarrow \infty} \frac{1}{p}+\frac{1}{k}+\lim _{p \rightarrow \infty}\left\|\xi_{p}-\xi_{k}\right\| .
\end{gathered}
$$

Portanto, $\lim _{k \rightarrow \infty}\left\|\xi-\xi_{n_{k}}^{k}\right\|=0$. Então, $\lim _{k \rightarrow \infty}\left\|\xi_{k}-\xi\right\|=0$.

Além disso podemos construir uma isometria e isomorfismo entre $D_{\psi}$ e $\mathscr{H}_{\psi}$, de tal forma que podemos falar da densidade de $D_{\psi}$ em $\mathscr{H}_{\psi}$ (e há aqui e doravante um abuso de linguagem). Para isso considero a aplicação $D_{\psi} \ni$ $\xi_{a} \mapsto\left[\left\{\xi_{a}, \xi_{a}, \ldots, \xi_{a}, \ldots\right\}\right] \in \mathscr{H}_{\psi}$. Claramente isso é uma isometria e um isomorfismo, e seja $D_{\psi} \subset \mathscr{H}_{\psi}$ o conjunto formado por tais classes de equivalência de sequências constantes, vou mostrar que $D_{\psi}$ é denso em $\mathscr{H}_{\psi}$. 
Seja $\xi=\left[\left\{\xi^{1}, \xi^{2}, \ldots\right\}\right] \in \mathscr{H}_{\psi}$ uma classe de equivalência de sequências de Cauchy $\left\{\xi^{n}\right\}_{n}$. Seja $\left\{\xi_{k}\right\}=\left\{\left[\left\{\xi^{k}, \xi^{k}, \ldots, \xi^{k}, \ldots\right\}\right]\right\} \subset D_{\psi} \subset \mathscr{H}_{\psi}$, então, $\left\|\xi-\xi_{k}\right\|=\lim _{n \rightarrow \infty}\left\|\xi^{n}-\xi_{k}\right\| \leq \varepsilon$, já que $\left\{\xi^{n}\right\}_{n}$ é de Cauchy.

(ii) Agora definimos a representação $\pi_{\psi}$ de $\mathscr{A}$ em $\mathscr{H}_{\psi}$, e para isso devemos definir uma representação $\pi_{\psi}$ de $\mathscr{A}$ em $D_{\psi}$, que é denso em $\mathscr{H}_{\psi}$, e mostrar que é linear e continuo, de tal forma que podemos estendê-la à um operador linear e continuo em $\mathscr{H}_{\psi}$.

Seja $\pi_{\psi}: \mathscr{A} \rightarrow B\left(D_{\psi}\right)$ dada por:

$$
\pi_{\psi}(a) \xi_{b}=\xi_{a b}, \forall a, b \in \mathscr{A}
$$

Essa é uma boa definição, pois não depende do representante $b$ da classe $\xi_{b}$. De fato, seja $b^{\prime}=b+l, l \in L_{\psi}$

$$
\pi_{\psi}(a) \xi_{b^{\prime}}=\pi_{\psi}(a) \xi_{b+l}=\xi_{a b+a l} .
$$

Como al $\in L_{\psi}$, $\xi_{a l}$ é o elemento neutro de $D_{\psi}$. Portanto, $\pi_{\psi}(a) \xi_{b^{\prime}}=\xi_{a b}=$ $\pi_{\psi}(a) \xi_{b}$. Além disso $\pi_{\psi}(a)$ é linear:

$$
\pi_{\psi}(a)\left(\alpha \xi_{b}+\xi_{c}\right)=\pi_{\psi}(a) \xi_{\alpha b+c}=\xi_{\alpha a b+c}=\alpha \pi_{\psi}(a) \xi_{b}+\pi_{\psi}(a) \xi_{c} .
$$

E por fim $\pi_{\psi}(a)$ é limitado (e, portanto, continuo):

$$
\left\|\pi_{\psi}(a) \xi_{b}\right\|^{2}=\left(\xi_{a b}, \xi_{a b}\right)=\psi\left(b^{*} a^{*} a b\right) \leq\|a\|^{2} \psi\left(b^{*} b\right)=\|a\|^{2}\|b\|^{2} .
$$

Basta mostrar agora que $\pi_{\psi}(a)$ é um *-homomorfismo. Seja $\xi_{b} \in D_{\psi}$ e $a_{1}, a_{2} \in \mathscr{A}:$

$$
\pi_{\psi}\left(a_{1}\right) \pi_{\psi}\left(a_{2}\right) \xi_{b}=\pi_{\psi}\left(a_{1}\right) \xi_{a_{2} b}=\xi_{a_{1} a_{2} b}=\pi_{\psi}\left(a_{1} a_{2}\right) \xi_{b}
$$

Portanto, $\pi_{\psi}\left(a_{1}\right) \pi_{\psi}\left(a_{2}\right)=\pi_{\psi}\left(a_{1} a_{2}\right)$. E também, para $\xi_{a}, \xi_{b}, \xi_{c} \in D_{\psi}$ :

$$
\left(\pi_{\psi}(a) \xi_{b}, \xi_{c}\right)=\left(\xi_{a b}, \xi_{c}\right)=\psi\left((a b)^{*} c\right)=\psi\left(b^{*}\left(a^{*} c\right)\right)=\left(\xi_{b}, \xi_{a^{*} c}\right)=\left(\xi_{b}, \pi_{\psi}\left(a^{*}\right) \xi_{c}\right) .
$$

E como $D_{\psi}$ é denso em $\mathscr{H}_{\psi}, \pi_{\psi}\left(a^{*}\right)=\left(\pi_{\psi}(a)\right)^{*}$.

(iii) Nesta terceira parte definimos o vetor $\Omega_{\psi}$ de $\mathscr{H}_{\psi}$, completando a construção do terno desejado. Basta apenas tomar:

$$
\Omega_{\psi}=\xi_{\mathbb{1}}
$$

E, de fato:

$$
\left(\Omega_{\psi}, \pi_{\psi}(a) \Omega_{\psi}\right)=\left(\xi_{\mathbb{1}}, \xi_{a}\right)=\psi\left(\mathbb{1}^{*} a\right)=\psi(a) .
$$


E ainda:

$$
\pi_{\psi}(\mathscr{A}) \Omega_{\psi}=\left\{\pi_{\psi}(a) \Omega_{\psi}: a \in \mathscr{A}\right\}=\left\{\xi_{a}: a \in \mathscr{A}\right\}=D_{\psi}
$$

que é denso em $\mathscr{H}_{\psi}$.

(iv) Finalmente, a unicidade (à não ser por equivalência unitária) desses objetos. Suponha que hajam outros $\left(\mathscr{H}_{\psi}^{\prime}, \pi_{\psi}^{\prime}, \Omega_{\psi}^{\prime}\right)$ que satisfaçam as propriedades desejadas. Tome $U: D_{\psi} \rightarrow D_{\psi}^{\prime}$ como:

$$
U \pi_{\psi}(a) \Omega_{\psi}=\pi_{\psi}^{\prime} \Omega_{\psi}^{\prime}
$$

Então, $U$ é linear, pois:

$$
\begin{gathered}
U\left(\alpha \pi_{\psi}\left(a_{1}\right) \Omega_{\psi}+\pi_{\psi}\left(a_{2}\right) \Omega_{\psi}\right)=U\left(\pi_{\psi}\left(\alpha a_{1}+a_{2}\right) \Omega_{\psi}\right) \\
=\pi_{\psi}^{\prime}\left(\alpha a_{1}+a_{2}\right) \Omega_{\psi}^{\prime}=\alpha \pi_{\psi}^{\prime}\left(a_{1}\right) \Omega_{\psi}^{\prime}+\pi_{\psi}^{\prime}\left(a_{2}\right) \Omega_{\psi}^{\prime} .
\end{gathered}
$$

E ainda:

$$
\begin{gathered}
\left(U \pi_{\psi}\left(a_{1}\right) \Omega_{\psi}, U \pi_{\psi}\left(a_{2}\right) \Omega_{\psi}\right)=\left(\pi_{\psi}^{\prime}\left(a_{1}\right) \Omega_{\psi}^{\prime}, \pi_{\psi}^{\prime}\left(a_{2}\right) \Omega_{\psi}^{\prime}\right) \\
=\left(\xi_{a_{1}}^{\prime}, \xi_{a_{2}}^{\prime}\right)=\psi\left(a_{1}^{*} a_{2}\right)=\left(\pi_{\psi}\left(a_{1}\right) \Omega_{\psi}, \pi_{\psi}\left(a_{2}\right) \Omega_{\psi}\right) .
\end{gathered}
$$

Ou seja, $U$ é uma isometria linear do subespaço $\pi_{\psi}(\mathscr{A}) \Omega_{\psi}$ no subespaço $\pi_{\psi}^{\prime}(\mathscr{A}) \Omega_{\psi}^{\prime}$, ou seja, seu fecho (chamado também de $U$ ) é um operador unitário de $\mathscr{H}_{\psi}$ em $\mathscr{H}_{\psi}^{\prime}$. E ainda:

$$
\begin{gathered}
U \pi_{\psi}\left(a_{1}\right) \pi_{\psi}\left(a_{2}\right) \Omega_{\psi}=U \pi_{\psi}\left(a_{1} a_{2}\right) \Omega_{\psi}=\pi_{\psi}^{\prime}\left(a_{1} a_{2}\right) \Omega_{\psi}^{\prime} \\
=\pi_{\psi}^{\prime}\left(a_{1}\right)\left(\pi_{\psi}^{\prime}\left(a_{2}\right) \Omega_{\psi}^{\prime}\right)=\pi_{\psi}^{\prime}\left(a_{1}\right) U \pi_{\psi}\left(a_{2}\right) \Omega_{\psi} .
\end{gathered}
$$

Ou seja,

$$
U \pi_{\psi}(a)=\pi_{\psi}^{\prime}(a) U
$$

E também:

$$
U \Omega_{\psi}=U \pi_{\psi}(\mathbb{1}) \Omega_{\psi}=\psi_{\pi}^{\prime}(\mathbb{1}) \Omega_{\psi}^{\prime}=\Omega_{\psi}^{\prime} .
$$

Isso completa a prova do teorema.

\subsection{Propriedades das CAR e CCR e suas álgebras}

Nessa seção analisaremos os operadores de aniquilação e criação sem que eles estejam necessariamente num espaço de Fock, descobrindo algumas de suas propriedades. E além disso abstrairemos desses operadores - já definidos em espaços de Hilbert não necessariamente de Fock - suas essências algébricas: definiremos álgebras cujas representações fiéis são os operadores 
que agem em um espaço de Hilbert, isto é, tomaremos as relações canônicas de (anti)comutação como uma propriedade essencial de uma álgebra e veremos algumas das suas propriedades.

Aqui os $a_{-}(f)$ de $a_{+}(f)$ serão separados mais nitidamente, de modo que em cada subseção omitirei os índices. É preciso fazer essa separação pois, como veremos, os operadores $a_{+}(f)$ e $a_{+}^{*}(f)$ não são limitados, enquanto seus homólogos antissimétricos são limitados. Para evitar os problemas que operadores não limitados trazem construiremos operador $\Phi(f)$ auto-adjunto, a partir do qual $a_{+}(f)$ e $a_{+}^{*}(f)$ podem ser recuperados, e considerar uma função de $\Phi(f)$ que fornecem operadores limitados, a partir dos quais obtemos as relações canônicas de comutação em outra forma, a saber, a forma de Weyl.

\subsubsection{CAR e suas álgebras}

Uma $C^{*}$-álgebra CAR é uma $C^{*}$-álgebra $\mathscr{A}(\mathscr{H})$ gerada por $\mathbb{1}$ e $a(f), f \in$ $\mathscr{H}, \mathscr{H}$ é um espaço de Hilbert, tal que: $(i) f \mapsto a(f)$ é antilinear; $(i i)$ $\{a(f), a(g)\}=0, \forall f, g \in \mathscr{H}$ e $(i i i)\left\{a(f), a^{*}(g)\right\}=(f, g) \mathbb{1}$.

Propriedades (de [10]):

(1) Essa álgebra é única a não ser por um *-isomorfismo (i.e., se há duas álgebras $\mathscr{A}(\mathscr{H})_{1}$ e $\mathscr{A}(\mathscr{H})_{2} \mathrm{CAR}$, então existe um único ${ }^{*}$-isomorfismo $\alpha$ : $\mathscr{A}(\mathscr{H})_{1} \rightarrow \mathscr{A}(\mathscr{H})_{2}$, tal que $\alpha\left(a_{1}(f)\right)=a_{2}(f)$.)

(2) $\|a(f)\|=\|f\|, \forall f \in \mathscr{H}$

(3) $\mathscr{A}(\mathscr{H})$ é separável $\Leftrightarrow \mathscr{H}$ é separável;

(4) $\mathscr{A}(\mathscr{H})$ é simples;

(5) Se $U$ e $V$ são operadores limitados em $\mathscr{H}$ e $U$ linear e $V$ antilinear, tais que

$$
\begin{aligned}
& V^{*} U+U^{*} V=U V^{*}+V U^{*}=0, \\
& U^{*} U+V^{*} V=U U^{*}+V V^{*}=\mathbb{1},
\end{aligned}
$$

Então, existe um único ${ }^{*}$-automorfismo $\gamma$ em $\mathscr{A}(\mathscr{H})$ tal que:

$$
\begin{gathered}
\gamma(a(f))=a(U f)+a^{*}(V f), \\
\gamma^{-1}(a(f))=a\left(U^{*} f\right)+a^{*}\left(V^{*} f\right) .
\end{gathered}
$$

Provaremos apenas as propriedades (2) e (3): Para provar (2) basta multiplicar $a(f) a^{*}(f)+a^{*}(f) a(f)=\|f\|^{2}$ por $a^{*}(f) a(f)$. O primeiro termo é zero, pois $a(f) a(f)=0$, então,

$$
a^{*}(f) a(f) a^{*}(f) a(f)=a^{*}(f) a(f)\|f\|^{2} .
$$


Tomando a norma disso, e lembrando da propriedade das $\mathrm{C}^{*}$-álgebras:

$$
\left\|a^{*}(f) a(f)\right\|=\|a(f)\|^{2},
$$

obtemos $\|a(f)\|^{4}=\|f\|^{2}\|a(f)\|^{2} \therefore\|a(f)\|=\|f\|$.

Para o item (3): se $\mathscr{H}$ é separável há um conjunto $\left\{f_{n}\right\}_{n \in I}, I$ contável, que é denso em $\mathscr{H}$, ou seja, todo elemento de $\mathscr{H}$ é aproximado por combinações de $f_{n}$, e como $a(f)$ é antilinear (de tal forma que posso fazer $\left.a\left(f_{n}\right)-a(f)=a\left(f_{n}-f\right)\right)$ e $\|a(f)\|=\|f\|, a\left(f_{n}\right)$ aproxima todo elemento $a(f)$ de $\mathscr{A}(\mathscr{H})$, i.e., $\mathscr{A}(\mathscr{H})$ tem um conjunto contável $\left\{a\left(f_{n}\right)\right\}_{n \in I}$ que é denso em $\mathscr{A}(\mathscr{H})$. A volta é análoga.

Teorema 2.2 (Estrutura quasi-local da álgebra CAR, [10]). Seja $\mathscr{A}(\mathscr{H})$ a $C^{*}$-álgebra $C A R$ sobre $\mathscr{H}$, um espaço de Hilbert separável. Seja $\mathcal{I}$ uma rede de subespaços não vazios de $\mathscr{H}$, ordenados por inclusão, tal que:

(1) $M \in \mathcal{I} \Rightarrow \exists N \in \mathcal{I}$ tal que $M \perp N$;

(2) $M \perp N$ e $M \perp K \Rightarrow \exists L \in \mathcal{I}$ tal que $M \perp L$ e $N, K \subseteq L$;

(3) $\mathscr{H}=\overline{\bigcup_{M \in \mathcal{I}} M}$.

Seja também $\mathscr{A}(M) \subseteq \mathscr{A}(\mathscr{H})$ uma sub $C^{*}$-álgebra gerada por $\{a(f): f \in$ $M\}, M \in \mathcal{I}$. Então, $\left(\mathscr{A}(\mathscr{H}),\{\mathscr{A}(M)\}_{M \in \mathcal{I}}\right)$ é uma álgebra quasi-local com $\sigma(a(f))=-a(f)$.

Não provaremos este teorema (a prova pode ser encontrada em [10]) já que as álgebras CAR não serão o foco deste trabalho. Ao invés, provaremos o análogo deste teorema para o caso das CCR.

\subsubsection{CCR, a forma de Weyl e suas álgebras}

Novamente, afirmamos que os operadores que satisfazem a relação canônica de comutação não são ambos limitados: de fato, suponha que $p$ e $q$ sejam operadores limitados não nulos tais que $[p, q]=k \mathbb{1}, k>0$. Então:

$$
\begin{gathered}
\forall n \geq 1, \quad\left[p, q^{n}\right]=[p, q] q^{n-1}+q\left[p, q^{n-1}\right]=k q^{n-1}+q\left([p, q] q^{n-2}+q\left[p, q^{n-2}\right]\right) \\
=2 k q^{n-1}+q^{2}\left[p, q^{n-2}\right]=\ldots=n k q^{n-1} .
\end{gathered}
$$

Tomando a norma dessa igualdade (lembrando que assumimos, por absurdo, que $p$ e $q$ são limitados):

$$
k n\left\|q^{n-1}\right\|=\left\|p q^{n}-q^{n} q\right\| \leq\|p\|\left\|q^{n}\right\|+\left\|q^{n}\right\|\|p\|=2\|p\|\left\|q^{n} \cdot\right\|
$$

\footnotetext{
${ }^{21}$ Esse é o $\sigma$ da observação da seção anterior, que divide elementos da álgebra entre pares e impares
} 
Portanto, $\|p\|\|q\| \geq n k / 2, \quad \forall n \geq 1$, mostrando que esses operadores não podem ambos ser limitados.

Para evitar tratar com operadores não limitados fazemos a seguinte construção: consideramos os operadores de quantização de Segal:

$$
\Phi(f)=\frac{1}{\sqrt{2}}\left(a(f)+a^{*}(f)\right) .
$$

A partir dele recuperamos os operadores de criação e aniquilação, pois $\Phi(i f)=$ $\left(a(f)-a^{*}(f)\right) / i \sqrt{2}$.

O operador $\Phi(f)$ é essencialmente auto-adjunto em $D_{0}$, isso é provado usando o teorema de vetores analíticos de Nelson (que diz que para um operador simétrico $A$ em $\mathscr{H}$, se $D_{A}$ contém um conjunto total ${ }^{22}$ de vetores analítico ${ }^{23}$, então $A$ é essencialmente auto-ajunto). A prova em detalhes é encontrada em [26]

Além disso $\Phi(f)$ é continuo: $\left\|f_{\alpha}-f\right\| \rightarrow 0 \Rightarrow\left\|\left(\Phi\left(f_{\alpha}\right)-\Phi(f)\right) \psi\right\| \rightarrow 0, \forall \psi \in$ $D_{0}$. Prova:

$$
\begin{gathered}
\left\|\left(\Phi\left(f_{\alpha}\right)-\Phi(f)\right) \psi\right\|=\frac{1}{\sqrt{2}}\left\|\left(a\left(f_{\alpha}\right)+a^{*}\left(f_{\alpha}\right)-a(f)-a^{*}(f)\right) \psi\right\|= \\
=\frac{1}{2}\left\|a\left(f_{\alpha}-f\right) \psi+a^{*}\left(f_{\alpha}-f\right) \psi\right\| \\
\leq \frac{1}{\sqrt{2}}\left(\left\|a\left(f_{\alpha}-f\right) \psi\right\|+\left\|a^{*}\left(f_{\alpha}-f\right) \psi\right\|\right) \leq \frac{2}{\sqrt{2}}\left\|f_{\alpha}-f\right\|\|\sqrt{N+1} \psi\| \rightarrow 0 .
\end{gathered}
$$

Onde na última passagem usamos as relações demonstradas na seção (2.1.1), $\|a(f) \psi\| \leq n^{1 / 2}\|f\|\|\psi\|$ e $\left\|a^{*}(f) \psi\right\| \leq(n+1)^{1 / 2}\|f\|\|\psi\|$.

A relação de comutação é $[\Phi(f), \Phi(g)]=i \Im(f, g) \mathbb{1}$, onde $\Im z$ é a parte imaginaria de $z$. Prova:

$$
\begin{gathered}
{[\Phi(f), \Phi(g)] \psi=\frac{1}{2}\left(a(f)+a^{*}(f)\right)\left(a(g)+a^{*}(g)\right)-\frac{1}{2}\left(a(g)+a^{*}(g)\right)\left(a(f)+a^{*}(f)\right)} \\
=\frac{1}{2}\left[a(f) a^{*}(g)-a^{*}(g) a(f)+a^{*}(f) a(g)-a(g) a^{*}(f)\right]=\frac{1}{2}[(f, g)-(g, f)] \\
=\frac{1}{2}[(f, g)-\overline{(f, g)}]=i \Im(f, g) .
\end{gathered}
$$

\footnotetext{
${ }^{22}$ Um subconjunto $S$ de $\mathscr{H}$ é total se o conjunto de combinações lineares de elementos de $S$ for denso em $\mathscr{H}$.

${ }^{23} \mathrm{Um}$ vetor analítico de $A$ é um vetor $\phi \in C^{\infty}(A):=\bigcap_{n=1}^{\infty} D_{A^{n}}$ (i.e., ele está bem definido para todas as potências de $A$ ) tal que para algum $t>0$ valha $\sum_{n=0}^{\infty}\left\|A^{n} \phi\right\| t^{n} / n !<$ $\infty$.
} 
Chamamos o fecho de $\Phi(f)$ também de $\Phi(f)$, e agora ele é um operador auto-ajunto em $D_{0}$ e continuo. Pelo teorema espectral podemos definir os operadores de Weyl: $W(f)=e^{i \Phi(f)}$, que são operadores unitários. São as álgebras desses operadores que serão consideradas, e eles possuem a seguinte relação de comutação:

$$
W(f) W(g)=e^{-i \Im(f, g) / 2} W(f+g) .
$$

Álgebras de Weyl Passemos agora para uma definição da álgebra de Weyl de uma forma abstrata, sem assumir a priori que essa álgebra é formada por operadores agindo em um espaço de Hilbert.

A $C^{*}$-algebra de Weyl $\mathscr{A}(H)$ é a álgebra gerada por $W(f), f \in H$, onde $H$ é um espaço vetorial real com uma forma simplética $o{ }^{24}$ que satisfaz: $\forall f, g \in H$

$$
W(f)^{*}=W(-f), \quad W(f) W(g)=e^{-i \sigma(f, g) / 2} W(f+g) .
$$

Note que o caso anterior é recuperado quando $H$ é um espaço de Hilbert e $\sigma(f, g)=\Im(f, g)$.

Essa álgebra é única à não ser por um ${ }^{*}$-isomorfismo: se $\mathscr{A}_{1}$ e $\mathscr{A}_{2}$ são duas álgebras de Weyl, então existe um único ${ }^{*}$-isomorfismo $\alpha: \mathscr{A}_{1} \rightarrow \mathscr{A}_{2}$ tal que $\alpha\left(W_{1}(f)\right)=W_{2}(f)$. A prova desta afirmação se encontra em [10]. Além disso os elementos de $\mathscr{A}(H)$ são unitários e $W(0)=\mathbb{1}$, pois:

$$
\begin{gathered}
W(f) W(0)=W(f)=W(0) W(f) \Rightarrow W(0)=\mathbb{1}, \\
W(f) W(-f)=e^{-i \sigma(f,-f) / 2} W(0)=\mathbb{1}=W(-f) W(f),
\end{gathered}
$$

pois $\sigma(f,-f)=-\sigma(f, f)$ e $\sigma(f,-f)=-\sigma(-f, f)=\sigma(f, f)$, então, $\sigma(f,-f)=$ 0 e $W(-f)=W(f)^{*}$.

Teorema 2.3 (Estrutura quasi-local da álgebra de Weyl, [10]). Seja H um espaço vetorial com uma forma simplética não degenerada bilinear $\sigma$. Seja $\mathcal{I}$ o conjunto, ordenado por inclusão, formado por subespaços não vazios de $H$, com a relação $N \perp M \Leftrightarrow \sigma(f, g)=0, \forall f \in N, \forall g \in M$, tal que valham:

(i) $M \in \mathcal{I} \Rightarrow \exists N \in \mathcal{I}$ tal que $M \perp N$;

(ii) $M \perp N$ e $M \perp K \Rightarrow \exists L \in \mathcal{I}$ tal que $M \perp L$ e $N, K \subseteq L$;

(iii) $H=\bigcup_{M \in \mathcal{I}} M$.

Seja $\mathscr{A}(H)$ a $C^{*}$-álgebra de Weyl sobre $H$ e $\mathscr{A}_{M}$ a sub $C^{*}$-álgebra gerada

\footnotetext{
${ }^{24} \mathrm{Ou}$ seja, $\sigma: H \times H \rightarrow \mathbb{R}$ tal que: $\sigma(f, g)=-\sigma(g, f), \forall f, g \in H$ e $\sigma(f, g)=0, \forall f \in$ $H \Rightarrow g=0$
} 
por $\{W(f): f \in M\}, M \in \mathcal{I}$. Então, $\left(\mathscr{A}(H),\left\{\mathscr{A}_{M}\right\}_{M \in \mathcal{I}}\right)$ é uma álgebra quasi-local. Ou seja, valem as seguintes propriedades:

(1) $M \subseteq N \Rightarrow \mathscr{A}_{M} \subseteq \mathscr{A}_{N}$;

(2) $\mathscr{A}_{M}$ possui a identidade, para todo $M$;

(3) $M \perp N \Leftrightarrow\left[\mathscr{A}_{M}, \mathscr{A}_{N}\right]=0$;

(4) $\mathscr{A}(H)=\bigcup_{M} \mathscr{A}_{M}$.

Demonstração. Para o item (1): $M \subseteq N \Rightarrow\{W(f): f \in M\} \subseteq\{W(f): f \in$ $N\}$. Então, $\mathscr{A}_{M} \subseteq \mathscr{A}_{N}$.

Item (2): todo subespaço de $H$ contém $f=0$, então toda álgebra $\mathscr{A}_{M}$ contém $W(0)$.

Para o item (3) calculamos:

$$
W(f) W(g)=e^{-i \sigma(f, g) / 2} W(f+g), \quad W(g) W(f)=e^{i \sigma(f, g) / 2} W(f+g) .
$$

Portanto, $W(f) W(g)=e^{-i \sigma(f, g)} W(g) W(f)$, e se $M \perp N$ então, $f \in M$ e $g \in N$ implica em $\sigma(f, g)=0 \therefore W(f) W(g)=W(g) W(f)$.

Por fim, para o item (4): $\mathscr{A}(H)$ é gerado por $W(f), f \in H$, mas $H=\bigcup_{i} M_{i}$. Portanto, $\mathscr{A}(H)=\bigcup_{i} \mathscr{A}_{M_{i}}$.

Esse teorema nos garante que a álgebra CCR-Weyl tem a estrutura necessária para satisfazer os axiomas de Haag-Kastler, restando apenas a especificação do conteúdo relativístico. Este conteúdo - como também provado no teorema - não está completamente contido na especificação dos conjuntos de $H$ e da relação de ortogonalidade, mas de uma forma mais importante na forma simplética $\sigma$-mais importante pois nela estão codificadas as relações causais. 
3 O Conteúdo Relativístico 


\subsection{Revisão dos Conceitos Básicos}

Nesta seção revisaremos os conceitos básicos de geometria relevantes à teoria da relatividade, tanto por completeza como para fixação da notação que poderá ser eventualmente utilizada. O texto desta seção se restringirá aos aspectos mais superficiais da geometria, ou seja, os aspectos que serão utilizados mais à frente, sem se ter a pretensão de se elaborar nas definições ou investigar os detalhes mais intricados da teoria (poderia tomar as palavras do satirista romano Persius: minimum est quod scire laboro). De modo que esta seção certamente não tem como objetivo suprir alguma falta no conhecimento de geometria e relatividade.

O principal texto utilizado nas definições é O'neill [25], mas também [20], [34, [36] e capítulo 33 de [4]. A seção sobre distrubições em variedades foi inspirada principalmente por Hörmander, [22], e também por [16]. Na seção sobre estruturas causais utilizamos [2], [3], [11], [25] e [6], [7], [8].

Uma variedade infinitamente diferenciável de dimensão $n$ é um espaço topológico cujos abertos são homeomorfos à abertos em $\mathbb{R}^{n}$, que admite um cobrimento contável por atlas $\left\{\left(\mathscr{U}_{i}, h_{i}\right)\right\}$, com uma estrutura diferenciável (i.e., dotado da classe de equivalência entre atlas - e dois atlas são equivalentes se a união for um atlas infinitamente diferenciável). Denotaremos variedades diferenciáveis que são Hausdorff e segundo-contaveis por $\mathscr{M}$.

Sejam a função $f: \mathscr{M} \rightarrow \mathbb{R}$ e a carta local $(\mathscr{U}, h)$, as coordenadas de $f \mathrm{em}$ relação à esta carta é a função $f \circ h^{-1}: h(\mathscr{U}) \subset \mathbb{R}^{n} \rightarrow \mathbb{R}$. $f$ é dita ser suave se $f \circ h^{-1}$ for suave (i.e., se todas as suas derivadas existirem e forem continuas), para toda carta local de $\mathscr{M}$. O conjunto de todas as funções suaves em $\mathscr{M}$ é denotado por $\mathfrak{F}(\mathscr{M})$.

Dada duas variedades $\mathscr{M}$ e $\mathscr{N}$, com dimensões $m$ e $n$, respectivamente, a aplicação $\phi: \mathscr{M} \rightarrow \mathscr{N}$ é suave se para toda carta local de $\mathscr{M}$ e $\mathscr{N}$, digamos, $(\mathscr{U}, h)$ e $(\mathscr{V}, w)$ a função $w \circ \phi \circ h^{-1}: \mathbb{R}^{m} \rightarrow \mathbb{R}^{n}$ for suave. $\phi$ é um difeomorfismo se $\phi$ e $\phi^{-1}$ forem suaves.

Uma curva é a aplicação $\alpha: I \rightarrow \mathscr{M}$, onde $I$ é um intervalo nos reais.

Partições da Unidade Usamos partições da unidade para decompor objetos globais em objetos locais, e unir objetos locais em um objeto global. Este é, portanto, um conceito muito útil. Vejamos sua definição. Uma partição da unidade suave em $\mathscr{M}$ é o conjunto $\left\{f_{\alpha} \in \mathfrak{F}(\mathscr{M}): \alpha \in A\right\}$, onde $A$ é algum conjunto de índices, tal que: $0 \leq f_{\alpha} \leq 1, \forall \alpha \in A ;\left\{\operatorname{supp} f_{\alpha}: \alpha \in A\right\} \subset \mathscr{M}$ é localmente finito (i.e., há uma vizinhança de qualquer ponto de $\mathscr{M}$ que intersecta um número finito de pontos de $\left\{\operatorname{supp} f_{\alpha}\right\}$, isso significa que nessa vizinhança apenas um número finito de $f_{\alpha}$ 's serão não-nulos, e essa condição 
nos garante a possibilidade da próxima condição); $\sum_{\alpha} f_{\alpha}=1$.

Uma partição da unidade suave é subordinada ao cobrimento $\left\{\left(\mathscr{U}_{\alpha}, h_{\alpha}\right)\right\}$ de $\mathscr{M}$ se cada $\operatorname{supp} f_{\alpha}$ está contida em $\mathscr{U}_{\alpha}$. É possível provar que em toda variedade segundo contável há uma partição da unidade suave subordinada a todo cobrimento.

Vetores Tangentes Seja $p \in \mathscr{M}$ um ponto da variedade $\mathscr{M}$, um vetor tangente à $p$ é o funcional $v: \mathfrak{F}(\mathscr{M}) \rightarrow \mathbb{R}$, que satisfaz as seguintes duas propriedades:

$$
v(a f+b g)=a v(f)+b v(g), \quad v(f g)=v(f) g(p)+f(p) v(g), \quad \forall a, b \in \mathbb{R} .
$$

O conjunto $T_{p}(\mathscr{M})$ é o conjunto dos vetores tangentes a $p$, e $T_{p}(\mathscr{M})$ é um espaço vetorial, definindo soma e multiplicação por escalares da forma usual. Podemos construir uma base desse espaço vetorial, da seguinte forma: dada a carta $(\mathscr{U}, h)$ que contém $p$, e, digamos que $h$ estabeleça o sistema de coordenadas locais $h=\left(y^{1}, \ldots, y^{n}\right)$, então, se $f \in \mathfrak{F}(\mathscr{M})$ :

$$
\frac{\partial f}{\partial y^{i}}(p)=\frac{\partial\left(f \circ h^{-1}\right)}{\partial x^{i}}(h(p))
$$

onde $\left\{x^{i}\right\}$ é o sistema de coordenadas naturais do $\mathbb{R}^{n}$. É fácil de verificar que a aplicação

$$
\left.\partial_{i}\right|_{p}=\left.\frac{\partial}{\partial y^{i}}\right|_{p}: \mathfrak{F}(\mathscr{M}) \rightarrow \mathbb{R}
$$

é um vetor tangente em $p$, e a base de $T_{p}(\mathscr{M})$ é formada por $\left\{\left.\partial_{1}\right|_{p}, \ldots,\left.\partial_{n}\right|_{p}\right\}$. Decompomos, então, $v \in T_{p}(\mathscr{M})$ da seguinte forma: $v=\left.v^{i} \partial_{i}\right|_{p}$, onde $v^{i}=$ $v\left(y^{i}\right)$ e a soma nos índices repetidos está implícita.

Um campo vetorial em $\mathscr{M}$ é a associação $V$ entre cada ponto de $\mathscr{M}$ à um vetor tangente à si. $V$ é suave se, $\forall f \in \mathfrak{F}(\mathscr{M}), V() f:. \mathscr{M} \rightarrow \mathbb{R}$ for suave, i.e., $V f \in \mathfrak{F}(\mathscr{M})$. O conjunto dos campos vetoriais suaves em $\mathscr{M}$ é denotado pelo simbolo $\mathfrak{X}(\mathscr{M})$. $\mathfrak{X}(\mathscr{M})$ é um módulo sobre o anel $\mathfrak{F}(\mathscr{M})^{25}$ se definirmos $(f V)_{p}=f(p) V_{p}$ e $(V+W)_{p}=V_{p}+W_{p}$. Numa carta local com coordenadas $\left\{x^{i}\right\}$, com o campo vetorial $\partial_{i}$ que faz a associação $\left.p \mapsto \partial_{i}\right|_{p}$ obtemos uma base de $\mathfrak{X}(\mathscr{M})$, e portanto escrevemos $V=V^{i} \partial_{i}$, onde $V^{i}=V x^{i}$, e claro, $\partial_{i} f=\partial f / \partial x^{i}$.

Com espaços de vetores tangentes à um ponto podemos aproximar aplicações suaves à aplicações lineares (entre espaços tangentes), e isso nos fornece o conceito de uma diferencial de uma aplicação. Notemos que dada a aplicação

\footnotetext{
${ }^{25}$ Formalmente é o mesmo que um espaço vetorial, mas com o anel $\mathfrak{F}(\mathscr{M})$ agindo como corpo.
} 
suave $\phi: \mathscr{M} \rightarrow \mathscr{N}$, podemos para cada $v \in T_{p}(\mathscr{M})$ associar um $v_{\phi} \in$ $T_{\phi(p)}(\mathscr{N})$, segundo a seguinte ação em $g \in \mathfrak{F}(\mathscr{N}): v_{\phi}(g)=v(g \circ \phi)$.

O diferencial da aplicação suave $\phi: \mathscr{M} \rightarrow \mathscr{N}$ é a função que associa, para todo $p, v$ à $v_{\phi}$, ou seja,

$$
\begin{gathered}
d \phi_{p}: T_{p}(\mathscr{M}) \rightarrow T_{\phi(p)}(\mathscr{N}), \\
\left(d \phi_{p}(v)\right)(g)=v(g \circ \phi), \quad \forall g \in \mathfrak{F}(\mathscr{N}) .
\end{gathered}
$$

A velocidade de uma curva $\alpha: I \mapsto \mathscr{M}$ em $t$ é um vetor $\alpha^{\prime}(t) \in T_{\alpha(t)}(\mathscr{M})$, dado por

$$
\alpha^{\prime}(t)=d \alpha\left(\left.\frac{d}{d x}\right|_{t}\right)
$$

Temos, então, $\left(\alpha^{\prime}(t)\right)(g)=\left(d \alpha\left(\left.\frac{d}{d x}\right|_{t}\right)\right)(g)=\left.\frac{d}{d x}\right|_{t}(g \circ \alpha)$. Portanto, sendo $\left\{x^{i}\right\}$ coordenadas em torno de $\alpha(t), \alpha^{\prime}(t)$ se escreve como

$$
\alpha^{\prime}(t)=\left.\alpha^{\prime}(t)^{i} \partial_{i}\right|_{\alpha(t)}=\left.\alpha^{\prime}(t)\left(x^{i}\right) \partial_{i}\right|_{\alpha(t)}=\left.\left.\frac{d}{d x}\left(x^{i} \circ \alpha\right)\right|_{t} \partial_{i}\right|_{\alpha(t)} .
$$

1-formas 1-formas em $\mathscr{M}$ são duais de campos vetoriais em $\mathscr{M}$. Ou seja, dado o espaço $T_{p}(\mathscr{M})$, temos o seu dual $T_{p}(\mathscr{M})^{*}$, chamado de espaço cotangente, e que consiste nos funcionais lineares de $T_{p}(\mathscr{M})$, e 1 -formas em $\mathscr{M}$ associam pontos de $\mathscr{M}$ nos duais de seus vetores tangentes, ou seja, é a aplicação $\theta: \mathscr{M} \rightarrow T_{p}(\mathscr{M})^{*}, \quad \forall p \in \mathscr{M}$.

$\theta$ é suave se, $\forall X \in \mathfrak{X}(\mathscr{M}), \theta() X:. \mathscr{M} \rightarrow \mathbb{R}$ for suave, e o conjunto de todas as 1 -formas suaves é $\mathfrak{X}(\mathscr{M})^{*}$, e é um módulo sobre $\mathfrak{F}(\mathscr{M})$, se definirmos soma e multiplicação por elementos de $\mathfrak{F}(\mathscr{M})$ da forma usual.

O exemplo canônico de 1 -formas é o diferencial df de $f \in \mathfrak{F}(\mathscr{M})$, caracterizado por $(d f)(v)=v(f), \forall v \in \mathfrak{X}(\mathscr{M})$. Dos diferenciais podemos construir elementos que geram o espaço das 1-formas, elementos análogos aos $\partial_{i}$ no caso de vetores. Seja a carta $\left(\mathscr{U}_{\alpha}, h_{\alpha}\right)$ com coordenadas $\left\{x^{i}\right\}$, temos os seus diferenciais $\left\{d x^{i}\right\}$, e claramente, $d x^{i}\left(\partial_{j}\right)=\partial_{j}\left(x^{i}\right)=\delta_{i j}$, e da mesma maneira que no caso de vetores decompomos uma 1-forma: $\theta=\theta_{i} d x^{i}$, onde $\theta_{i}=\theta\left(\partial_{i}\right)$

Tensores Tensores podem ser definidos em espaços vetoriais arbitrários: seja $V$ um espaço vetorial (ou em geral um módulo sobre um anel) sobre $\mathbb{K}$ e $V^{*}$ seu dual (i.e., o espaço das funções de $V$ em $\mathbb{K}$ lineares), um tensor do tipo $(r, s)$ é a função $\mathbb{K}$-multilinear (linear com respeito a $\mathbb{K}$ em todas suas "entradas") $A:\left(V^{*}\right)^{r} \times V^{s} \rightarrow \mathbb{K}$.

No presente contexto tomamos $\mathbb{K}=\mathfrak{F}(\mathscr{M})$ e $V=\mathfrak{X}(\mathscr{M})$, então $A$ do tipo $(r, s)$ é a função multilinear $A: \mathfrak{X}^{*}(\mathscr{M})^{r} \times \mathfrak{X}(\mathscr{M})^{s} \rightarrow \mathfrak{F}(\mathscr{M})$. O conjunto dos tensores do tipo $(r, s)$ em $\mathscr{M}$ é $\mathfrak{T}^{r, s}(\mathscr{M})$. 
Ou seja, dado um conjunto de $r$ 1-formas e $s$ vetores devemos ter uma função em $\mathfrak{F}(\mathscr{M})$ que depende $\mathfrak{F}(\mathscr{M})$-linearmente dessas 1-formas e vetores:

$$
A\left(\theta^{1}, \ldots, \theta^{r}, V_{1}, \ldots, V_{s}\right) \in \mathfrak{F}(\mathscr{M}) .
$$

E, por exemplo,

$$
A\left(\theta^{1}, \ldots, f \theta^{i}, \ldots, \theta^{r}, V_{1}, \ldots, g V_{i}, \ldots, V_{s}\right)=f g A\left(\theta^{1}, \ldots, \theta^{r}, V_{1}, \ldots, V_{s}\right) .
$$

Podemos interpretar $\mathfrak{T}^{0,1}(\mathscr{M})$ como o conjunto das 1-formas suaves em $\mathscr{M}$, e $\mathfrak{T}^{1,0}(\mathscr{M})$ como o $\mathfrak{X}(\mathscr{M})$ (essas identificações feitas através de isomorfismos, vide, e.g., [25]). Então, numa carta local $\partial_{i}$ e $d x^{i}$ seriam exemplos de tensores do tipo $(1,0)$ e $(0,1)$, respectivamente.

O produto tensorial é uma operação que de dois tensores, digamos $A \in$ $\mathfrak{T}^{r, s}(\mathscr{M})$ e $B \in \mathfrak{T}^{r^{\prime}, s^{\prime}}(\mathscr{M})$ obtemos um terceiro tensor $A \otimes B$ do tipo $(r+$ $\left.r^{\prime}, s+s^{\prime}\right)$, dado por:

$$
\begin{gathered}
(A \otimes B)\left(\theta^{1}, \ldots, \theta^{r+r^{\prime}}, V_{1}, \ldots, V_{s+s^{\prime}}\right) \\
=A\left(\theta^{1}, \ldots, \theta^{r}, V_{1}, \ldots, V_{s}\right) B\left(\theta^{r+1}, \ldots, \theta^{r+r^{\prime}}, V_{1+s}, \ldots, V_{s+s^{\prime}}\right) .
\end{gathered}
$$

Uma contração é uma aplicação em tensores que transforma um tensor do tipo $(r, s)$ num tensor do tipo $(r-1, s-1)$, definido da seguinte forma: há $([25])$ uma única aplicação $C: \mathfrak{T}^{1,1}(\mathscr{M}) \rightarrow \mathfrak{F}(\mathscr{M})$ dada por $C(V \otimes \theta)=$ $\theta V, \forall V \in \mathfrak{X}(\mathscr{M}), \forall \theta \in \mathfrak{X}(\mathscr{M})^{*}$. Estendemos essa aplicação para um tensor mais geral da seguinte forma: seja $A \in \mathfrak{T}^{r, s}(\mathscr{M})$, e sejam $r-11$-formas $\theta^{k} \mathrm{e}$ $s-1$ vetores $V_{k}$, então, $f(\theta, V)=A\left(\theta^{1}, \ldots, \theta, \ldots, \theta^{r-1}, V_{1}, \ldots, V, \ldots, V_{s-1}\right) \in$ $\mathfrak{T}^{1,1}(\mathscr{M})$, onde $\theta$ e $V$ estão nos "slots" $i$ e $j$, respectivamente. Aplicando $C$ em $f$ obtemos outra função, chamemos esta de $C_{j}^{i} A$, que dependerá $\mathfrak{F}(\mathscr{M})$ linearmente das $r-1$ 1-formas e dos $s-1$ vetores, sendo, portanto, um tensor em $\mathfrak{T}^{r-1, s-1}(\mathscr{M}){ }^{26}$

As componentes de um tensor $A \in \mathfrak{T}^{r, s}(\mathscr{M})$ são fornecidas no calculo de $A$ nas bases $\left\{d x^{i}\right\}$ e $\left\{\partial_{i}\right\}$ em alguma coordenada local $\left\{x^{i}\right\}$, ou seja, são as funções

$$
A_{j_{1} \ldots j_{s}}^{i_{1} \ldots i_{r}}=A\left(d x^{i_{1}}, \ldots, d x^{i_{r}}, \partial_{j_{1}}, \ldots, \partial_{j_{s}}\right) .
$$

Com o produto tensorial recuperamos o tensor original $A$ a partir de suas coordenadas locais:

$$
A=A_{j_{1} \ldots j_{s}}^{i_{1} \ldots i_{r}} \partial_{i_{1}} \otimes \ldots \otimes \partial_{i_{r}} \otimes d x^{j_{1}} \otimes \ldots \otimes d x^{j_{s}} .
$$

\footnotetext{
${ }^{26}$ Em termos de coordenadas, temos a expressão usual, por exemplo $C\left(A_{i k}\right)=A_{i}^{i}$, com a soma implícita
} 
Variedades semi-riemannianas Um tensor métrico $g$ numa variedade $\mathscr{M}$ é um campo tensorial do tipo $(0,2)$ simétrico não-degenerado (i.e., $g(V, W)=$ $0, \forall W \in \mathfrak{X}(\mathscr{M}) \Rightarrow V=0$ ) em $\mathscr{M}$, com signatura constante (o que isso significa, mais a frente).

Ou seja, para cada $p \in \mathscr{M} g$ induz um produto escalar $g_{p}$ em $T_{p}(\mathscr{M})$, e o índice de $g_{p}$ é constante para todo $p$. Em coordenadas locais podemos escrever $g_{p}=g_{i j}(p) d x^{i} \otimes d x^{j}$ onde $g_{i j}(p)=g_{p}\left(\partial_{i}, \partial_{j}\right)$, então, para $v=v^{i} \partial_{i}$ e $w=w^{j} \partial_{j}, g(v, w)=g_{i j} v^{i} w^{j}$.

E como $g$ é não-degenerada a matriz formada por $g_{i j}(p)$ é inversível, e sua inversa é $g^{i j}(p)$. Por $g$ ser simétrica temos ainda $g_{i j}=g_{j i}$ (e também para a inversa). A signatura de $g$ é a dupla $(P, N)$, onde $P$ é a quantidade de autovalores positivos de $g_{i j}$ e $N$ é a quantidade de autovalores negativos de $g_{i j}$. Então, se $g$ for uma métrica essas quantidades devem ser constantes em todo ponto da variedade. Nas variedades de interesse para nós (as lorentzianas) a signatura é $(1, n-1)$, onde, claro, $n$ é a dimensão da variedade.

Conexões e Derivadas Uma conexão $\nabla$ na variedade $\mathscr{M}$ é uma função $\nabla: \mathfrak{X}(\mathscr{M}) \times \mathfrak{X}(\mathscr{M}) \rightarrow \mathfrak{X}(\mathscr{M})$ tal que:

(1) $\nabla_{V} W$ é $\mathfrak{F}(\mathscr{M})$-linear em $V$; (2) $\nabla_{V} W$ é $\mathbb{R}$-linear em $W$; (3) $\nabla_{V}(f W)=$ $(V f) W+f \nabla_{V} W, \quad \forall f \in \mathfrak{F}(\mathscr{M})$.

E além disso é possível provar que numa variedade semi-riemanniana $(\mathscr{M}, g)$ há uma única conexão $\nabla$ que satisfaz ainda duas mais propriedades: (3) $[V, W]=\nabla_{V} W-\nabla_{W} V ;(4) X g(V, W)=g\left(\nabla_{X} V, W\right)+g\left(V, \nabla_{X} W\right)$.

No que segue assumiremos que a conexão é a função $\nabla: \mathfrak{X}(\mathscr{M}) \times \mathfrak{X}(\mathscr{M}) \rightarrow$ $\mathfrak{X}(\mathscr{M})$ que satisfaz as quatro propriedades acima, e disso obtemos os resultados familiares de derivadas covariantes, etc. Ou seja, em uma carta local $(\mathscr{U}, h)$ com coordenadas $\left\{x^{i}\right\}$, definimos os símbolos de Christoffel como os valores $\Gamma_{i j}^{k}$ tais que:

$$
\nabla_{\partial_{i}}\left(\partial_{j}\right)=\Gamma_{i j}^{k} \partial_{k}
$$

Nessas coordenadas os símbolos de Christoffel são expressos em termos da métrica por:

$$
\Gamma_{i j}^{k}=\frac{1}{2} g^{k m}\left(\frac{\partial g_{j m}}{\partial x^{i}}+\frac{\partial g_{i m}}{\partial x^{j}}-\frac{\partial g_{i j}}{\partial x^{m}}\right) .
$$

E calculemos, nessas mesmas coordenadas, $\nabla_{U} V$, que chamamos de a derivada covariante de $V$ na direção $U$. Em coordenadas, $U=U^{i} \partial_{i}, V=V^{i} \partial_{i}$.

$$
\begin{gathered}
\nabla_{U^{i} \partial_{i}}\left(V^{j} \partial_{j}\right)=U^{i} \nabla_{\partial_{j}}\left(V^{j} \partial_{j}\right) \\
=U^{i}\left[\left(\partial_{i} V^{j}\right) \partial_{j}+V^{j} \nabla_{\partial_{i}}\left(\partial_{j}\right)\right]=U^{i}\left(\partial_{i} V^{k}+V^{j} \Gamma_{i j}^{k}\right) \partial_{k} .
\end{gathered}
$$


E, em particular:

$$
\nabla_{\partial_{i}}\left(V^{j} \partial_{j}\right)=\left(\frac{\partial V^{k}}{\partial x^{i}}+\Gamma_{i j}^{k} V^{j}\right) \partial_{k}
$$

Por simplicidade usaremos a notação $\nabla_{\partial_{i}}=\nabla_{i}$.

Devemos ainda estender o conceito de derivadas covariantes para tensores em geral, e para isso vamos definir a derivada covariante de uma função e de uma 1-forma, tendo sempre em mente a regra de Leibniz. Talvez, neste ponto, a discussão se torne confusa, pois usaremos o mesmo nome e símbolo para objetos diferentes: definimos a derivada covariante na direção de um certo vetor para funções como uma aplicação $\nabla: \mathfrak{X}(\mathscr{M}) \times \mathfrak{F}(\mathscr{M}) \rightarrow \mathfrak{F}(\mathscr{M})$ e para 1 formas como a aplicação $\nabla: \mathfrak{X}(\mathscr{M}) \times \mathfrak{X}(\mathscr{M})^{*} \rightarrow \mathfrak{X}(\mathscr{M})^{*}$, no entanto, quando definirmos para tensores gerais essa confusão será eliminada, mostrando-se apenas como um caminho para uma generalização do conceito anterior.

A derivada covariante de uma função $f \in \mathfrak{F}(\mathscr{M})$ na direção $v$ é:

$$
\nabla_{V} f=V f
$$

E dada uma 1-forma $\theta$ e um vetor $V, \theta V$ é uma função, então, sua derivada covariante na direção $U$ é $U(\theta V)$. Definimos a derivada covariante $\nabla_{U} \theta$ através da relação:

$$
\left(\nabla_{U} \theta\right)(V)=U(\theta V)-\theta\left(\nabla_{U} V\right)
$$

em uma carta local $\left(\mathscr{U}_{\alpha}, h_{\alpha}\right)$ com coordenadas $\left\{x^{i}\right\}$ temos $\nabla_{V} f=V^{i} \partial_{i} f=$ $V^{i} \partial f / \partial x^{i}$, e usando esse resultado junto com a forma de $\nabla_{U} V$ em coordenadas locais, calculamos:

$$
\begin{gathered}
\nabla_{U} \theta V=U^{i} \partial_{i}\left(\theta_{k} d x^{k}\left(V^{j} \partial_{j}\right)\right)-\theta_{k} d x^{k}\left(U^{i}\left(\partial_{i} V^{l}+V^{j} \Gamma_{i j}^{l}\right) \partial_{l}\right) \\
=U^{i}\left(\partial_{i} \theta_{k}\right) V^{k}+U^{i} \theta_{k} \partial_{i} V^{k}-\theta_{k} U^{i} \partial_{i} V^{k}-\theta_{k} U^{i} V^{j} \Gamma_{i j}^{k} \\
=U^{i}\left(\partial_{i} \theta_{k}-\theta_{k} \Gamma_{i j}^{k}\right) V^{j} .
\end{gathered}
$$

Obtemos, assim, a familiar formula: $\nabla_{i} \theta=\left(\partial_{i} \theta_{k}-\theta_{k} \Gamma_{i j}^{k}\right) d x^{j}$.

Com essas definições e resultados em mãos definimos a derivada covariante, na direção de algum vetor, de um tensor do tipo $(r, s)$ como a aplicação $\nabla: \mathfrak{X}(\mathscr{M}) \times \mathfrak{F}^{r, s}(\mathscr{M}) \rightarrow \mathfrak{F}^{r, s+1}(\mathscr{M})$, definido através da seguinte relação, também inspirada pela relação de Leibniz:

$$
\nabla_{U}(A \otimes B)=\left(\nabla_{U} A\right) \otimes B+A \otimes\left(\nabla_{U} B\right) .
$$

E, claro, como o produto tensorial é uma operação que nos fornece tensores de ordem maior, e com a identificação de $\mathfrak{X}(\mathscr{M})$ e $\mathfrak{X}(\mathscr{M})^{*}$ com tipos de tensores, a relação acima define a derivada covariante para todos os tensores. A 
forma das componentes da derivada covariante de um tensor é uma mistura das componentes das derivadas covariantes de vetores e 1-formas nessas coordenadas, $\nabla_{i} v^{j}=\partial v^{i} / \partial x^{j}+\Gamma_{i k}^{j} v^{k}$ e $\nabla_{i} \theta_{j}=\partial \theta_{j} / \partial x^{i}+\Gamma_{i j}^{k} \theta_{k}$, como é conhecido da relatividade geral.

Por fim mostrarei o que é uma derivada covariante induzida em curvas, para que em paragrafo posterior possamos definir geodésicas e exponenciais em variedades.

Seja $\alpha: I \rightarrow \mathscr{M}$ uma curva, e $V$ um campo vetorial em $\alpha$, isto é, $V \in \mathfrak{X}(\alpha)$ e $V_{\alpha}(t)=(V \circ \alpha)(t) \in T_{\alpha(t)}(\mathscr{M})$. Então, há uma única aplicação $\mathfrak{X}(\alpha) \ni V \mapsto$ $V^{\prime} \in \mathfrak{X}(\alpha)$, que seja $\mathbb{R}$-linear e

$$
(f V)^{\prime}=f^{\prime} V+f V^{\prime}, \quad\left(V_{\alpha}\right)^{\prime}(t)=\nabla_{\alpha^{\prime}(t)} V, \quad \forall f \in \mathfrak{F}(I) .
$$

Seja $\left\{x^{i}\right\}$ coordenadas em torno de $\alpha(t)$ (mais a frente omitiremos o $t$ ), então, $V_{\alpha}(t)=\left.V_{\alpha}(t) x^{i} \partial_{i}\right|_{\alpha(t)}$, e denotemos $V_{\alpha}(.) x^{i}: I \rightarrow \mathbb{R}$ por $V_{\alpha}^{i}$, então, $V_{\alpha}=\left.V_{\alpha}^{i} \partial_{i}\right|_{\alpha}$. Portanto,

$$
V_{\alpha}^{\prime}=\frac{d V_{\alpha}^{i}}{d t} \partial_{i}+V_{\alpha}^{i}\left(\partial_{i}\right)^{\prime}=\frac{d V_{\alpha}^{i}}{d t} \partial_{i}+V_{\alpha}^{i} \nabla_{\alpha^{\prime}}\left(\partial_{i}\right) .
$$

Mas, se $\alpha^{\prime}=\alpha^{\prime k} \partial_{k}=d / d t\left(x^{k} \circ \alpha\right) \partial_{k}$, então, $\nabla_{\alpha^{\prime}}\left(\partial_{i}\right)=d / d t\left(x^{k} \circ \alpha\right) \Gamma_{k i}^{j} \partial_{j}$. Portanto:

$$
V_{\alpha}^{\prime}=\left(\frac{d V_{\alpha}^{j}}{d t}+V_{\alpha}^{i} \frac{d}{d t}\left(x^{k} \circ \alpha\right) \Gamma_{k i}^{j}\right) \partial_{j} .
$$

O campo $V$ é paralelo se $V^{\prime}=0$, ou seja, se as equações $d / d t\left(V_{\alpha}^{j}\right)+$ $\Gamma_{k i}^{j} V_{\alpha}^{i} d / d t\left(x^{k} \circ \alpha\right)=0, j=1, \ldots, n=\operatorname{dim} \mathscr{M}$ forem satisfeitas.

Geodésicas Seja a curva $\alpha: I \rightarrow \mathscr{M}$, tomamos $\alpha^{\prime}$ como sendo o campo vetorial em $\alpha$ (conforme a definição do parágrafo anterior). $\alpha$ é uma geodésica se $\alpha^{\prime}$ for paralelo: $\alpha^{\prime \prime}=0$. Ou seja, em coordenadas locais: $\alpha^{\prime i}=\alpha^{\prime} x^{i}=$ $d / d t\left(x^{i} \circ \alpha\right)$, e denotemos $x^{i} \circ \alpha$ por $\alpha^{i}$, então,

$$
\alpha^{\prime \prime}=\left(\alpha^{\prime}\right)^{\prime}=\left[\frac{d^{2}}{d t^{2}}\left(\alpha^{j}\right)+\Gamma_{k i}^{j} \frac{d \alpha^{i}}{d t} \frac{d \alpha^{k}}{d t}\right] \partial_{j} .
$$

Então, a condição de $\alpha$ ser geodésica é

$$
\frac{d^{2}}{d t^{2}}\left(\alpha^{j}\right)+\Gamma_{k i}^{j} \frac{d \alpha^{i}}{d t} \frac{d \alpha^{k}}{d t}=0 .
$$

Podemos sempre associar a um ponto $p \in \mathscr{M}$ e um $v \in T_{p}(\mathscr{M})$ uma geodésica $\gamma_{v}: I_{v} \rightarrow \mathscr{M}$ tal que $\gamma_{v}\left(t_{0}\right)=p$ e $\gamma_{v}^{\prime}\left(t_{0}\right)=v$, já que isso, em vista da condição de uma curva ser geodésica ser uma equação diferencial ordinária, é equivalente a fornecer as condições iniciais de uma EDO, cujas soluções existem e 
são únicas. É simples ainda mostrar que podemos tomar $I_{v} \supset[0,1]$ como o maior intervalo possível, ou seja, se houver outra geodésica com velocidade inicial $v$ e posição inicial $p$ definida, em, digamos $J \subset I_{v}$, então ela é a restrição de $\gamma_{v}$ à $J$. E dizemos que $t_{0}=0$.

Geodésicas nos maiores intervalos $I_{v}$, como descritos acima, são chamadas de maximais ou inextensíveis. Definimos a aplicação exponencial da seguinte forma: sejam $p \in \mathscr{M}$ e $D_{p}=\left\{v \in T_{p}(\mathscr{M})\right.$ : existem geodésicas $\gamma_{v}$ inextensíveis $\}$, então:

$$
\begin{gathered}
\exp _{p}: D_{p} \rightarrow \mathscr{M}, \\
\exp _{p}(v)=\gamma_{v}(1), \forall v \in D_{p} .
\end{gathered}
$$

Sejam $v \in D_{p}$ e $t, s \in I_{v}$ e $t s \in I_{v}$, a geodésica $s \mapsto \gamma_{v}(t s)$ tem velocidade inicial $t v=t \gamma_{v}^{\prime}(0)$, mas esta é a velocidade inicial que corresponde à geodésica $\gamma_{t v}$, de modo que $\gamma_{t v}(s)=\gamma_{v}(t s)$. Temos, então, o seguinte resultado para exponenciais: $\exp _{p}(t v)=\gamma_{t v}(1)=\gamma_{v}(t)$. Ou seja, a pontos de $T_{p}(\mathscr{M})$, digamos $u$, associamos pontos de $\mathscr{M}$ que são alcançados por geodésicas $u$. É possível mostrar ainda que $\exp _{p}$ pode atuar como um difeomorfismo entre vizinhanças em $T_{p}(\mathscr{M})$ e vizinhanças de $\mathscr{M}$ (vide, e.g., Capítulo 3 de [25]): para cada ponto $p \in \mathscr{M}$ há uma vizinhança $\mathscr{U}^{\prime} \subset T_{p}(\mathscr{M})$ de 0 na qual $\exp _{p}$ é um difeomorfismo em uma vizinhança $\mathscr{U}=\left.\exp _{p}\right|_{\mathscr{U}^{\prime}}\left(D_{p}\right)$ de $p$.

Dizemos que um subconjunto $E \subset T_{p}(\mathscr{M})$ é starshaped em torno de 0 se $v \in E \Rightarrow t v \in E, \forall t \in[0,1]$. Se $\mathscr{U}^{\prime}$ é starshaped em torno de zero (sendo, então, uma vizinhança de zero), chamamos $\mathscr{U}=\left.\exp _{p}\right|_{\mathscr{U}^{\prime}}\left(D_{p}\right)$ de vizinhança normal em torno de $p=\exp _{p}\left(\left.t\right|_{t=0} v\right)=\gamma_{v}(0)=p$. Um subconjunto $\mathscr{V}$ de $\mathscr{M}$ é (geodésicamente) convexo se todos os seus pontos possuirem uma vizinhança norma 27. Vizinhanças normais nos serão úteis quando for necessário relacionar objetos de $T_{p}(\mathscr{M})$ com objetos de $\mathscr{M}$ (por exemplo, quando formos construir soluções locais da equação de onda a partir de soluções num espaço de Minkowski).

Um resultado importante sobre exponenciais é o lema de Gauss, que nos diz que exponenciais são isometrias ${ }^{28}$ em direções radiais entre $T_{p}(\mathscr{M})$ e $\mathscr{M}$ : Seja $v \in D_{p}$ e $u_{1}, u_{2} \in T_{v}\left(T_{p}(\mathscr{M})\right) \simeq T_{p}(\mathscr{M})$, tal que $\exists t \in \mathbb{R}, u_{1}=t v$, então,

$$
g_{\exp _{p}(v)}\left(d\left(\exp _{p}\right)_{v}\left(u_{1}\right), d\left(\exp _{p}\right)_{v}\left(u_{2}\right)\right)=g_{p}\left(u_{1}, u_{2}\right) .
$$

Operadores diferenciais O gradiente de uma função $f \in \mathfrak{F}(\mathscr{M})$ é o vetor $\operatorname{grad} f \in \mathfrak{X}(\mathscr{M})$ tal que $(d f)(U)=g(\operatorname{grad} f, U)$. Em coordenadas lo-

\footnotetext{
${ }^{27} \mathrm{Em}$ variedades Lorentzianas todos os seus pontos possuem uma vizinhança normal, e cada cobrimento possui um refinamento cujos abertos são convexos

${ }^{28} \mathrm{Uma}$ isometria entre duas variedades $(\mathscr{M}, g)$ e $\left(\mathscr{N}, g^{\prime}\right)$ é um difeomorfismo $\phi: \mathscr{M} \rightarrow$ $\mathscr{N}$ tal que $g_{\phi(p)}^{\prime}\left(d \phi_{p}(u), d \phi_{p}(v)\right)=g_{p}(u, v)$
} 
cais: $g(\operatorname{grad} f, U)=g_{i j}(\operatorname{grad} f)^{i} U^{j}$ e $d f=\partial_{i} f d x^{i}$ e $d x^{i}(X)=X^{i}$, então, $g_{i j}(\operatorname{grad} f)^{j} X^{i}=\partial f / \partial x^{i} X^{i}$, portanto, $(\operatorname{grad} f)^{j}=g^{i j} \partial f / \partial x^{i}$.

O divergente de um vetor $V$ é a contração de sua derivada covariante: $\mathfrak{F}(\mathscr{M}) \ni$ $\operatorname{div} V=C(\nabla V)$. Em coordenadas locais:

$$
\operatorname{div} V=C\left(\sum_{i} \nabla_{i} V_{k}\right)=\sum_{i}\left(\frac{\partial V^{i}}{\partial x^{i}}+\Gamma_{i j}^{i} V^{j}\right) .
$$

O laplaciano de uma função $f \in \mathfrak{F}(\mathscr{M})$ é o divergente de seu gradiente: $\square f=\operatorname{div}(\operatorname{grad} f)$. Em coordenadas locais:

$$
\square f=\frac{\partial}{\partial x^{i}}\left(g^{i j} \frac{\partial f}{\partial x^{j}}\right)+g^{j k} \Gamma_{i j}^{i} \frac{\partial f}{\partial x^{k}} .
$$

Utilizando ainda o seguinte resultado para os símbolos de Christoffel [36]: $\Gamma_{i k}^{i}=1 / \sqrt{g} \partial_{k} \sqrt{g}$, onde $g=\operatorname{det}\left(g_{i j}\right)$, escrevemos o laplaciano como:

$$
\square=\frac{1}{\sqrt{g}} \partial_{i}\left(g^{i j} \sqrt{g} \partial_{j}\right)
$$

\subsubsection{Distribuições em variedades}

No que segue será importante definir distribuições em variedades. Já sabemos como distribuições funcionam em $\mathbb{R}^{n}$, mas façamos uma breve recordação. Seja $X$ um aberto de $\mathbb{R}^{n}, f: X \rightarrow \mathbb{R}$ é $C^{k}$ se, e somente se $\partial_{i_{1}} \cdots \partial_{i_{j}} f$, $j \leq k$ existirem e forem continuas. Usaremos a notação de multi-índice: $\partial^{\alpha} f=\partial_{1}^{\alpha_{1}} \cdots \partial_{n}^{\alpha_{n}} f, \alpha=\left(\alpha_{1}, \ldots, \alpha_{n}\right)$, e $|\alpha|=\sum_{j=1}^{n} \alpha_{j}$ é a ordem de $\partial^{\alpha}$. Por exemplo, para $\alpha=(2,1,3)$ temos: $\partial^{\alpha} f=\partial_{x}^{2} \partial_{y} \partial_{z}^{3} f$.

Chamamos $C^{k}(X)$ o espaço das funções $C^{k}$ em $X$. Para $k=0, C^{0}(X)=$ $C(X)$ é o espaço das funções continuas em $X$. O espaço $C^{\infty}(X)$ é definido da forma usual. Definimos o suporte de $f$ como $\operatorname{supp} f=\overline{\{x \in X: f(x) \neq 0\}}$, ou seja, $\operatorname{supp} f$ é o menor fechado no qual $f$ é não nula. $C_{0}^{k}(X)$ é o espaço das funções $C^{k}$ em $X$ com suporte compacto. ${ }^{29}$.

Uma distribuição $u$ em $X$ é um funcional linear em $C_{0}^{\infty}(X)$ tal que para todo compacto $K \subset X$ existem constantes $C, k$ tais que

$$
|u(f)| \leq C \sum_{|\alpha| \leq k} \sup \left|\partial^{\alpha} f\right|, \quad \forall f \in C_{0}^{\infty}(X) .
$$

O conjunto das distribuições em $X$ é denotado $\mathscr{D}^{\prime}(X)$, e o conjunto das distribuições em $X$ com suporte ${ }^{30}$ compacto é denotado por $\mathscr{E}^{\prime}(X) \subset \mathscr{D}^{\prime}(X)$.

\footnotetext{
${ }^{29}$ Em variedades usaremos, baseado na notação anterior, $\mathfrak{F}_{0}(\mathscr{M})$ como o espaço das funções em $\mathfrak{F}(\mathscr{M})$ com suporte compacto

${ }^{30} \mathrm{O}$ suporte de uma distribuição $u$ é o complemento do maior aberto no qual $u$ se anula, ou seja, $X \supset \operatorname{supp} u=A^{c}$, onde $A$ é o maior aberto no qual $u(f)=0, \forall f \operatorname{com} \operatorname{supp} f \subset A$
} 
A derivada de uma distribuição $u \in \mathscr{D}^{\prime}(X)$ é definida pela seguinte relação $\left(\partial_{i} u\right)(f)=-u\left(\partial_{i} f\right), \forall f \in C_{0}^{\infty}(X)$, e é também uma distribuição. Portanto, $\left(\partial^{\alpha} u\right)(f)=(-1)^{|\alpha|} u\left(\partial^{\alpha} f\right)$. E a multiplicação por uma função é definida por: $\forall f, g \in C_{0}^{\infty},(g u)(f)=u(g f)$.

O conjunto $C^{\infty}(X)$ (ou até $C(X)$ ) está, em certo sentido, contido em $\mathscr{D}^{\prime}(X)$ se associarmos à toda $f \in C^{\infty}(X)$ a distribuição $f \in \mathscr{D}^{\prime}(X)$ caracterizada por: $f(\phi)=\int f(x) \phi(x) d x, \forall \phi \in C_{0}^{\infty}(X)$.

Dessa identificação podemos esclarecer a definição de derivadas de distribuições: seja um operador diferenciável $P$, chamamos de seu adjunto formal o operador $P^{*}$ tal que $\int f(x)(P \phi)(x) d x=\int\left(P^{*} f\right)(x) \phi(x) d x$. Caso $P=\partial_{i}$

$$
\int f(x)\left(\partial_{i} \phi\right)(x) d x=-\int\left(\partial_{i} f\right)(x) \phi(x) d x+\int \partial_{i}(f \phi)(x) d x .
$$

E como $\phi$ tem suporte compacto, a última integral vai a zero, de modo que $P^{*}=-\partial_{i}$, o que nos leva a definir a distribuição $\partial_{i} f$ como $\left(\partial_{i} f\right)(\phi)=$ $-f\left(\partial_{i} \phi\right)$.

Distribuições em variedades são objetos que agem em $\mathfrak{F}_{0}(\mathscr{M})$ que localmente podem ser identificados como distribuições em abertos $X \subset \mathbb{R}^{n}$. A definição de distribuição a seguir (essencialmente como é definida em [22]) é inspirada no fato de $f \in \mathfrak{F}(\mathscr{M})$ poder ser completamente caracterizada por "componentes" locais, da seguinte forma: se $f \in \mathfrak{F}(\mathscr{M})$, e dado o atlas $\left\{\left(\mathscr{U}_{\lambda}, h_{\lambda}\right)\right\}$, temos funções em $\mathbb{R}^{n}$ que são as componentes de $f$ num sistema de coordenadas: $f_{\lambda}=f \circ h_{\lambda}^{-1}: h_{\lambda}\left(\mathscr{U}_{\lambda}\right) \subset \mathbb{R}^{n} \rightarrow \mathbb{R}$, que é uma função em $\mathfrak{F}\left(h_{\lambda}\left(\mathscr{U}_{\lambda}\right)\right)$. E para duas cartas $\left(\mathscr{U}_{\alpha}, h_{\alpha}\right)$ e $\left(\mathscr{U}_{\beta}, h_{\beta}\right)$, em $\mathscr{U}_{\alpha} \cap \mathscr{U}_{\beta}$ temos

$$
f=f_{\alpha} \circ h_{\alpha}=f_{\beta} \circ h_{\beta} .
$$

Então, em $h_{\beta}\left(\mathscr{U}_{\alpha} \cup \mathscr{U}_{\beta}\right), f_{\beta}=f_{\alpha} \circ\left(h_{\alpha} \circ h_{\beta}^{-1}\right)$.

É, então, possível mostrar que dado o sistema $\left\{f_{\alpha}\right\}, f_{\alpha}$ suaves, tal que para quaisquer $\mathscr{U}_{\alpha}$ e $\mathscr{U}_{\beta}$ com intersecção não vazia, valha a relação acima, há uma única $f \in \mathfrak{F}(\mathscr{M})$ correspondente - basta juntar a coleção $\left\{f_{\alpha}\right\}$ através de uma partição da unidade subordinada ao cobrimento $\left\{\left(\mathscr{U}_{\alpha}, h_{\alpha}\right)\right\}$.

Definimos distribuições em $\mathscr{M}$ da seguinte forma: seja $\left\{\left(\mathscr{U}_{\alpha}, h_{\alpha}\right)\right\}$ um atlas em $\mathscr{M}$. Uma distribuição em $\mathscr{M}$ é o objeto $u$ associado à coleção $\left\{u_{\alpha} \in\right.$ $\left.\mathscr{D}^{\prime}\left(h_{\alpha}\left(\mathscr{U}_{\alpha}\right)\right)\right\}$ de distribuições locais, tais que para quaisquer cartas locais $\left(\mathscr{U}_{\alpha}, h_{\alpha}\right)$ e $\left(\mathscr{U}_{\beta}, h_{\beta}\right)$, vale a relação, $\forall g \in C_{0}^{\infty}\left(\mathbb{R}^{n}\right)$

$$
u_{\beta}(g)=u_{\alpha}\left(g \circ h_{\alpha} \circ h_{\beta}^{-1}\right) .
$$

Podemos falar da "componente" local de $u$, para qualquer carta $\left(\mathscr{U}_{\alpha}, h_{\alpha}\right)$, de uma distribuição em $\mathscr{M}$ como sendo o funcional $u^{\mathscr{U}_{\alpha}}: \mathfrak{F}_{0}(\mathscr{M}) \rightarrow \mathbb{R}$ que é 
dada por

$$
u^{\mathscr{U}_{\alpha}}(f)=u_{\alpha}\left(f \circ h_{\alpha}^{-1}\right), \quad \forall f \in \mathfrak{F}_{0}(\mathscr{M}), \quad u_{\alpha} \in \mathscr{D}^{\prime}\left(h_{\alpha}\left(\mathscr{U}_{\alpha}\right)\right) .
$$

Claro, essa definição local pode ser estendida a uma definição global, juntando cada pedaço através da partição da unidade. Isso ficará claro no exemplo a seguir.

Exemplo A distribuição $\delta_{p}: \mathfrak{F}_{0}(\mathscr{M}) \rightarrow \mathbb{R}$ é caracterizada pela relação $\delta_{p}(f)=f(p), \forall f \in \mathfrak{F}_{0}(\mathscr{M})$. De fato, seja $p_{\alpha}=h_{\alpha}(p)$, para toda carta $\left(\mathscr{U}_{\alpha}, h_{\alpha}\right)$ que contém $p$. O sistema local $\left\{\delta_{p_{\alpha}}\right\}$ que define $\delta_{p}$ são as deltas usuais: $\delta_{p_{\alpha}}\left(f \circ h_{\alpha}^{-1}\right)=\left(f \circ h_{\alpha}^{-1}\right)\left(p_{\alpha}\right)=f\left(h_{\alpha}^{-1}\left(p_{\alpha}\right)\right)=f(p)$. E se a carta (digamos, $\left.\left(\mathscr{U}_{\beta}, h_{\beta}\right)\right)$ não contém $p$, tomamos $u_{\beta}=0$. Unimos o sistema $\left\{u_{\lambda}\right\}$ através da partição da unidade $\left\{g_{\lambda}\right\}$ subordinada ao atlas usado (e, portanto, $\operatorname{supp} g_{\lambda} \subset \mathscr{U}_{\lambda}$, portanto, da definição de partição da unidade: $\sum_{\alpha} g_{\alpha}(p)=1$, com $\alpha$ percorrendo as cartas que contém $p$ ). Temos, então, o objeto global que podemos chamar de uma distribuição em $\mathscr{M}: u(f)=\sum_{\lambda} u_{\lambda}\left(\left(g_{\lambda} f\right) \circ h_{\lambda}^{-1}\right)$.

Conservando a notação utilizada até agora, para denotar objetos como funções, vetores, etc., em $\mathscr{M}$, denotaremos o conjunto de distribuições em $\mathscr{M}$ por $\mathfrak{D}^{\prime}(\mathscr{M})$, e o conjunto de distribuições com suporte compacto em $\mathscr{M}$ por $\mathfrak{E}^{\prime}(\mathscr{M})$. E da mesma forma que, para $X \subset \mathbb{R}^{n}$, definimos o que é o suporte de uma distribuição, definimos o suporte de uma distribuição em $\mathscr{M}$ como o complemento do maior aberto no qual ela se anula. E também, analogamente ao caso euclidiano, identificamos $\mathfrak{F}(\mathscr{M})$ como subconjunto de $\mathfrak{D}^{\prime}(\mathscr{M})$, associando a cada $u \in \mathfrak{F}(\mathscr{M})$ a distribuição $u \in \mathfrak{D}^{\prime}(\mathscr{M})$ segundo a relação

$$
\mathfrak{F}_{0}(\mathscr{M}) \ni f \mapsto \int_{\mathscr{M}} u(x) f(x) d V,
$$

onde $\mathfrak{F}_{0}(\mathscr{M})$ é o conjunto das funções suaves de $\mathscr{M}$ com suporte compacto, e $d V$ é a medida associada à métrica $g$, que em coordenadas locais é $d V=$ $\sqrt{g} d^{4} x$, onde $\sqrt{g}=\sqrt{|\operatorname{det} g|}$, e escrevemos isso como $u(f)=\int u(x) f(x) d V$. Como foi feito no caso de distribuições em $\mathbb{R}^{n}$ podemos definir operadores diferenciais agindo em distribuições definidas em variedades olhando para o adjunto formal de um operador diferencial. Ou seja, dado um operador diferencial $P$, definimos seu adjunto formal como sendo o operador $P^{*}$, tal que $\int u(x)\left(P^{*} f\right)(x) d V=\int(P u)(x) f(x) d V$. Dessa definição definimos um operador diferencial $P$ que age na distribuição $u$ como sendo a distribuição $P u$, caracterizada por $(P u)(f)=u\left(P^{*} f\right)$.

O laplaciano é $\square: \mathfrak{F}(\mathscr{M}) \rightarrow \mathfrak{F}(\mathscr{M})$, e como ele é o operador diferencial que mais nos vai ocupar, não precisaremos definir distribuições tensoriais 
como faz Friedlander em [16]. Vejamos, então, como age a distribuição $\square u$ : sabemos que, pelas mesmas contas feitas no caso de distribuições reais, $\partial_{i}^{*}=$ $-\partial_{i}$, então, sendo $d V=\sqrt{g} d^{n} x$ :

$$
\begin{gathered}
\int_{\mathscr{U}} \square u f d V=\int_{\mathscr{U}} \frac{1}{\sqrt{g}}\left(\partial_{i}\left(g^{i j} \sqrt{g} \partial_{j} u\right)\right) f \sqrt{g} d^{n} x=-\int_{\mathscr{U}}\left(g^{i j} \sqrt{g} \partial_{j} u\right) \partial_{i} f d^{n} x= \\
=\int_{\mathscr{U}} \frac{1}{\sqrt{g}}\left(\partial_{j}\left(g^{i j} \sqrt{g} \partial_{i} f\right)\right) u \sqrt{g} d^{n} x
\end{gathered}
$$

Então, $\square^{*}=\square$, e $(\square u)(f)=u(\square f)$.

\subsubsection{Estrutural Causal}

Estrutura causal no espaço de Minkowski: No espaço de Minkowski classificamos os eventos $u \in \mathbb{R}^{4}$ em tipo-tempo, tipo-luz, causal e tipo espaço se, respectivamente, $u^{2}=u_{\mu} u^{\mu}>0, u_{\mu} u^{\mu}=0, u_{\mu} u^{\mu} \geq 0$ e $u_{\mu} u^{\mu}<0$. Dois eventos $u, v \in \mathbb{R}^{4}$ tem separação tipo-tempo, tipo-luz, etc., se $(u-v)^{2}>0$, $(u-v)^{2}=0$, etc.

Sabemos o que isso significa no contexto da relatividade especial: os eventos com separação causal estão causalmente ligados, ou seja, eles podem ser influenciados (e.g., um pode observar o outro), enquanto que na separação espacial não pode haver influência entre os eventos. É essa a fonte da primeira parte dos axiomas de Haag-Kastler: quando é dito que observáveis de regiões espacialmente separadas comutam, quer-se expressar nisso a independência desses observáveis.

Vetores causais formam dois cones em torno do eixo temporal, que são separados conforme o sinal de $u^{0}$ : se $u^{0}>0$ dizemos que $u$ é futuro-direcionado, e se $u^{0}<0$ dizemos que $u$ é passado-direcionado. A interpretação dessa definição (que no caso dos espaços de Minkowski é clara) se torna mais evidente se considerarmos separações entre eventos: sejam $u, v$ dois eventos cuja separação é causal, dizemos que $u-v$ é futuro-direcionado se $\left(u^{0}-v^{0}\right)>0$, e isso é equivalente a dizer que o evento $u$ é posterior ao evento $v$. Definimos, então, as seguintes relações entre eventos:

$y \ll x \Leftrightarrow(x-y)^{2}>0$ e $\left(x^{0}-y^{0}\right)>0$

$y<x \Leftrightarrow(x-y)^{2} \geq 0$ e $\left(x^{0}-y^{0}\right)>0 ;$

$y \leq x \Leftrightarrow y<x$ ou $y=x$.

Nesse ponto passaremos à variedades mais gerais, pois é aparente que as motivações para as seguintes definições já estão claras.

Estrutura causal em variedades Lorentzinas: Seja $(\mathscr{M}, g)$ uma variedade diferenciável Lorentziana. Dizemos que dois vetores $x, y \in T_{p}(\mathscr{M})$ tem separação 
tipo-tempo, tipo-luz, causal ou espacial se, respectivamente, $g(x, y)>0$, $g(x, y)=0, g(x, y) \geq 0$ e $g(x, y)<0$. Podemos classificar os vetores $x \in T_{p}(\mathscr{M})$ causais em duas classes, $C_{p}^{+}$e $C_{p}^{-}$, caracterizadas pelo sinal de $x^{0}$ :

$C_{p}^{+}=\left\{x \in T_{p}(\mathscr{M}): g_{p}(x, x) \geq 0\right.$ e $\left.x^{0}>0\right\}$, $C_{p}^{-}=\left\{x \in T_{p}(\mathscr{M}): g_{p}(x, x) \geq 0\right.$ e $\left.x^{0}<0\right\}$.

Essa distinção é análoga à distinção feita entre o cone futuro e o cone passado no espaço de Minkowski. Um vetor causal $x$ é dito ser futuro-direcionado se $x \in C_{p}^{+}$e é passado-direcionado se $x \in C_{p}^{-}$. É claro, podemos falar em vetores tipo-tempo ou tipo-luz que são futuro- ou passado-direcionados, sendo esses os constituintes dos subconjuntos óbvios de $C_{p}^{+}$e $C_{p}^{-}$, respectivamente. Essas definições por differentiae, que sempre podem ser feitas localmente, define uma orientação temporal local. Mas é razoável também que a variedade possua uma ordem temporal global, dizemos, então, que uma variedade diferenciável lorentziana é orientável temporalmente se for possível classificar os vetores causais de $T(\mathscr{M})$ entre futuro-direcionados ou passado-direcionados de uma forma continua. Ou, de forma mais precisa: $(\mathscr{M}, g)$ é orientável temporalmente se, e somente se, existir um campo vetorial suave tipo-tempo $\tau \in \mathfrak{X}(\mathscr{M})$.

Dizemos que uma $(\mathscr{M}, g)$ que é orientável temporalmente é um espaço-tempo. Passemos agora à algumas definições a mais, que por fim nos permitirão definir de forma precisa um espaço-tempo suficientemente razoável (de tal forma que poderemos até achar soluções globais para equações diferenciais lineares).

Uma curva $\gamma: I \rightarrow \mathscr{M}$, onde $I \subset \mathbb{R}$ é um intervalo nos reais, é dita ser uma curva (tipo-tempo, tipo-luz, causal) futuro-direcionada de $p$ à $q$ se, para $a, b \in I, a<b, \gamma(a)=p, \gamma(b)=q$ e $\gamma^{\prime}(t)$ forem, para todo $t$, vetores (tipo-tempo, tipo-luz, causais) futuro-direcionados. Analogamente definimos curvas passado-direcionadas. E também:

$p \ll q \Leftrightarrow$ há uma curva tipo-tempo futuro-direcionada de $p$ à $q$,

$p<q \Leftrightarrow$ há uma curva causal futuro-direcionada de $p$ à $q$,

$p \leq q \Leftrightarrow p<q$ ou $p=q$.

Definimos agora os seguintes conjuntos, que são apenas as coleções de pontos que tem certa relação causal com um ponto dado:

$I^{+}(p)=\{q \in \mathscr{M}: p \ll q\}$ futuro cronológico de $p$,

$I^{-}(p)=\{q \in \mathscr{M}: q \ll p\}$ passado cronológico de $p$,

$J^{+}(p)=\{q \in \mathscr{M}: p \leq q\}$ futuro causal de $p$,

$J^{-}(p)=\{q \in \mathscr{M}: q \leq p\}$ passado causal de $p$.

Fazendo uniões desses conjuntos obtemos futuros e passados de regiões do espaço-tempo, por exemplo, seja $S \subset \mathscr{M}, I^{+}(S)=\cup_{p \in S} I^{+}(p)$. E ainda po- 
demos definir, por exemplo, $I_{S}^{+}(p)=\{q \in S: p \ll q\}^{31}$

O principal uso das exponenciais em espaços-tempos é devido a sua capacidade de relacionar o futuro ou passado de um ponto em espaços-tempos de Minkowski (que são identificados com os espaços tangentes) com o futuro ou passado em espaços-tempos Lorentzianos (nos quais os espaços tangentes supracitados são tangentes). Denotemos $\mathcal{I}^{ \pm}$e $\mathcal{J}^{ \pm}$os futuro(passado)cronológico e futuro(passado)-causal num espaço-tempo de Minkowski $\left(\mathbb{R}^{n}, \eta\right)$, e $I^{ \pm}$e $J^{ \pm}$os futuro(passado)-cronológico e futuro(passado)-causal num espaçotempo Lorentziano $(\mathscr{M}, g)$. A relação entre esses conjuntos é estabelecida pelo seguinte teorema (de [2]): Seja o espaço-tempo Lorentziano $(\mathscr{M}, g)$ e $p \in \mathscr{M}$. Seja $S^{\prime} \subset T_{p}(\mathscr{M})$ um conjunto aberto starshaped em torno de 0, e $S \subset \mathscr{M}$ uma vizinhança normal em torno de $p$, essas regiões são relacionadas pelo difeomorfismo $\left.\exp _{p}\right|_{S^{\prime}}: S^{\prime} \rightarrow S$. Então, valem as seguintes relações:

$$
\begin{aligned}
I_{S}^{ \pm}(p) & =\exp _{p}\left(\mathcal{I}^{ \pm}(0) \cap S^{\prime}\right), \\
J_{S}^{ \pm}(p) & =\exp _{p}\left(\mathcal{J}^{ \pm}(0) \cap S^{\prime}\right) .
\end{aligned}
$$

Por fim podemos passar ao conjunto final de definições, que nos informará o que é um espaço globalmente hiperbólico e o que é uma superfície de Cauchy, dois conceitos centrais no estudo das equações diferenciais que nos fornecerão os operadores que formam as álgebras CCR e CAR.

Localmente um espaço-tempo se comporta como um familiar espaço-tempo de Minkowski (em particular, sua estrutura causal lembra um espaço-tempo de Minkowski, vide, e.g., Teorema 8.1.2 de [34]). Mas ainda gostaríamos que a estrutura causal global satisfizesse algumas condições, chamadas condições de causalidade. Por exemplo, não é razoável que uma curva causal possa voltar a si mesma, caso isso aconteça a solução de uma equação que depende de condições iniciais não será consistente (de fato, nesse caso o próprio problema de resolver equações que dependem de condições iniciais não parece bem definido). Vejamos mais algumas definições:

Seja uma curva causal $\gamma: I \rightarrow \mathscr{M}$ e sejam $a=\inf I$ e $b=\sup I$, dizemos que $p \in \mathscr{M}$ é um ponto final passado de $\gamma$ se toda sequencia $\left(t_{k}\right) \subset I$ tal que $t_{k} \rightarrow a$ implica que $\gamma\left(t_{k}\right) \rightarrow p$. Analogamente, dizemos que $p \in \mathscr{M}$ é um ponto final futuro de $\gamma$ se para toda $\left(t_{k}\right) \subset I$ que converge a $b$ implicar que $\gamma\left(t_{k}\right) \rightarrow p$. Uma curva que não contém pontos finais é uma curva inextensível. Uma curva causal $\gamma: I \rightarrow \mathscr{M}$ é dita ser fechada se houverem $t_{1}, t_{2} \in I$, $t_{1} \neq t_{2}$, tais que $\gamma\left(t_{1}\right)=\gamma\left(t_{2}\right)$. E $\gamma$ é dita ser quase fechada se para algum ponto $p \in \mathscr{M}$ e para toda vizinhança $U$ de $p$ houverem $t_{1}, t_{2} \in I, t_{1} \neq t_{2}$,

\footnotetext{
${ }^{31}$ Se $S$ for conexo e aberto ele é em si um espaço-tempo cuja métrica é a métrica de $\mathscr{M}$ restrita à $S$.
} 
tais que $\gamma\left(t_{1}\right), \gamma\left(t_{2}\right) \in U$.

Um espaço-tempo $(\mathscr{M}, g)$ satisfaz a condição de causalidade se não contém nenhuma curva causal fechada, e satisfaz a condição forte de causalidade se não contém nenhuma curva causal quase fechada.

Um espaço-tempo $(\mathscr{M}, g)$ é globalmente hiperbólico se satisfaz a condição forte de causalidade e se $J^{+}(p) \cap J^{-}(q)$ for compacto, $\forall p, q \in \mathscr{M}, p<q$.

A condição de $J^{+}(p) \cap J^{-}(q)$ ser compacto requer um esclarecimento de sua motivação. O conjunto $J^{+}(p) \cap J^{-}(q)$, para $p<q$ (caso contrario ele seria vazio), é o menor conjunto que contém todas as curvas causais futurodirecionadas de $p$ à $q$, e é possível mostrar (prova no capitulo 14 de [25]) que se $\mathscr{M}$ satisfizer a condição forte de causalidade e $J^{+}(p) \cap J^{-}(q)$ for compacto, então, há a maior geodésica causal em $J^{+}(p) \cap J^{-}(q)$ que une $p$ à $q$. De modo que num espaço globalmente hiperbólico todos os pontos que estão em uma relação causal podem ser unidos por uma geodésica causal. Dizemos que um subconjunto $A \subset \mathscr{M}$ é causal se seu fecho está contido num subconjunto convex ${ }^{32} \mathcal{A}$ e se, em certo sentido, seu fecho imita essa propriedade de espaços globalmente hiperbólicos, ou, de forma mais precisa: se para todo $p, q \in \bar{A}, J_{\mathcal{A}}^{+}(p) \cap J_{\mathcal{A}}^{-}(q)$ for contido em $\bar{A}$ e compacto.

Por fim, um conjunto $\mathcal{S}$ de $\mathscr{M}$ é uma superfície de Cauchy se toda curva inextensível tipo-tempo de $\mathcal{S}$ passar por $\mathcal{S}$ apenas uma vez. Isso implica que quaisquer dois pontos de $\mathcal{S}$ nunca podem ser unidos através de uma curva tipo-tempo. Outras propriedades importantes de $\mathcal{S}$ (novamente, para provas fazemos referências à [25]) é que $\mathcal{S}$ é uma hipersuperfície; toda curva causal de $\mathscr{M}$ passa por $\mathcal{S}$ e as superfícies de Cauchy de $\mathscr{M}$ são homeomorfas entre si.

Um fato crucial sobre espaços-tempos globamente hiperbólicos $(\mathscr{M}, g)$ é que $\mathscr{M}$ possui necessariamente uma superfície suave de Cauchy. E ainda $(\mathscr{M}, g)$ é isométrico à $\left(\mathbb{R} \times \mathcal{S}, \beta d t^{2}-g_{t}\right)$, onde $\mathcal{S}$ é uma superfície de Cauchy em $\mathscr{M}$, $\beta: \mathbb{R} \times \mathcal{S} \rightarrow(0, \infty)$ é uma função suave, $t: \mathbb{R} \times \mathcal{S} \rightarrow \mathbb{R}$ é a projeção natural e $g_{t}$ é uma métrica Riemanniana em $\mathcal{S}$ que depende suavemente de $t$.

Se $\phi: \mathscr{M} \rightarrow \mathbb{R} \times \mathcal{S}$ for a isometria entre essas variedades, a composição $\mathfrak{F}(\mathscr{M}) \ni \tau=t \circ \phi: \mathscr{M} \rightarrow \mathbb{R}$ é uma função tempo de Cauchy, ou seja, seu gradiente $\operatorname{grad}(\tau) \in \mathfrak{X}(\mathscr{M})$ é tipo-tempo e futuro-direcionado em todo ponto, e ainda toda superficie de nivel de $\tau$ é uma superfície tipo-espaço de Cauchy, e com essas funções de tempo de Cauchy é possível mostrar que o espaço-tempo globalmente hiperbólico $(\mathscr{M}, g)$ é formado por uma foliação de superfícies de Cauchy. Estes resultados são relativamente recentes, e são demonstrados em [6], [7] e [8].

\footnotetext{
${ }^{32}$ Lembrando que dizemos que um subconjunto é convexo se todos os seus pontos possuirem uma vizinhança normal
} 


\subsection{A Equação de Onda num Espaço-tempo Global- mente Hiperbólico}

Como vimos nas seções anteriores as álgebras CCR surgem naturalmente de problemas da mecânica quântica, e vimos que uma álgebra CCR abstrata satisfaz condições que a tornam candidatas para álgebras que descrevem operadores locais, segundo os axiomas de Haag-Kastler. O proposito geral desta seção é montar a estrutura e os objetos necessários para a construção de uma álgebra CCR abstrata com um conteúdo físico, especificando a forma simplética - que é usada na definição de uma álgebra de Weyl e que contém em si um conteúdo relativístico crucial, sem o qual não poderíamos falar em causalidade nos axiomas de Haag-Kastler - através da solução da equação de onda num espaço-tempo globalmente hiperbólico.

Para isso vou indicar como podem haver soluções globais para a equação de onda num espaço-tempo globalmente hiperbólico, e como essas soluções são expressas em termos de operadores de Green (avançados e retardados). Primeiro, falaremos sobre soluções em espaços-tempos de Minkowski, em seguida soluções locais em espaços-tempos curvos, em seguida discutiremos a existência e unicidade da solução global em espaços-tempos globalmente hiperbólicos, e por fim os operadores de Green.

O texto que segue pode parecer beirar um cento, e de fato, não é possível incluir todos os detalhes e trazê-los à uma unidade em um texto tão breve. Então, para as provas que forem omitidas aqui, apontamos em direção aos textos de Bär, Ginoux e Pfäffle, [2], [3], que tratam de equações mais geraise que são, aliás, os textos utilizados para a formação desta seção.

\subsubsection{Formulações do Problema}

No estudo de soluções da equação de onda é comum, ao invés de contemplar a equação como uma equação diferencial, estudar uma equação no sentido distribucional, relacionada ao problema original pela solução fundamental. Seja $P$ um operador d'Alembertiand ${ }^{33}$ em $(\mathscr{M}, g)$, a equação de interesse é

$$
P u=f,
$$

onde $u, f \in \mathfrak{F}(\mathscr{M})$. Uma solução fundamental de $P$ em $x \in \mathscr{M}$ é a distribuição $F_{x}$ tal que

$$
P F_{x}=\delta_{x} .
$$

\footnotetext{
${ }^{33}$ Aqui nos restringiremos ao operador $P=\square+m^{2}$, para uma definição mais geral vide [2].
} 
Dada uma solução fundamental de $P$ em $x$, em uma região $\mathscr{U}$, a solução de (7), no sentido distribucional, é dada por $u(\phi)=\int_{\mathscr{U}} f(x) F_{x}(\phi) d V, \forall \phi \in$ $\mathfrak{F}_{0}(\mathscr{M})$, já que:

$$
\begin{gathered}
(P u)(\phi)=u\left(P^{*} \phi\right)=\int_{\mathscr{U}} f(x) F_{x}\left(P^{*} \phi\right) d V=\int_{\mathscr{U}} f(x)\left(P F_{x}\right)(\phi) d V \\
=\int_{\mathscr{U}} f(x) \phi(x) d V .
\end{gathered}
$$

Ou seja, $P u=f$, onde $f$ é a distribuição $\phi \mapsto \int_{\mathscr{U}} f(x) \phi(x) d V$. Há ainda uma restrição um tanto natural a se fazer para soluções fundamentais, a saber, impomos soluções com suporte contido no futuro ou passado causal do ponto onde ela é calculada:

$$
\operatorname{supp}\left(F_{x}^{ \pm}\right) \subset J^{ \pm}(x) .
$$

Um problema de Cauchy é um problema que exige a solução de uma equação de segunda ordem, com as condições iniciais dadas em um subconjunto do espaço-tempo, a saber, em uma superfície de Cauchy. Mais precisamente: sejam $(\mathscr{M}, g)$ um espaço-tempo globalmente hiperbólico, $P$ um operador d'Alembertiano e $\mathcal{S} \subset \mathscr{M}$ uma superfície de Cauchy e $\nu \in \mathfrak{X}(\mathcal{S})$ um campo vetorial normal e unitário.

E sejam $\rho_{0}: \mathfrak{F}(\mathscr{M}) \rightarrow \mathfrak{F}(\mathcal{S})$ o operador restrição, $\rho_{0}(u)=\left.u\right|_{\mathcal{S}}$, e $\rho_{1}: \mathfrak{F}(\mathscr{M}) \rightarrow$ $\mathfrak{F}(\mathcal{S})$ o operador derivada na direção $\nu$ em $\mathcal{S}, \rho_{1}(u)=\partial_{\nu} u$, dada em coordenadas locais por: $\partial_{\nu} u=\left.\nu^{i}\left(\partial u / \partial x^{i}\right)\right|_{\mathcal{S}}$.

O problema de Cauchy para $P$ com os dados $\left(f, u_{0}, u_{1}\right), f \in \mathfrak{F}(\mathscr{M}), u_{0}, u_{1} \in$ $\mathfrak{F}(\mathcal{S})$, e $u \in \mathfrak{F}(\mathscr{M})$ é o sistema:

$$
\left\{\begin{array}{l}
P u=f \\
\rho_{0}(u)=u_{0} \\
\rho_{1}(u)=u_{1}
\end{array}\right.
$$

Um ponto central desta seção é indicar na direção do seguinte teorema:

Teorema 3.1. Existe uma única $u \in \mathfrak{F}(\mathscr{M})$ que satisfaz (8) e que ainda suppu $\subset J^{+}(K) \cup J^{-}(K)$ onde $K=\operatorname{supp}\left(u_{0}\right) \cup \operatorname{supp}\left(u_{1}\right) \cup \operatorname{supp}(f)$.

Por fim, operadores de Green (retardados e avançados), para o operador $P$, são operadores lineares

$$
G_{ \pm}: \mathfrak{F}_{0}(\mathscr{M}) \rightarrow \mathfrak{F}(\mathscr{M})
$$

que satisfazem:

$$
\text { (i) } P \circ G_{ \pm}=\mathbb{1},\left.\quad G_{ \pm} \circ P\right|_{\mathfrak{F}_{0}(\mathscr{M})}=\mathbb{1} ;
$$

(ii) $\operatorname{supp}\left(G_{ \pm} \phi\right) \subset J^{ \pm}(\operatorname{supp} \phi), \forall \phi \in \mathfrak{F}_{0}(\mathscr{M})$.

Mostraremos também que $G_{ \pm}$está ligado a soluções fundamentais de $P$. 


\subsubsection{Soluções Fundamentais}

Soluções Fundamentais em espaços-tempos de Minkowski Primeiro, acharemos uma solução fundamental de $\square$ em 0 num espaço-tempo de Minkowski $\left(\mathbb{R}^{n}, \eta\right)$, da qual poderemos construir soluções locais num espaçotempo mais geral.

Seja $\eta(x)=\eta_{\nu \mu} x_{\nu} x_{\mu}$, definimos as funções $R_{ \pm}^{\alpha}: \mathbb{R}^{n} \rightarrow \mathbb{R}$, para $\alpha \in \mathbb{C}$, $\Re(\alpha)>n$ :

$$
R_{ \pm}^{\alpha}(x)=\left\{\begin{array}{ll}
C(\alpha, n) \eta(x)^{(\alpha-n) / 2} & \text { se } x \in J^{ \pm}(0) \\
0 & \text { se } x \notin J^{ \pm}(0)
\end{array},\right.
$$

onde

$$
C(\alpha, n)=\frac{2^{1-\alpha} \pi^{(2-n) / 2}}{\Gamma(\alpha / 2-1) \Gamma((\alpha-n) / 2)},
$$

onde $\Gamma$ é a função Gamma de Euler, $\Gamma(z)=\int_{0}^{\infty} t^{z-1} e^{-t} d t, \Re(z)>1$, que sabemos que satisfaz $\Gamma(z)=z \Gamma(z-1)$.

Afirmamos que $R_{ \pm}^{\alpha}$ satisfaz a equação $\square R_{ \pm}^{\alpha+2}=R_{ \pm}^{\alpha}$, e para mostrar isso mostraremos que: (1) $\eta R_{ \pm}^{\alpha}=\alpha(\alpha-n+2) R_{ \pm}^{\alpha+2}$ e $(2)\left(\partial_{\mu} \eta\right) R_{ \pm}^{\alpha}=2 \alpha \partial_{\mu} R_{ \pm}^{\alpha+2}$. Caso (1) e (2) sejam verdades calculamos, para $\Re(\alpha) \geq n+2$ :

$$
\begin{gathered}
\partial_{\mu}^{2} R_{ \pm}^{\alpha+2}=\partial_{\mu} \partial^{\mu} R_{ \pm}^{\alpha+2}=\partial_{\mu}\left(\frac{1}{2 \alpha}\left(\partial_{\mu} \eta\right)\left(R_{ \pm}^{\alpha}\right)\right) \\
=\frac{1}{2 \alpha}\left(\partial_{\mu}^{2} \eta R_{ \pm}^{\alpha}+\partial_{\mu} \eta \partial_{\mu} R_{ \pm}^{\alpha}\right)=\frac{1}{2 \alpha}\left(\partial_{\mu}^{2} \eta R_{ \pm}^{\alpha}+\frac{1}{2(\alpha-2)}\left(\partial_{\mu} \eta\right)^{2} R_{ \pm}^{\alpha-2}\right),
\end{gathered}
$$

onde $\partial_{\mu} \eta(x)=2 \eta_{\mu \nu} x^{\nu}$ e $\partial_{\mu}^{2} \eta(x)=2 \eta_{\mu \nu} \eta^{\mu \nu}=2 n$. Então:

$$
\square R_{ \pm}^{\alpha+2}(x)=\frac{1}{2 \alpha}\left(2 n R_{ \pm}^{\alpha}(x)+\frac{2}{\alpha-2}\left(\eta_{\mu \nu} x^{\nu}\right)^{2} R_{ \pm}^{\alpha-2}(x)\right) .
$$

Mas, $\left(\eta_{\mu \nu} x^{\nu}\right)^{2}=\eta_{\mu \nu} x^{\nu} \eta^{\mu \lambda} x_{\lambda}=x^{\lambda} x_{\lambda}=\eta(x)$, portanto,

$$
\begin{array}{r}
\square R_{ \pm}^{\alpha+2}(x)=\frac{1}{\alpha}\left(n R_{ \pm}^{\alpha}(x)+\frac{1}{2-\alpha}\left(\eta R_{ \pm}^{\alpha-2}\right)(x)\right)= \\
=\frac{1}{\alpha}\left(n R_{ \pm}^{\alpha}(x)+(\alpha-n) R_{ \pm}^{\alpha}(x)\right)=R_{ \pm}^{\alpha}(x) .
\end{array}
$$

Mostremos, portanto, a validade de (1) e (2). Em regiões foras de $J^{ \pm}(x)$ as igualdades são triviais, tomemos, então, $R_{ \pm}^{\alpha}$ em $J^{ \pm}(x)$. Vejamos a igualdade (1): $\eta R_{ \pm}^{\alpha}=C(\alpha, n) \eta^{(\alpha-n) / 2+1}=C(\alpha, n) \eta^{(\alpha+2-n) / 2}$, mas, $R_{ \pm}^{\alpha+2}=C(\alpha+$ $2, n) \eta^{(\alpha 2-n) / 2}$, então, $\eta R_{ \pm}^{\alpha}=C(\alpha, n) / C(\alpha+2, n) R_{ \pm}^{\alpha+2}$. E:

$$
\frac{C(\alpha, n)}{C(\alpha+2, n)}=\frac{2^{1-\alpha} \pi^{(2-n) / 2}}{\Gamma(\alpha / 2-1) \Gamma((\alpha-n) / 2)} \frac{\Gamma((\alpha+2) / 2-1) \Gamma((\alpha+2-n) / 2)}{2^{1-\alpha-2} \pi^{(2-n) / 2}}
$$


$=2^{2} \frac{1}{\Gamma(\alpha / 2-1) \Gamma((\alpha-n) / 2)} \frac{\alpha}{2} \Gamma(\alpha / 2-1)\left(\frac{\alpha-n}{2}+1\right) \Gamma((\alpha-n) / 2)=\alpha(\alpha-n+2)$.

Isso prova (1). Vejamos agora (2):

$$
\begin{aligned}
& \partial_{\mu}\left(\eta \eta^{(\alpha-n) / 2}\right)=\left(\partial_{\mu} \eta\right) \eta^{(\alpha-n) / 2}+\eta \frac{\alpha-n}{2} \eta^{(\alpha-n) / 2-1} \partial_{\mu} \eta= \\
& \quad=\partial_{\mu} \eta\left(\eta^{(\alpha-n) / 2}+\frac{\alpha-n}{2} \eta^{(\alpha-n) / 2}\right)=\frac{\alpha-n+2}{2} \eta^{(\alpha-n) / 2} \partial_{\mu} \eta .
\end{aligned}
$$

Então,

$$
\begin{gathered}
\left(\partial_{\mu} \eta\right) R_{ \pm}^{\alpha}=C(\alpha, n) \eta^{(\alpha-n) / 2} \partial_{\mu} \eta=\frac{2 C(\alpha, n)}{\alpha+2-n} \partial_{\mu}\left(\eta \eta^{(\alpha-n) / 2}\right) \\
=2 \alpha \partial_{\mu}\left(C(\alpha+2, n) \eta^{(\alpha+2-n) / 2}\right)=2 \alpha \partial_{\mu} R_{ \pm}^{\alpha+2} .
\end{gathered}
$$

Onde usamos o resultado da conta anterior: $2 C(\alpha, n) /(\alpha+2-n)=2 \alpha C(\alpha+$ $2, n)$.

Devemos estender $\alpha \mapsto R_{ \pm}^{\alpha}$ para todo plano complexo. Como $n \geq 4 \mathrm{e}$ a função gamma é holomorfa em $\mathbb{C} \backslash\{0,-1,-2, \ldots\}, R_{ \pm}^{\alpha}$ é holomorfa em $\{\alpha: \Re(\alpha)>n\}$, e é isso que nos garante que a demonstração acima seja valida para $\Re \alpha>n$. Para $\Re \alpha>n-2$ definimos a função $\hat{R}_{ \pm}^{\alpha}=\square R_{ \pm}^{\alpha+2}$, e $\{\alpha: \Re \alpha>n-2\} \ni \alpha \mapsto \hat{R}_{ \pm}^{\alpha}$ é holomorfa, e $\hat{R}_{ \pm}^{\alpha}=R_{ \pm}^{\alpha}$ quando restrito à $\{\alpha: \Re \alpha>n\}$, de modo que $\hat{R}_{ \pm}^{\alpha}$ é uma extensão holomorfa de $R_{ \pm}^{\alpha}$. Repetindo esse processo construímos uma função holomorfa em $\{\alpha: \Re \alpha>n-4\}$, que coincide com $\alpha \mapsto \hat{R}_{ \pm}^{\alpha}$ em $\{\alpha: \Re \alpha>n-2\}$, e assim por diante, até obter uma função holomorfa em $\mathbb{C}{ }^{34}$

É dessa função que definimos a distribuição avançada e retardada de Riesz, identificando a função com sua distribuição, como dissemos que é possível fazer em seção anterior. A distribuição $R_{ \pm}^{\alpha}$ ainda satisfaz as propriedades que foram demonstradas para a sua função associada. Além disso, vemos claramente que $\operatorname{supp}\left(R_{ \pm}^{\alpha}\right) \subset J^{ \pm}(0)$. Resta mostrar que $R_{ \pm}^{0}=\delta_{0}$, e então conluiremos facilmente que $R_{ \pm}^{2}$ é uma solução fundamental (avançada ou retardada) em 0 de $\square$.

Soluções Fundamentais Locais Seja o cobrimento $\left\{\left(\mathscr{U}_{\lambda}, h_{\lambda}\right)\right\}$, e cada $\mathscr{U}_{\lambda}$ convexo. Tomamos $\mathscr{U} \subset \mathscr{M}$ uma vizinhança normal de um ponto $p$, que pertence a alguma carta local convexa, e seja $\mu_{p}=\sqrt{g_{p}}$. A distribuição (avançada caso o subscrito seja + e retardada caso seja -) de Riesz em $\mathscr{U}$ é a distribuição $R_{ \pm}^{(\mathscr{U}, \alpha, p)}$ dada localmente por

$$
R_{ \pm}^{(\mathscr{U}, \alpha, p)}(\phi)=R_{ \pm}^{\alpha}\left(\left(\mu_{p} \phi\right) \circ \exp _{p}\right), \quad \forall \phi \in \mathfrak{F}_{0}(\mathscr{U}) .
$$

\footnotetext{
${ }^{34} \mathrm{Em}$ casos realistas de $n=4$ esse processo pode ser esgotado na terceira etapa, já que, como veremos, importa saber a distribuição de Riesz (definida abaixo) em ao menos $\alpha=0$.
} 
Onde é claro que $\mu_{p}: \mathscr{U} \rightarrow \mathbb{R}$ atua apenas como escala, e, sendo $\exp _{p}^{-1}(\mathscr{U})=$ $\mathscr{U}^{\prime} \subset \mathbb{R}^{n}, \mathfrak{F}_{0}\left(\mathscr{U}^{\prime}\right) \ni \phi \circ \exp _{p}: \mathscr{U}^{\prime} \rightarrow \mathbb{R}$.

As distribuições de Riesz satisfazem as seguintes propriedades:

$$
\begin{aligned}
& \text { (1) } R_{ \pm}^{(\mathscr{U}, 0, p)}=\delta_{p} ;\left.\quad(2) \operatorname{supp}\left(R_{ \pm}^{(\mathscr{U}, \alpha, p)}\right) \subset J^{ \pm}(p)\right|_{\mathscr{U}} ; \\
& \text { (3) } \square R_{ \pm}^{(\mathscr{U}, \alpha+2, p)}=\left(\frac{\square \Gamma_{p}-2 n}{2 \alpha}+1\right) R_{ \pm}^{(\mathscr{U}, \alpha, p)}, \forall \alpha \neq 0 .
\end{aligned}
$$

Onde $\Gamma_{p}=\eta \circ \exp _{p}^{-1}$. Infelizmente $R_{ \pm}^{(\mathscr{U}, \alpha, p)}$ não são soluções fundamentais de $\square$ em $\mathscr{U}$, como bem mostra a propriedade (3). Mas a partir dessas distribuições tomaremos um Ansatz na forma de uma série, cujos coeficientes devem satisfazer uma equação diferencial para que o Ansatz seja solução de $P$.

O Ansatz é o seguinte:

$$
R_{ \pm}^{(\mathscr{U}, p)}=\sum_{k=0}^{\infty} V_{p}^{k} R_{ \pm}^{(\mathscr{U}, 2 k+2, p)}
$$

onde $p \mapsto V_{p}^{k}$ é suave, e se substituirmos essa equação em $P R_{ \pm}^{(\mathscr{U}, p)}=\delta_{p}$, obtemos as condições para os coeficientes:

$$
\nabla_{\operatorname{grad} \Gamma_{p}} V_{p}^{k}-\left(\frac{1}{2} \square \Gamma_{p}-n+2 k\right) V_{p}^{k}=2 k P V_{p}^{k-1} .
$$

Chamamos a sequencia de funções $\left(V_{. .}^{k}\right)_{k} \subset \mathfrak{F}(\mathscr{U} \times \mathscr{U})$, tal que $V_{p, p}^{0}=1$, e cujos elementos $V_{p}^{k}=V_{p}^{k}$. satisfazem a equação acima, uma sequencia de coeficientes de Hadamard para P. E claro, se construirmos o Ansatz (9) de modo que os coeficientes são coeficientes de Hadmard obteremos uma solução fundamental formal de $P$ em $p$.

A partir de (9) podemos construir uma solução exata, limitando, em um certo sentido, a série da solução formal. E essa limitação, chamemo-a de $\tilde{R}_{ \pm}^{(\mathscr{U}, p)}$, fornecem, para um dominio $\mathscr{U}$ que satisfaz algumas condições, distribuições de Riesz que se aproximão de uma solução fundamental de $P$. De forma mais precisa: $\mathscr{U}$ deve ser um conjunto cujo fecho $\overline{\mathscr{U}}$ é compacto, e deve estar contido em um subconjunto convexo $\mathscr{U}^{\prime}$ de $\mathscr{M}$. Então $\tilde{R}_{ \pm}^{(\mathscr{U}, p)}$ é uma distribuição em $\mathscr{U}$, suave em $p$, com $P_{2} \tilde{R}_{ \pm}^{(\mathscr{U}, p)}-\delta_{p}=K_{ \pm}(p,$.$) , onde$ $K_{ \pm} \in \mathfrak{F}(\overline{\mathscr{U}} \times \overline{\mathscr{U}})$ e onde o indice 2 em $P_{2}$ indica $P$ agindo na segunda componente dos coeficientes de Hadamard. Com a seguinte condição de suporte: $\operatorname{supp}\left(\tilde{R}_{ \pm}^{(\mathscr{U}, p)}\right) \subset J_{\mathscr{U}^{\prime}}^{ \pm}(p)$.

Identificando $K_{ \pm}(p,.) \in \mathfrak{F}(\overline{\mathscr{U}})$ com sua correspondente distribuição

$$
\mathfrak{F}_{0}(\overline{\mathscr{U}}) \ni \phi \mapsto \int_{\overline{\mathscr{U}}} K_{ \pm}(p, y) \phi(y) d V
$$


Reescrevemos $P_{2} \tilde{R}_{ \pm}^{(\mathscr{U}, p)}-\delta_{p}=K_{ \pm}(p,.) \operatorname{como}\left(P_{2} \tilde{R}_{ \pm}^{(\mathscr{U}, .)}\right) \phi=\left(\mathbb{1}+K_{ \pm}\right) \phi$, e com algumas restrições sobre a norma de $K_{ \pm} \in \mathfrak{F}(\overline{\mathscr{U}} \times \overline{\mathscr{U}})$ podemos inverter $\left(\mathbb{1}+K_{ \pm}\right)$. Definindo $f_{\phi} \in \mathfrak{F}(\overline{\mathscr{U}})$ como a função $p \mapsto \tilde{R}_{ \pm}^{(\mathscr{U}, p)}(\phi)$, a solução fundamental de $P$ é:

$$
F_{\mathscr{U}, p}^{ \pm}(\phi)=\left(\mathbb{1}+K_{ \pm}\right)^{-1} f_{\phi}(p) .
$$

Com esses resultados pode-se obter o seguinte corolário ([3]): Sejam o operador d'Alembertiano $P$ e o espaço-tempo $(\mathscr{M}, g)$, então, todo ponto $p$ de $\mathscr{M}$ possui uma vizinhança causal $\mathscr{U}$ cujo fecho é compacto, tal que existem soluções fundamentais (avançadas e retardadas) $F_{\mathscr{U}, p}^{ \pm}$, para $P$ em $\mathscr{U}$, suaves em $p$, que satisfazem a condição de $\operatorname{suporte} \operatorname{supp}\left(F_{\mathscr{U}, p}^{ \pm}\right) \subset J_{\mathscr{U}}^{ \pm}(p)$.

\subsubsection{O Problema de Cauchy e Operadores de Green}

Dada uma solução fundamental $F_{\mathscr{U}, p}^{ \pm}$de $P$, avançada e retardada (i.e., tal que $\left.\operatorname{supp}\left(F_{\mathscr{U}, p}^{ \pm}\right) \subset J_{\mathscr{U}}^{ \pm}(p)\right)$, a distribuição $u_{ \pm}$dada por, para cada $f \in \mathfrak{F}(\mathscr{U})$ :

$$
u_{ \pm}(\phi)=\int_{\mathscr{U}} f(x) F_{\mathscr{U}, x}^{ \pm}(\phi) d V, \quad \forall \phi \in \mathfrak{F}_{0}(\mathscr{U})
$$

é uma solução de $P u_{ \pm}=f$ no sentido distribucional,e ainda vale que $\operatorname{supp}\left(u_{ \pm}\right) \subset$ $J_{\mathscr{U}}^{ \pm}(\operatorname{supp} f)$.

É elementar que $u_{ \pm}$é solução de $P u_{ \pm}=f$ tendo em vista as contas feitas em seções anteriores (da motivação da definição de soluções fundamentais). Vejamos a condição do suporte:

Suponha que $\phi \in \mathfrak{F}_{0}(\mathscr{U})$ seja tal que $u_{ \pm}(\phi) \neq 0$, então, deve haver um $x \in \mathscr{U}$ tal que $f(x) F_{\mathscr{U}, x}^{ \pm}(\phi) \neq 0$, ou seja, $f(x) \neq 0$ e $F_{\mathscr{U}, x}^{ \pm}(\phi) \neq 0$. Portanto, $x \in \operatorname{supp} f \operatorname{exupp} \phi \cap \operatorname{supp}\left(F_{\mathscr{U}, x}^{ \pm}\right) \neq \emptyset$, mas $\operatorname{como} \operatorname{supp}\left(F_{\mathscr{U}, x}^{ \pm}\right) \subset J_{\mathscr{U}}^{ \pm}(x)$, $\operatorname{supp} \phi \cap J_{\mathscr{U}}^{ \pm}(x) \neq \emptyset$.

Se pensarmos, de forma rudimentar, como estão as posições desses conjuntos, concluiremos que podemos escrever: $x \in J_{\mathscr{U}}^{\mp}(\operatorname{supp} \phi)$. Então, $\operatorname{supp} f \cap$ $J_{\mathscr{U}}^{\mp}(\operatorname{supp} \phi) \neq \emptyset$, e, novamente, $\operatorname{supp} \phi \cap J_{\mathscr{U}}^{ \pm}(\operatorname{supp} f) \neq \emptyset$. Portanto, $\operatorname{supp} u_{ \pm} \subset$ $J_{\mathscr{U}}^{ \pm}(\operatorname{supp} f)$.

Com esses resultados, e seguindo essencialmente os mesmos passos que foram tomados nesta e na seção anterior, a saber, tomar um Ansatz formal (mas, neste caso, tomando uma solução em $\mathbb{R} \times(\mathcal{S} \cap \mathscr{U})$, que sempre pode ser feito, já que $\mathcal{S} \cap \mathscr{U}$ é uma superfície de Cauchy em $\mathscr{U}$, de modo que podemos identificar os dois através de uma isometria), identificar seus coeficientes de Hadamard, limitar a série, etc., é possível mostrar que há uma única solução 
para o problema de Cauchy local para $P$, que tem a seguinte formulação: Seja $\mathcal{S}$ uma hipersuperficie suave tipo-tempo, e $\nu \in \mathfrak{X}(\mathcal{S})$ um campo vetorial normal e únitario. Seja $\mathscr{U} \in \mathscr{M}$ um aberto causal com fecho compacto tal que $\mathcal{S} \cap \mathscr{U}$ seja uma superficie de Cauchy de $\mathscr{U}$. Sendo $\rho_{0}$ e $\rho_{1}$ a restrição à $\mathcal{S} \cap \mathscr{U}$ e a derivada normal em $\mathcal{S} \cap \mathscr{U}$, respectivamente, o problema de Cauchy com os dados $\left(f, u_{0}, u_{1}\right) \in \mathfrak{F}(\mathscr{U}) \oplus \mathfrak{F}(\mathcal{S} \cap \mathscr{U}) \oplus \mathfrak{F}(\mathcal{S} \cap \mathscr{U})$, é o sistema:

$$
\left\{\begin{array}{l}
P u=f \\
\rho_{0}(u)=u_{0} \\
\rho_{1}(u)=u_{1}
\end{array}\right.
$$

com $u \in \mathfrak{F}(\mathscr{U})$ com suporte $\operatorname{supp} u \subset J_{\mathscr{U}}^{+}(K) \cup J_{\mathscr{U}}^{-}(K)$ onde $K=\operatorname{supp}\left(u_{0}\right) \cup$ $\operatorname{supp}\left(u_{1}\right) \cup \operatorname{supp}(f)$.

Soluções globais de (8) são formadas tomando varias soluções locais. Identificase o espaço globalmente hiperbólico com o espaço-tempo $\mathbb{R} \times \mathcal{S}$, e então construímos soluções em $(-\varepsilon, \varepsilon) \times \mathcal{S}$. Novamente, os detalhes desta construção, bem como a prova da unicidade dessas soluções, estão descritas com todas as nuances e detalhes em [3].

Com soluções de (8) em mãos é possível construir soluções fundamentais globais, essas soluções servem para fazer conexão entre operadores de Green e soluções do problema de Cauchy. O conteúdo do teorema que nos garante soluções fundamentais globais é o seguinte:

Teorema 3.2. Sejam P um operador d'Alembertiano e $\mathscr{M}$ um espaço-tempo globalmente hiperbólico, então, existe, para todo ponto $x \in \mathscr{M}$, uma única solução fundamental $F^{+}$e uma única $F^{-}$, tal que, $\operatorname{supp}\left(F_{x}^{ \pm}\right) \subset J^{ \pm}(x)$ e tal que $\forall \phi \in \mathfrak{F}_{0}(\mathscr{M}), f_{\phi}^{ \pm}(x):=F_{x}^{ \pm}(\phi)$ é suave e $P^{*}\left(f_{\phi}^{ \pm}\right)=\phi$.

Lembrando que como estamos interessados em $P=\square+m^{2}, P^{*}=P$.

A prova dessa proposição consiste em dividir o espaço-tempo em foliações por superfícies de Cauchy, $\{t\} \times \mathcal{S} \ni x$, achar soluções globais $\chi_{\phi}$ nessas superfícies e definir $F_{x}^{ \pm}(\phi)=\chi_{\phi}(x)$.

Os operadores de Green $G_{ \pm}: \mathfrak{F}_{0}(\mathscr{M}) \rightarrow \mathfrak{F}(\mathscr{M})$, como definidos em seção anterior, têm uma relação simples com as soluções fundamentais de $P^{*}$. Consideremos $P=\square+m^{2}$, e seja $F^{ \pm}$as soluções fundamentais garantidas pelo teorema (3.2), tomando $P=\square+m^{2}$. Então, $G_{ \pm}$é dado, $\forall \phi \in \mathfrak{F}_{0}(\mathscr{M})$ por

$$
G_{\mp}(\phi)=f_{\phi}^{ \pm} .
$$

Ou seja, $\left(G_{\mp}(\phi)\right)(x)=F_{x}^{ \pm}(\phi)$. Que $G_{ \pm}$existe e são únicos segue do teorema que garante que há soluções fundamentais avançadas e retardadas únicas, e as propriedades de $G_{ \pm}$seguem dos predicados das soluções fundamentais. 
Vejamos: $\left(P \circ G_{ \pm}\right)(\phi)=P\left(G_{ \pm} \phi\right)=P\left(f_{\phi}^{\mp}\right)=\phi$, então $P \circ G_{ \pm}=\mathbb{1}$. Por outro lado, $\phi \mapsto f_{\phi}^{ \pm}(x)$ é uma distribuição, então, $\left(\left.G_{ \pm} \circ P\right|_{\mathfrak{F}_{0}(\mathscr{M})}\right)(\phi)(x)=$ $\left(G_{ \pm}\left(\left.P\right|_{\mathfrak{F}_{0}(\mathscr{M})} \phi\right)\right)(x)=P^{*} F_{x}^{\mp}(\phi)=\phi(x)$, de modo que $\left.G_{ \pm} \circ P\right|_{\mathfrak{F}_{0}(\mathscr{M})}=\mathbb{1}$.

A condição sobre o suporte de $G_{ \pm}$é demonstrada de forma análoga à demonstração da condição de suporte para soluções locais de $P$ : considere $\phi \in$ $\mathfrak{F}_{0}(\mathscr{M})$ tal que $\left(G_{ \pm}(\phi)\right)(x) \neq 0$, então, $F_{x}^{\mp}(\phi) \neq 0$, então, $x \in \operatorname{supp}\left(f_{\phi}^{\mp}\right)=$ $\operatorname{supp}\left(G_{ \pm}(\phi)\right)$ e $\operatorname{supp}(\phi) \cap \operatorname{supp}\left(F_{x}^{\mp}\right) \neq \emptyset . \quad \mathrm{E} \operatorname{como} \operatorname{supp}\left(F_{x}^{\mp}\right) \subset J^{\mp}(x)$, $x \in J^{ \pm}(\operatorname{supp}(\phi))$. Ou seja, mostramos que todo $x \in \operatorname{supp}\left(G_{ \pm}(\phi)\right)$ está em $J^{ \pm}(\operatorname{supp}(\phi))$, como requer a condição que define operadores de Green.

O modo como os operadores de Green - que são de fundamental importância para a quantização de campos em espaços-tempos globalmente hiperbólicos - são apresentados aqui segue o caminho traçado por Bär, Ginoux e Pfäffle, que de fato parece ser um caminho circular: achamos soluções fundamentais em espaços-tempos de Minkowski, e então soluções fundamentais locais para espaços-tempos mais gerais, das quais obtemos soluções locais do problema de Cauchy e com essas soluções construimos soluções globais. Dessas soluções globais voltamos às soluções fundamentais (globais). De fato há, aparentemente, um caminho alternativo, como apresentado, por exemplo, no artigo de 1980 de Dimock [13]: são consideradas soluções fundamentais globais de $\square+m^{2}$ em espaços-tempos globalmente hiperbólicos, das quais constrói-se operadores de Green, e destes as soluções globais do problema de Cauchy são derivadas.

\subsubsection{Propriedades das Soluções}

Fundamental para a quantização dos campos que satisfazem as relações CCR são os operadores de Green do operador $P=\square+m^{2}$, que podem ser entendidos grosso modo como o inverso de $P$. E veremos como através dos operadores de Green podemos escrever a solução do problema de Cauchy que nos interessa, em termos das condições iniciais, de modo que os operadores de Green carregam em si o conteúdo da equação de onda.

Primeiro, observamos que podemos estender os operadores de Green $G_{ \pm}$: $\mathfrak{F}_{0}(\mathscr{M}) \rightarrow \mathfrak{F}(\mathscr{M})$ a operadores $G_{ \pm}^{*}: \mathfrak{E}^{\prime}(\mathscr{M}) \rightarrow \mathfrak{D}^{\prime}(\mathscr{M})^{35}$ que agem em distribuições, através da mesma técnica que foi usada para estender operadores diferenciais para distribuições, ou seja, através do adjunto formal. Afirmamos que o adjunto formal do operador $G_{ \pm}$é o operador $G_{\mp}^{*}$ que é solução de

\footnotetext{
${ }^{35}$ Lembramos que $\mathfrak{D}^{\prime}(\mathscr{M})$ é o conjunto das distribuições em $\mathscr{M}$ e $\mathfrak{E}^{\prime}(\mathscr{M})$ é o conjunto das distribuições em $\mathscr{M}$ com suporte compacto.
} 
$P^{*}$. Em particular para $P=\square+m^{2}, G_{ \pm}^{*}=G_{\mp}$. A prova é a seguinte:

$$
\begin{aligned}
& \int u(x)\left(G_{ \pm} \phi\right)(x) d V=\int(\overbrace{P^{*} G_{\mp}^{*}}^{=\mathbb{1}} u)(x)\left(G_{ \pm} \phi\right)(x) d V= \\
& =\int\left(G_{\mp}^{*} u\right)(x)(\underbrace{P G_{ \pm}}_{=\mathbb{1}} \phi)(x) d V=\int\left(G_{\mp}^{*} u\right)(x) \phi(x) d V .
\end{aligned}
$$

Então, se definirmos $G=G_{+}-G_{-}$seu adjunto formal é $G^{*}=-G$, ou seja, $(G u)(\phi)=-u(G \phi)$.

Da mesma forma, podemos achar os adjuntos formais dos operadores de restrição à superfície de Cauchy $\mathcal{S}, \rho_{0}: \mathfrak{F}(\mathscr{M}) \rightarrow \mathfrak{F}(\mathcal{S})$, e o operador derivada normal em $\mathcal{S}, \rho_{1}: \mathfrak{F}(\mathscr{M}) \rightarrow \mathfrak{F}(\mathcal{S})$, e os chamaremos de $\rho_{i}^{\prime}: \mathfrak{E}^{\prime}(\mathcal{S}) \rightarrow \mathfrak{E}^{\prime}(\mathscr{M})$, $i=0,1$, temos então a composição $G \circ \rho_{i}^{\prime}=G \rho_{i}^{\prime}: \mathfrak{E}^{\prime}(\mathcal{S}) \rightarrow \mathfrak{D}^{\prime}(\mathscr{M})$. E, claro, podemos restringir distribuições à funções pela seguinte aplicação $\mathfrak{F}_{0}(\mathcal{S}) \ni$ $\phi \mapsto \int_{\mathcal{S}} \phi(x) u(x) d V_{s}{ }^{36}$, onde $d V_{s}$ é o elemento de volume induzido em $\mathcal{S}$, de modo que podemos pensar $G \rho_{i}^{\prime}: \mathfrak{F}_{0}(\mathcal{S}) \rightarrow \mathfrak{F}(\mathscr{M})$ como operadores entre funções.

Dessas definições segue o seguinte teorema:

Teorema $3.3([13])$. A solução $u \in \mathfrak{F}(\mathscr{M})$ do problema de Cauchy do operador $\square+m^{2}$ com os dados $\left(0, u_{0}, u_{1}\right)$ pode ser escrita da seguinte forma:

$$
u=\left(G \rho_{0}^{\prime}\right) u_{1}-\left(G \rho_{1}^{\prime}\right) u_{0} .
$$

Demonstração. Na prova usaremos a forma integral da solução do problema de Cauchy em questão:

$$
\int_{\mathscr{M}} u f d V=\int_{\mathcal{S}}\left(u_{0} \rho_{1}(G f)-u_{1} \rho_{0}(G f)\right) d V_{s}
$$

que é provada se usarmos a identidade de Green, $\int_{D}\left[u\left(\square+m^{2}\right) v-v(\square+\right.$ $\left.\left.m^{2}\right) u\right] d V=\int_{\partial D}(u \partial v / \partial n-v \partial u / \partial n) d S$ e dividirmos o espaço-tempo $\mathscr{M}$ entre $D^{-}=J^{-}(\mathcal{S}) / \mathcal{S}$ e $D^{+}=J^{+}(\mathcal{S}) / \mathcal{S}$. Nessas situações $\partial D^{ \pm}=\mathcal{S}$.

No primeiro caso tomamos $u$ como solução de $\left(\square+m^{2}\right)$ e $v=G_{+} f$, e $\rho_{1}=$ $\partial / \partial n$ e no segundo caso tomamos $u$ de novo como solução de $\left(\square+m^{2}\right)$ mas $v=G_{-} f$ e $\rho_{1}=-\partial / \partial n$. E como $\left(\square+m^{2}\right) G_{ \pm}=\mathbb{1}$ temos as seguintes igualdades:

$$
\int_{D^{-}} u f d V=\int_{\mathcal{S}}\left(u_{0} \rho_{1}\left(G_{+} f\right)-u_{1} \rho_{0}\left(G_{+} f\right)\right) d V_{s}
$$

\footnotetext{
${ }^{36}$ Chamaremos a aplicação $\phi, \psi \mapsto \int \phi \psi$ de $\langle\phi, \psi\rangle$.
} 


$$
\int_{D^{+}} u f d V=-\int_{\mathcal{S}}\left(u_{0} \rho_{1}\left(G_{-} f\right)-u_{1} \rho_{0}\left(G_{-} f\right)\right) d V_{s}
$$

cuja soma nos fornece (11), e se pensarmos em (11) em termos de distribuições teremos as seguintes igualdades: $\int_{\mathscr{M}} u f d V=\langle u, f\rangle$,

$$
\int_{\mathcal{S}} u_{0} \rho_{1}(G f) d V_{s}=\left\langle u_{0}, \rho_{1} G f\right\rangle=-\left\langle G \rho_{1}^{\prime} u_{0}, f\right\rangle
$$

E analogamente $\int_{\mathcal{S}} u_{1} \rho_{0}(G f) d V_{s}=-\left\langle G \rho_{0}^{\prime} u_{1}, f\right\rangle$, de modo que $u=-G \rho_{1}^{\prime} u_{0}+$ $G \rho_{0}^{\prime} u_{1}$, em sentido distribucional. A igualdade no sentido funcional segue da observação de que se nos restringirmos aos casos $u_{1}=0$ ou $u_{0}=0$ os operadores $G \rho_{i}$ são entre funções.

Observação: A igualdade (11) será fundamental para a quantização dos campos bosônicos, e a escrevemos em forma distribucional:

$$
\langle u, f\rangle=\left\langle u_{0}, \rho_{1} G f\right\rangle-\left\langle u_{1}, \rho_{0} G f\right\rangle,
$$

onde, do lado esquerdo $\langle f, g\rangle=\int_{\mathscr{M}} f g d V$, enquanto que no lado direito $\langle f, g\rangle=\int_{\mathcal{S}} f g d V_{s}$.

Da forma (10) da solução obtemos as seguintes igualdades ([13]) que nos serão úteis: ao aplicar $\rho_{0}$ em (10): $u_{0}=\rho_{0} G \rho_{0}^{\prime} u_{1}-\rho_{0} G \rho_{1}^{\prime} u_{0}$, portanto,

$$
\rho_{0} G \rho_{0}^{\prime}=0, \quad \rho_{0} G \rho_{1}^{\prime} u_{0}=-\mathbb{1} .
$$

E aplicando $\rho_{1}: u_{1}=\rho_{1} G \rho_{0}^{\prime} u_{1}-\rho_{1} G \rho_{1}^{\prime} u_{0}$, portanto,

$$
\rho_{1} G \rho_{0}^{\prime}=\mathbb{1}, \quad \rho_{1} G \rho_{1}^{\prime}=0 .
$$

E por fim, se escrevermos $u_{i}=\rho_{i} u$ e $u=G f$, obteremos a seguinte igualdade

$$
G=G \rho_{0}^{\prime} \rho_{1} G-G \rho_{1}^{\prime} \rho_{0} G .
$$

Exemplo Darei agora um exemplo de função de Green de $\square+m^{2}$ em um espaço-tempo de Minkowski $\left(\mathbb{R}^{4}, \eta\right)$, com $\eta$ com signatura $(1,-1,-1,-1)$.

A determinação da função de Green de $\square+m^{2}$ no espaço-tempo de Minkowski é um problema bem conhecido: começa-se com o Ansatz $\phi=\int G(x-$ y) $f(y) d^{4} y, f(x)=\int f(y) \delta(x-y) d^{4} y$, escreve-se $G$ em termos de sua transformada de Fourier, etc. Deste processo calcula-se:

$$
\left(G_{ \pm}(f)\right)(x)=\frac{1}{(2 \pi)^{2}} \int_{C_{ \pm} \times \mathbb{R}^{3}} \frac{e^{i p x} \hat{f}(p)}{p^{2}+m^{2}} d^{4} p
$$


onde $p x=p_{\mu} x^{\mu}, p^{2}=p_{\mu} p^{\mu}$, e $\hat{f}$ é a transformada de Fourier de $f$. É claro que $\left(G_{ \pm}(f)\right)(x)$ é solução de $\left(\square+m^{2}\right) \phi=f$, já que importa apenas a ação (de $\square_{x}$ ) em $e^{i p x}$ quando aplicamos $\square_{x}$ em $\left(G_{ \pm}(f)\right)(x)$, e dessa ação obtemos $\square_{x} e^{i p x}=p^{2} e^{i p x}$, então, $\left(\square_{x}+m^{2}\right)\left(G_{ \pm}(f)\right)(x)=(2 \pi)^{-2} \int_{C_{ \pm} \times \mathbb{R}^{3}} e^{i p x} \hat{f}(p) d p$, e podemos tomar $C_{ \pm}=\mathbb{R}$, de modo que a integral acima é a transformada de fourier inversa de $\hat{f}$, ou seja, é $f$.

Podemos integrar em $p_{0}$ e obter uma forma explícita de $G_{ \pm}$, sem que a diferença entre as funções de Green retardada e avançada fiquem escondidas nas curvas (a princípio um tanto arbitrárias - por isso já começo com a função de Green sem antes falar de soluções fundamentais) $C_{ \pm}$. Escrevemos $p^{2}+m^{2}=p_{0}^{2}-\mathbf{p}^{2}+m^{2}=p_{0}-\omega_{p}^{2}=\left(p_{0}-\omega_{p}\right)\left(p_{0}+\omega_{p}\right)$, então,

$$
\left(G_{ \pm}(f)\right)(x)=\frac{1}{(2 \pi)^{2}} \int_{\mathbb{R}^{3}} e^{-i \mathbf{p} \cdot \mathbf{x}}(\underbrace{\left.\int_{C_{ \pm}} \frac{e^{i p_{0} x^{0}} \hat{f}\left(p_{0}, \mathbf{p}\right)}{\left(p_{0}-\omega_{p}\right)\left(p_{0}+\omega_{p}\right)} d p_{0}\right)}_{\mathcal{I}_{ \pm}} d^{3} p .
$$

Tomamos a curva $C_{+}$como a curva em sentido anti-horário em torno de $\omega_{p}$, e a curva $C_{-}$como a curva em sentido horário em torno de $-\omega_{p}$. Portanto:

$$
\begin{gathered}
\mathcal{I}_{+}=2 \pi i \operatorname{Res}\left(\frac{e^{i p_{0} x^{0}} \hat{f}\left(p_{0}, \mathbf{p}\right)}{\left(p_{0}-\omega_{p}\right)\left(p_{0}+\omega_{p}\right)}\right)_{p_{0}=\omega_{p}}=2 \pi i \frac{e^{i \omega_{p} x^{0}} \hat{f}\left(\omega_{p}, \mathbf{p}\right)}{2 \omega_{p}}, \\
\mathcal{I}_{-}=-2 \pi i \operatorname{Res}\left(\frac{e^{i p_{0} x^{0}} \hat{f}\left(p_{0}, \mathbf{p}\right)}{\left(p_{0}-\omega_{p}\right)\left(p_{0}+\omega_{p}\right)}\right)_{p_{0}=-\omega_{p}}=2 \pi i \frac{e^{-i \omega_{p} x^{0}} \hat{f}\left(-\omega_{p}, \mathbf{p}\right)}{2 \omega_{p}} .
\end{gathered}
$$

Então,

$$
\left(G_{+} f\right)(x)=\frac{i}{2 \pi} \int_{\mathbb{R}^{3}} \frac{1}{2 \omega_{p}} e^{i \omega_{p} x^{0}-i \mathbf{p} \cdot \mathbf{x}} \hat{f}\left(\omega_{p}, \mathbf{p}\right) d^{3} p=\left.\frac{i}{2 \pi} \int_{\mathbb{R}^{3}} \frac{e^{i p x}}{2 \omega_{p}} \hat{f}(p)\right|_{p_{0}=\omega_{p}} d^{3} p,
$$

$\mathrm{e}$

$$
\left(G_{-} f\right)(x)=\frac{i}{2 \pi} \int_{\mathbb{R}^{3}} \frac{1}{2 \omega_{p}} e^{-i \omega_{p} x^{0}-i \mathbf{p} \cdot \mathbf{x}} \hat{f}\left(-\omega_{p}, \mathbf{p}\right) d^{3} p=\left.\frac{i}{2 \pi} \int_{\mathbb{R}^{3}} \frac{e^{-i p x}}{2 \omega_{p}} \hat{f}(-p)\right|_{p_{0}=\omega_{p}} d^{3} p .
$$

Na integral de $\left(G_{-} f\right)(x)$ foi feita a mudança $p_{i} \mapsto-p_{i}$. Nesse caso o sinal de $d p_{i}$ compensa a mudança nos limites da integral, e $\omega_{-p}=\omega_{p}$. Calculamos $G=G_{+}-G_{-}$como sendo:

$$
(G f)(x)=\left.\frac{i}{2 \pi} \int_{\mathbb{R}^{3}} \frac{1}{2 \omega_{p}}\left(e^{i p x} \hat{f}(p)-e^{-i p x} \hat{f}(-p)\right)\right|_{p_{0}=\omega_{p}} d^{3} p .
$$


Apenas das propriedades do operador de Green é fácil vermos que $G f$, para qualquer $f \in \mathfrak{F}(\mathscr{M})$ é uma solução da equação de Klein-Gordon homogênea, e, portanto, há valores iniciais $\rho_{i}(G f)$ e, então, $G f$ é uma solução de um problema de Cauchy da equação de Klein-Gordon homogênea com os dados $\left(\rho_{0}(G f), \rho_{1}(G f)\right)$. Mas importa também saber se dado um problema de Cauchy com os dados $\left(u_{0}, u_{1}\right)$ a solução $G f$ é solução deste problema de Cauchy. O seguinte teorema impõe restrições à $f$ para que isso seja verdade:

Teorema 3.4. [13] Seja u uma solução do problema de Cauchy da equação de Klein-Gordon homogênea com $\rho_{i}(u)$ com suporte em um compacto $N \subset \mathcal{S}$. Então, para qualquer vizinhança aberta $\mathcal{O}$ de $N$ há uma $f \in \mathfrak{F}_{0}(\mathscr{M})$ com suppf $\subset \mathcal{O}$ e $u=G f$.

\section{Quantização}




\subsection{Axiomas de Haag-Kastler Generalizados}

Chamaremos de axiomas de Haag-Kastler generalizados os axiomas sugeridos por Dimock em [13], cuja ideia essencial é associar a cada região do espaçotempo uma $\mathrm{C}^{*}$-álgebra, que descreve os operadores locais, ou seja, a relação

$$
\mathcal{O} \mapsto \mathscr{A}(\mathcal{O})
$$

descreve os operadores na região $\mathcal{O}$. Os axiomas generalizados são:

(1) As regiões para as quais a relação (14) está definida são conjuntos abertos e limitados em $\mathscr{M}$. A coleção desses conjuntos forma um conjunto de índices (com a ordem sendo inclusão) com ortogonalidade, com ortogonalidade definida por: $\mathcal{O}_{1} \perp \mathcal{O}_{2} \Leftrightarrow \mathcal{O}_{1}$ e $\mathcal{O}_{2}$ possuem uma separação tipo-espaço (i.e., não há curvas causais unindo os pontos de $\mathcal{O}_{1}$ com os pontos de $\mathcal{O}_{2}$ ).

E a rede de $\mathrm{C}^{*}$-álgebras $\{\mathscr{A}(\mathcal{O})\}$ satisfaz a estrutura que define uma álgebra quasi-local $\mathscr{A}$. Temos, portanto, uma álgebra quasi-local $\mathscr{A}=\overline{\bigcup_{\mathcal{O}} \mathscr{A}(\mathcal{O})}$.

(2) Para toda isometria $\kappa$ entre $(\mathscr{M}, g)$ e $(\hat{\mathscr{M}}, \hat{g})$ há um isomorfismo entre as álgebras quasi-locais nesses espaços-tempos: $\alpha_{\kappa}: \mathscr{A} \rightarrow \hat{\mathscr{A}}$ tal que $\alpha_{\kappa}(\mathscr{A}(\mathcal{O}))=\hat{\mathscr{A}}(\kappa(\mathcal{O})), \alpha_{1}=1$ e $\alpha_{\kappa_{1}} \circ \alpha_{\kappa_{2}}=\alpha_{\kappa_{1} \circ \kappa_{2}}$.

(3) $\mathscr{A}$ é primitivo.

(4) Se $\mathcal{O}_{1}$ depender causalmente de $\mathcal{O}_{2}$, então, $\mathscr{A}\left(\mathcal{O}_{1}\right) \subseteq \mathscr{A}\left(\mathcal{O}_{2}\right)$

Comentários: Os axiomas (1) e (3) são generalizações imediatas dos respectivos axiomas de Haag-Kastler. O axioma (2) é uma generalização do axioma da invariância de Poincaré, e, de fato, este é recuperado se nos restringirmos à situação $(\mathscr{M}, g)=(\hat{\mathscr{M}}, \hat{g})$ ( $\alpha$ se torna, então, um automorfismo) e à espaços-tempos de Minkowski, então as isometrias formam o grupo de Poincaré. O axioma (4) pretende apenas expressar o fato que o futuro depende do passado; falta, no entanto, definir o que significa uma região depender causalmente de outra: diremos que uma região $\mathcal{O}_{1}$ depende causalmente de $\mathcal{O}_{2}$ se para toda curva causal inextensível que intersectar $\mathcal{O}_{1}$ também intersectar $\mathcal{O}_{2}$ em $\mathcal{S} \cap \mathcal{O}_{2} \neq \emptyset$, onde $\mathcal{S}$ é uma superfície de Cauchy.

\subsection{Quantização de Campos Escalares Livres}

O objetivo desta seção é quantizar campos escalares livres que satisfazem as relações canonicas de comutação, para isso o que vamos fazer é especificar a forma simplética não-degenerada que torna a álgebra de CCR-Weyl com um 
conteúdo físico, mas não sem uma explicação de sua motivação antes. Esta seção é baseada no artigo [13.

Motivação $\mathrm{O}$ que faremos é definir em que sentido álgebras CCR são realizadas ${ }^{37}$ sobre uma superfície de Cauchy. Uma realização das álgebras CCR em uma superfície de Cauchy $\mathcal{S}$ é a terna $\left\{(\mathscr{H}, \theta(f), \pi(f)): f \in \mathfrak{F}_{0}(\mathcal{S})\right\}$, onde $\mathscr{H}$ é um espaço de Hilbert complexo e $\theta(f)$ e $\pi(f)$ são operadores simétricos lineares que satisfazem:

$$
[\theta(f), \pi(g)]=i\langle f, g\rangle, \quad[\theta(f), \theta(g)]=[\pi(f), \pi(g)]=0,
$$

onde $\langle f, g\rangle=\int_{\mathcal{S}} f g d V_{s}$. Então, o operador em sentido distribucional:

$$
\phi=G \rho_{0}^{\prime} \pi-G \rho_{1}^{\prime} \theta
$$

é solução da equação de Klein-Gordon: $\left(\square+m^{2}\right) \phi=0$. O que isso significa é que temos um operador tomando valores em distribuições que é solução de uma equação de campo em um espaço-tempo globalmente hiperbólico, e dessa solução abstrairemos os elementos que importam para a quantização num espaço-tempo curvo, a saber, seu conteúdo algébrico.

O operador $\phi$ pode ser escrito, em sentido funcional como (basta lembrar que o adjunto-formal de $G$ é $-G$ ):

$$
\phi(f)=\theta\left(\rho_{1} G f\right)-\pi\left(\rho_{0} G f\right),
$$

para toda $f \in \mathfrak{F}(\mathscr{M})$, e, claro, $\rho_{i} G: \mathfrak{F}_{0}(\mathscr{M}) \rightarrow \mathfrak{F}(\mathcal{S})$, satisfaz a equação de Klein-Gordon: $\phi\left(\left(\square+m^{2}\right) f\right)=0$, já que $G\left(\square+m^{2}\right)=G_{+}\left(\square+m^{2}\right)-G_{-}(\square+$ $\left.m^{2}\right)=0$.

E ainda, $[\phi(f), \phi(g)]=-i\langle f, G g\rangle$. A prova é a seguinte:

$$
\begin{gathered}
{[\phi(f), \phi(g)]=\left[\theta\left(\rho_{1} G f\right)-\pi\left(\rho_{0} G f\right), \theta\left(\rho_{1} G g\right)-\pi\left(\rho_{0} G g\right)\right]} \\
=-\left(\left[\pi\left(\rho_{0} G f\right), \theta\left(\rho_{1} G g\right)\right]+\left[\theta\left(\rho_{1} G f\right), \pi\left(\rho_{0} G g\right)\right]\right) \\
=-i\left(\left\langle g, G \rho_{1}^{\prime} \rho_{0} G f\right\rangle-\left\langle G \rho_{0}^{\prime} \rho_{1} G f, g\right\rangle\right)=-i\langle g, \underbrace{\left(G \rho_{1}^{\prime} \rho_{0} G-G \rho_{0}^{\prime} \rho_{1} G\right)}_{=-G} f\rangle \\
=-i\langle f, G g\rangle .
\end{gathered}
$$

Com isso tomamos a exponencial como de costume: $W(f, g)=e^{i(\theta(f)-\pi(g))}$, e esses operadores satisfazem a seguinte relação:

$$
W\left(f_{1}, g_{1}\right) W\left(f_{2}, g_{2}\right)=e^{-i\left(\left\langle f_{1}, g_{2}\right\rangle-\left\langle f_{2}, g_{1}\right\rangle\right) / 2} W\left(f_{1}+f_{2}, g_{1}+g_{2}\right) .
$$

\footnotetext{
${ }^{37}$ Dimock chama de representações em superfícies de Cauchy, mas como não são propriamente representações preferimos chamá-las por outro nome.
} 
Como é bem sabido há uma certa ambiguidade na escolha do espaço de Hilbert no qual as soluções de Klein-Gordon estão definidas quando estamos em um espaço-tempo curvo, e surge o problema que em tudo que foi feito até então tem como elemento essencial um espaço de Hilbert. Veremos no próximo paragrafo que de fato esse espaço de Hilbert é acidental, podemos abstrai-lo da construção (isso é um dos fatos que fazem dos axiomas de HaagKastler princípios naturais para quantização em espaços-tempos curvos).

A Álgebra de Weyl Sejam agora os elementos $W(f, g)$ de uma álgebra sobre $\mathfrak{F}_{0}(\mathcal{S}) \ni f, g$, que satisfazem a seguinte relação:

$$
W\left(f_{1}, g_{1}\right) W\left(f_{2}, g_{2}\right)=e^{-i\left(\left\langle f_{1}, g_{2}\right\rangle-\left\langle f_{2}, g_{1}\right\rangle\right) / 2} W\left(f_{1}+f_{2}, g_{1}+g_{2}\right),
$$

onde $\langle f, g\rangle=\int_{\mathcal{S}} f g d V_{s}$. E com esses elementos definimos ${ }^{38}$

$$
W(f)=W\left(\rho_{1} G f, \rho_{0} G f\right), \quad f \in \mathfrak{F}_{0}(\mathscr{M}) .
$$

Esses operadores satisfazem a seguinte relação

$$
\begin{gathered}
W(f) W(h)=W\left(\rho_{1} G f, \rho_{0} G f\right) W\left(\rho_{1} G h, \rho_{0} G h\right)= \\
W\left(\rho_{1} G(f+h), \rho_{0} G(f+h)\right) e^{-i\left(\left\langle\rho_{1} G f, \rho_{0} G h\right\rangle-\left\langle\rho_{1} G h, \rho_{0} G f\right\rangle\right) / 2}= \\
W(f+h) e^{-i / 2\left(-\left\langle f, G \rho_{1}^{\prime} \rho_{0} G h\right\rangle+\left\langle G \rho_{0}^{\prime} \rho_{1} G h, f\right\rangle\right)}=W(f+h) e^{-i / 2\left(\left\langle f,\left(G \rho_{0}^{\prime} \rho_{1} G-G \rho_{1}^{\prime} \rho_{0} G\right) h\right)\right.} \\
=W(f+h) e^{-i\langle f, G h\rangle / 2} .
\end{gathered}
$$

E claro que segue dessa relação que $W(0)=\mathbb{1}$, portanto, se $\langle f, G h\rangle$ for uma forma simplética não-degenerada seguirá que o conjunto $\{W(f): f \in$ $\left.\mathfrak{F}_{0}(\mathscr{M})\right\}$ é uma álgebra de Weyl, já que seria possível mostrar (como feito em seção anterior) que teríamos por necessidade $W(f)^{*}=W(-f)$.

Que $\sigma(f, g)$ é uma forma simétrica segue da linearidade de $G$ e de $\langle$,$\rangle e do fato$ do adjunto formal de $G$ ser $-G: \sigma(f, g)=\langle f, G g\rangle=-\langle G f, g\rangle=-\langle g, G f\rangle=$ $-\sigma(g, f) 39$

Por fim provaremos o seguinte teorema:

Teorema 4.1 ([13]). Sejam as $C^{*}$-álgebras $\mathscr{A}(\mathcal{O})$ geradas por

$$
\left\{W(f): f \in \mathfrak{F}_{0}(\mathscr{M}), \operatorname{supp}(f) \subset \mathcal{O}\right\},
$$

\footnotetext{
${ }^{38}$ É claro que $G f$, para todo $f \in \mathfrak{F}_{0}(\mathscr{M})$ é uma solução da equação de Klein-Gordon homogênea e então $\rho_{i} G f$ são os dados $u_{i} \in \mathfrak{F}(\mathcal{S}), i=0,1$ do problema de Cauchy.

${ }^{39}$ Para garantir a não-degeneralidade tomamos $\sigma$ sobre $\mathfrak{F}_{0}(\mathscr{M}) / \operatorname{ker}(G)$ ao invés de $\mathfrak{F}_{0}(\mathscr{M})$ somente, onde, sabemos pelas propriedades de $G$ que $\operatorname{ker} G=\left(\square+m^{2}\right) \mathfrak{F}_{0}(\mathscr{M})$. Para mais detalhes vide [5].
} 
onde $\mathcal{O}$ são conjuntos abertos e limitados de $\mathscr{M}$.

Então a $C^{*}$-álgebra $\mathscr{A}=\overline{\cup_{\mathcal{O}} \mathscr{A}(\mathcal{O})}$ satisfaz os axiomas de Haag-Kastler generalizados.

Antes de provar este teorema notamos que a rede de álgebras $\mathscr{A}(\mathcal{O})$ não depende da realização $(\mathscr{H}, W)$ sobre a superfície de Cauchy $\mathcal{S}$. Isso significa que: (1) as álgebras em diferentes superfícies de Cauchy devem ser isomórficas e ainda que (2) em uma mesma superfície de Cauchy duas álgebras geradas por diferentes realizações devem ser isomórficas 40

Um fato que se mostrará crucial tanto para essas preliminares quanto para o teorema é que a álgebra $\mathscr{A}$ pode também ser gerada por $W\left(h, h^{\prime}\right), h, h^{\prime} \in$ $\mathfrak{F}_{0}(\mathcal{S})$ : basta tomar uma solução $u$ do problema de Cauchy com os dados $h$ e $h^{\prime}$ em $\mathfrak{F}_{0}(\mathcal{S})$, e tomar uma função $f \in \mathfrak{F}_{0}(\mathscr{M})$ tal que $u=G f$, e assim $W\left(h, h^{\prime}\right)=W\left(\rho_{1} G f, \rho_{0} G f\right)=W(f)$.

Primeiro provaremos o (1): sejam duas álgebras $\mathscr{A}$ e $\tilde{\mathscr{A}}$, como as do teorema 4.1), geradas por $(\mathscr{H}, W)$ e $(\tilde{\mathscr{H}}, \tilde{W})$. Naturalmente há um *-isomorfismo entre elas, $i(\mathscr{A})=\tilde{\mathscr{A}}$; como $\mathscr{A}$ e $\tilde{\mathscr{A}}$ são geradas por $(\mathscr{H}, W)$ e $(\tilde{\mathscr{H}}, \tilde{W})$ isso significa apenas que $i\left(W\left(h, h^{\prime}\right)\right)=\tilde{W}\left(h, h^{\prime}\right)$, portanto, pela igualdade do parágrafo acima: $i(W(f))=\tilde{W}(f)$, e então, $i(\mathscr{A}(\mathcal{O}))=\tilde{\mathscr{A}}(\mathcal{O})$.

Para provar que $\mathscr{A}(\mathcal{O})$ não depende da superfície de Cauchy, usamos o seguinte resultado, cuja prova se encontrá em [13] e consiste apenas em uma conta: seja $\tilde{\rho}_{i}: \mathfrak{F}(\mathscr{M}) \rightarrow \mathfrak{F}(\tilde{\mathcal{S}}), i=0,1$ a restrição à e a derivada normal em $\tilde{\mathcal{S}}$, onde $\tilde{\mathcal{S}}$ é uma outra superfície de Cauchy, então $\tilde{\theta}=\tilde{\rho_{0}} \phi$ e $\tilde{\pi}=\tilde{\rho_{1}} \phi$ fornecem uma realização da álgebra CCR sobre a superfície de Cauchy $\tilde{\mathcal{S}}$. Como para provar a independência da álgebra gerada da superfície de Cauchy basta mostrar que duas representações em superfícies de Cauchy diferentes são iguais tomamos as representações $(\mathscr{H}, W)$ e $(\tilde{\mathscr{H}}, \tilde{W})$ onde $\tilde{W}$ é definido através $\operatorname{dos} \tilde{\theta}$ e $\tilde{\phi}$ acima. Através de algumas contas (cujo desenvolvimento explícito se encontra, novamente, em [13]) é possível mostrar que $W=\tilde{W}$.

Demonstração. Grande parte da prova da validade do axioma (1) consiste em mostrar que se $\mathcal{O}_{1}$ e $\mathcal{O}_{2}$ possuem uma separação tipo-espaço então $\sigma(f, g)=0$ para $\operatorname{supp}(f) \in \mathcal{O}_{1}$ e $\operatorname{supp}(g) \in \mathcal{O}_{2}$. Já que se isso for verdade o axioma (1) será satisfeito, pois nesse caso o que é tratado aqui seria um caso particular do que foi provado em seção anterior, viz., que álgebras de Weyl cujas formas simpléticas obedecem uma certa hipótese (a saber, que para dois índices $i$ e

\footnotetext{
${ }^{40}$ Isso parece ser trivial, já que álgebras CCR são sempre *-isomórficas. Mas devemos mostrar que esse isomorfismo é, em certo sentido, local: o isomorfismo deve levar a álgebra de uma região na outra álgebra da mesma região.
} 
$j$ temos $\left.i \perp j \Leftrightarrow \sigma\left(f_{i}, g_{j}\right)=0\right)$ são quasi-locais.

Mas isso é simples de provar: sejam duas regiões $\mathcal{O}_{1}$ e $\mathcal{O}_{2}$ com uma separação tipo-espaço e $\operatorname{sejam} \operatorname{supp}(f) \in \mathcal{O}_{1}$ e $\operatorname{supp}(g) \in \mathcal{O}_{2}$. Então, $\int_{\mathcal{S}} f G g d V_{s}=$ $\int_{\mathcal{S}} f G_{+} g d V_{s}-\int_{\mathcal{S}} f G_{-} g d V_{s}$, porém $\operatorname{supp}\left(G_{ \pm} g\right) \subset J^{ \pm}(\operatorname{supp} g)$, e como não há curva causal que una $\mathcal{O}_{1} \ni \operatorname{supp} f$ e $\mathcal{O}_{2} \ni \operatorname{supp} g, \operatorname{supp} f \cap J^{ \pm}(\operatorname{supp} g)=\emptyset$, i.e., $\operatorname{supp}(f) \cap \operatorname{supp}\left(G_{ \pm} g\right)=\emptyset$ de modo que $\langle f, G g\rangle=0=\sigma(f, g)$. Isso prova o axioma (1).

Para mostrar a validade do axioma (2) basta tomarmos uma realização $(\mathscr{H}, W)$ sobre $\mathcal{S} \subset \mathscr{M}$, que gera a rede de álgebras $\mathscr{A}(\mathcal{O})$ e outra realização $\left(\mathscr{H}^{\prime}, W^{\prime}\right)$ sobre $\mathcal{S}^{\prime} \subset \mathscr{M}^{\prime}$, que gera a rede de álgebras $\mathscr{A}^{\prime}\left(\mathcal{O}^{\prime}\right)$. Se mostrarmos que $\mathscr{A}(\mathcal{O})=\mathscr{A}^{\prime}(k(\mathcal{O}))$ então a conformidade com o axioma (2) fica estabelecida, já que quaisquer outras álgebras serão isomorfas a estas.

Tomamos as seguintes realizações: realização de $\mathscr{A}:(\mathscr{H}, W(f, g), f, g \in$ $\left.\mathfrak{F}_{0}(\mathcal{S})\right)$. Realização de $\mathscr{A}^{\prime}:\left(\mathscr{H}, W^{\prime}(f, g), f, g \in \mathfrak{F}_{0}(k(\mathcal{S}))\right)$, onde $W^{\prime}$ é dado por, para $f, g \in \mathfrak{F}_{0}(k(\mathcal{S}))$ :

$$
W^{\prime}(f, g)=W\left(k_{0}^{*} f, k_{0}^{*} g\right)=W\left(f \circ k_{0}, g \circ k_{0}\right),
$$

onde $k_{0}: \mathcal{S} \rightarrow k(\mathcal{S})$ é o difeomorfismo induzido quando restringimos a isometria aos domínios e imagens adequados. Agora vejamos como podemos escrever $\tilde{G}: \mathfrak{F}_{0}\left(\mathscr{M}^{\prime}\right) \rightarrow \mathfrak{F}\left(\mathscr{M}^{\prime}\right)$, a função de Green no espaço-tempo $\left(\mathscr{M}^{\prime}, g^{\prime}\right)$ em termos da função de Green $G$, e também os operadores derivada e restrição $\tilde{\rho}_{i}: \mathfrak{F}\left(\mathscr{M}^{\prime}\right) \rightarrow \mathfrak{F}\left(\mathcal{S}^{\prime}\right)$ em termos de $\rho_{i}$. Para isso usaremos os difeomorfismos $k: \mathscr{M} \rightarrow \mathscr{M}^{\prime}$ e $k_{0}: \mathcal{S} \rightarrow \mathcal{S}^{\prime}$ para transformar funções de um espaço em funções do outro espaço.

Seja $f \in \mathfrak{F}_{0}\left(\mathscr{M}^{\prime}\right)$, então $f \circ k \in \mathfrak{F}_{0}(\mathscr{M})$, e $G(f \circ k) \in \mathfrak{F}(\mathscr{M})$. Utilizando de novo o difeomorfismo $k$ para trazer $G(f \circ k)$ para o espaço de funções em $\mathscr{M}^{\prime}$ escrevemos $\tilde{G} f=G(f \circ k) \circ k^{-1}$. Da mesma forma para $\rho_{i}$, seja $f \in \mathfrak{F}(\mathscr{M})$ então $\rho_{i}(f \circ k) \in \mathfrak{F}(\mathcal{S})$, então $\tilde{\rho}_{i}(f)=\rho_{i}(f \circ k) \circ k_{0}^{-1}$.

Então, para $f \in \mathfrak{F}_{0}\left(\mathscr{M}^{\prime}\right)$

$$
\begin{gathered}
W^{\prime}(f)=W^{\prime}\left(\tilde{\rho}_{1} \tilde{G} f, \tilde{\rho}_{0} \tilde{G} f\right) \\
=W^{\prime}\left(\rho_{1}\left(G(f \circ k) \circ k^{-1} \circ k\right) \circ k_{0}^{-1}, \rho_{0}\left(G(f \circ k) \circ k^{-1} \circ k\right) \circ k_{0}^{-1}\right) \\
=W\left(\rho_{1} G(f \circ k), \rho_{0} G(f \circ k)\right)=W(f \circ k) .
\end{gathered}
$$

Isso mostra que $\mathfrak{A}\left(k^{-1}(\mathcal{O})\right)=\mathfrak{A}^{\prime}(\mathcal{O})$, como desejado.

O axioma (3) segue da simplicidade das álgebras CCR: pela simplicidade as álgebras CCR não possuem ideais não-triviais, ou seja, se $W(f) \in \mathcal{A} \subset \mathscr{A}$ então,

$$
W(f) W(g) \subset \mathcal{A}, \forall W(g) \in \mathscr{A} \Rightarrow \mathcal{A}=\mathscr{A} \text { ou } \mathcal{A}=\{0\} .
$$


De fato, se $W(f) \in \mathcal{A} \subset \mathscr{A}$ então $f \in H_{1} \subset \mathfrak{F}_{0}(\mathscr{M})$. Mas não existe um subespaço de $\mathfrak{F}_{0}(\mathscr{M})$ tal que para todo $g \in \mathfrak{F}_{0}(\mathscr{M}), f+g \in H_{1}$.

Assim, suponha que $\mathscr{A}$ seja redutível, então existe um $\mathcal{A} \subset \mathscr{A}$ tal que para todo $W(f) \in \mathcal{A}$ vale que

$$
\pi(W(f)) \pi(W(g)) \in \pi(\mathcal{A}), \quad \forall W(g) \in \mathscr{A},
$$

onde $\pi$ é uma representação de $\mathscr{A}$.

Mas como $\pi$ é um *-morfismo, $\pi(W(f)) \pi(W(g))=\pi(W(f) W(g))$. No entanto se $W(f) W(g) \in \mathcal{A}$ para todo $W(g)$ em $\mathscr{A}$ então $\mathcal{A}=\mathscr{A}$. Ou seja, $\pi$ deve necessariamente ser irredutível.

Que há uma representação fiel de $\mathscr{A}$ : como as álgebras CCR são únicas a não ser por um *-isomorfismo dada uma representação $\pi$ de $\mathscr{A}$ existe um único ${ }^{*}$-isomorfismo $\alpha$ entre $\mathscr{A}$ e $\pi(\mathscr{A})$ tal que $\alpha(W(f))=\pi(W(f))$, para todo $f$. Então $\alpha=\pi$. Então, o núcleo de $\pi$ é zero.

Por fim, o axioma (4) se prova assim: seja $\operatorname{supp} f \subset \mathcal{O}_{1}$, como, $\operatorname{supp}(G f) \subset$ $\bigcup J^{ \pm}(\operatorname{supp} f)$, os operadores $\rho_{i}$ restringem essa união à superfície de Cauchy: $\operatorname{supp}\left(\rho_{i} G f\right) \subset \bigcup J^{ \pm}(\operatorname{supp} f) \cap \mathcal{S}$ e pela hipótese de que $\mathcal{O}_{1}$ depende causalmente de $\mathcal{O}_{2}: \operatorname{supp}\left(\rho_{i} G f\right) \subset \mathcal{O}_{2}$. Tomamos um $f_{2} \in \mathfrak{F}_{0}\left(\mathcal{O}_{2}\right)$ tal que $G f=G f_{2}{ }^{41}$ Assim, $\operatorname{supp}\left(\rho_{i} G f_{2}\right) \subset \mathcal{O}_{2}$ e então $W(f)=W\left(f_{2}\right)$. Portanto, $W(f) \subset \mathscr{A}\left(\mathcal{O}_{2}\right)$. Ou seja, $\mathscr{A}\left(\mathcal{O}_{1}\right) \subset \mathscr{A}\left(\mathcal{O}_{2}\right)$.

Exemplo Neste exemplo construiremos forma simplética $\sigma$, como feito acima: $\sigma(f, g)=\langle f, G g\rangle$, para campos bosônicos em espaços de Minkowski $\left(\mathbb{R}^{4}, \eta\right)$, ou seja, usaremos o $G$ calculado no exemplo da seção (3.2.4) e veremos elementos das álgebras CCR-Weyl que quantizam esses campos.

$$
\begin{gathered}
\sigma(f, g)=\langle f, G g\rangle=\int_{\mathbb{R}^{4}} f(x)(G g)(x) d^{4} x= \\
=\left.\frac{i}{2 \pi} \int_{\mathbb{R}^{4}} \int_{\mathbb{R}^{3}} \frac{1}{2 \omega_{p}} f(x)\left(e^{i p x} \hat{g}(p)-e^{-i p x} \hat{g}(-p)\right)\right|_{p_{0}=\omega_{p}} d^{3} p d^{4} x .
\end{gathered}
$$

Mas $\hat{f}(p)=\left(1 /(2 \pi)^{2}\right) \int e^{-i p x} f(x) d^{4} x$, então,

$$
\sigma(f, g)=\left.2 \pi i \int_{\mathbb{R}^{3}} \frac{1}{2 \omega_{p}}(\hat{f}(-p) \hat{g}(p)-\hat{f}(p) \hat{g}(-p))\right|_{p_{0}=\omega_{p}} d^{3} p .
$$

É claro que $\sigma(f, g) \in \mathbb{R}$, pois $\overline{\hat{f}(p)}=\hat{f}(-p)$. Os observáveis são os operadores auto-adjuntos.

\footnotetext{
${ }^{41} \mathrm{~A}$ prova que se pode sempre fazer isso é o Lema A.3 de [13]
} 
E ainda, $\left.\int\left(1 / 2 \omega_{p}\right) \overline{\hat{f}}(p) \hat{g}(p)\right|_{p_{0}=\omega_{p}} d^{3} p$ é o produto interno relativisticamente invariante do espaço de Hilbert $L^{2}\left(\mathbb{R}^{3}, d^{3} p / 2 \omega_{p}\right)$, chamemos esse produto de $\langle f, g\rangle$. Portanto

$$
\sigma(f, g)=2 \pi i(\langle f, g\rangle-\langle g, f\rangle)=-4 \pi \Im\langle f, g\rangle .
$$

Então,

$$
\begin{gathered}
W(f) W(g)=e^{-i \sigma(f, g) / 2} W(f+g)= \\
=e^{2 \pi i \Im\langle f, g\rangle} W(f+g) .
\end{gathered}
$$


5 Estados Coerentes em Álgebras CCR 
Nesta seção veremos como é possível definir (segundo [37]) estados coerentes em uma álgebra CCR qualquer.

Seja a álgebra quasi-local $\mathscr{A}=\bigcup_{\mathcal{O}} \mathscr{A}(\mathcal{O}), \mathscr{A}(\mathcal{O})$ gerada por $\{W(f): \operatorname{supp} f \subset$ $\mathcal{O}\}$, e os $W(f)$ são tais que $W(f)^{*}=W(-f), W(f) W(g)=e^{-i \sigma(f, g) / 2} W(f+$ $g$ ), como foi definida na seção (2.2.2).

Seja $(H, \sigma)$ o espaço simplético de relevância para a álgebra quasi-local $\mathscr{A}$. Seja $J$ uma estrutura complexa em $(H, \sigma)$, ou seja, $J: H \rightarrow H$, linear, $J^{2}=-\mathbb{1}, \sigma(J f, f)<0$ e $\sigma(J f, J g)=\sigma(f, g)$. O espaço simplético com essa estrutura é a complexificação de $H$, e chamamos esse espaço de $\mathscr{H}$. Definindo a aplicação (.,.) : $\mathscr{H} \times \mathscr{H} \rightarrow \mathbb{C}$ por

$$
(f, g)=\sigma(f, J g)+i \sigma(f, g) .
$$

Obtemos um espaço pre-Hilbert $\mathscr{H}{ }^{42}$ E claro, temos a relação $\sigma(f, g)=$ $\Im(f, g)$. É claro que $(.,$.$) é um produto interno, já que$

$$
\begin{array}{r}
(f, g)=\sigma(f, J g)+i \sigma(f, g)=-\sigma(J g, f)-i \sigma(g, f)= \\
=-\sigma(J J g, J f)-i \sigma(g, f)=\overline{(g, f)}
\end{array}
$$

E como $\sigma$ é bilinear inferimos facilmente que $\left(f, g_{1}+g_{2}\right)=\left(f, g_{1}\right)+\left(f, g_{2}\right)$ e analogamente para a primeira componente. Vejamos a multiplicação por um complexo $z=a+i b$ :

$$
\begin{aligned}
& (f, z g)=\sigma(f, J \overbrace{(a+i b) g}^{=a g+b J g}+i \sigma(f,(a+i b) g)=a \sigma(f, J g)+b \sigma\left(f, J^{2} g\right) \\
& +i[a \sigma(f, g)+b \sigma(f, J g)] \text {. } \\
& =\sigma(f, J g)(a+i b)+i \sigma(f, g)(a+i b)=z(f, g)
\end{aligned}
$$

E na primeira componente:

$$
\begin{gathered}
(z f, g)=\sigma((a+i b) f, J g)+i \sigma((a+i b) f, g)=a \sigma(f, J g) \\
+b \sigma(J f, J g)+i[a \sigma(f, g)+b \overbrace{\sigma(J f, g)}^{=-\sigma(f, J g)}] \\
=\sigma(f, J g)(a-i b)+\sigma(f, g)(b+i a)=\sigma(f, J g)(a-i b)+i \sigma(f, g)(a-i b)=\bar{z}(f, g) . \\
\text { E claro: }(f, f)=\sigma(f, J f)+i \sigma(f, f)=\sigma(f, J f) \geq 0 .
\end{gathered}
$$

\footnotetext{
${ }^{42}$ Poderíamos, se mais claridade fosse necessária, escrever o produto interno e a norma com um subscrito, indicando qual espaço simplético estamos transformando em espaço de Hilbert.
} 
Exemplo Retomando o exemplo anterior, vejamos que podemos de fato recuperar o produto interno $\left(\mathrm{em} L^{2}\left(\mathbb{R}^{4}, d^{3} p / \omega_{p}\right)\right.$ ) tomando um $J$ apropriado:

$$
\sigma(f, g)=\left.\pi i \int_{\mathbb{R}^{3}} \frac{1}{\omega_{p}}(\hat{f}(-p) \hat{g}(p)-\hat{f}(p) \hat{g}(-p))\right|_{p_{0}=\omega_{p}} d^{3} p .
$$

Se calcularmos $\sigma(f, J f)$ deveremos obter $\|f\|^{2}$. Chamemos $\hat{f}_{J}(p)$ a transformada de Fourier de $J f: \hat{f}_{J}=\widehat{J f}$. Então,

$$
\sigma(f, J f)=\left.\pi i \int \frac{1}{\omega_{p}}\left(\hat{f}(-p) \hat{f}_{J}(p)-\hat{f}(p) \hat{f}_{J}(-p)\right)\right|_{p_{0}=\omega_{p}} d^{3} p
$$

Se tomarmos $\hat{f}_{J}(p)=-i \hat{f}(p)$ - e portanto $\hat{f}(-p)=\overline{\hat{f}_{J}(p)}=i \overline{\hat{f}}(p)$ :

$$
\sigma(f, J f)=\left.\pi \int \frac{1}{\omega_{p}} \overline{\hat{f}}(p) \hat{f}(p)\right|_{p_{0}=\omega_{p}} d^{3} p=\pi\|f\|^{2} .
$$

O vácuo Um estado que é de particular importância para a física quântica é o estado que corresponde ao vácuo que em um espaço de Fock é definido como o estado sem nenhuma partícula: $\psi_{0}=(1,0,0, \ldots)$. O valor esperado de $W(f)=\exp (i \Phi(f))$ em $\psi_{0}$ é $\exp \left(-\|f\|^{2} / 4\right)$ :

$$
\left(\psi_{0}, W(f) \psi_{0}\right)=\left(\psi_{0}, e^{i\left(a(f)+a^{*}(f)\right) / \sqrt{2}} \psi_{0}\right)=\left(\psi_{0}, e^{-\|f\|^{2} / 4} e^{i a(f) / \sqrt{2}} e^{i a^{*}(f) / \sqrt{2}} \psi_{0}\right),
$$

onde usamos a fórmula de Baker-Campbell-Hausdorff para dois operadores cujo comutador é um múltiplo de identidade, ou seja, para $[x, y]=c \mathbb{1}$ nos diz que $e^{x+y}=e^{-\frac{1}{2}[x, y]} e^{x} e^{y}=e^{\frac{1}{2}[x, y]} e^{y} e^{x}$. E como como vimos na seção 2.1.1, no espaço de Fock $a(f) \psi_{0}=0$, então

$$
\exp (a(f)) \psi_{0}=(\mathbb{1}+a(f)+a(f) a(f) / 2+\cdots) \psi_{0}=\psi_{0} .
$$

Então $\left(\psi_{0}, W(f) \psi_{0}\right)=e^{-\|f\|^{2} / 4}$. É natural, portanto, definir o estado vetorial $\psi_{0}$ de forma algébrica como sendo o funcional linear $\psi_{0}: \mathscr{A} \rightarrow \mathbb{C}$ tal que $\psi_{0}(W(f))=\exp \left(-\|f\|^{2} / 4\right)$. E, de fato, dado este funcional linear podemos sempre achar um estado vetorial tal que $\psi_{0}(W(f))=\left(\psi_{0}, W(f) \psi_{0}\right)$ graças ao teorema de representação GNS43 e este estado vetorial será o vácuo tradicional que por sua vez definirá os operadores de criação e aniquilação: teremos, assim, ao menos em principio, uma correspondência entre a álgebra CCR abstrata (talvez com algum conteúdo relativístico) e partículas quânticas. No entanto, o que ocorre no caso de álgebras CCR gerais é que até mesmo

\footnotetext{
${ }^{43}$ Vide, por exemplo, [5], Teorema 8.1.3
} 
antes de representarmos os estados algébricos em espaços de Hilbert através da representação GNS é que devemos especificar uma estrutura complexa $J$ que tornará o espaço simplético sobre o qual $\mathscr{A}$ está definida em um espaço de Hilbert. Ou seja, dada a álgebra CCR $\mathscr{A}$ definida sobre $(H, \sigma)$ e dada a estrutura complexa $J: H \rightarrow H$ podemos tornar $H$ em um espaço de Hilbert complexo $\mathscr{H}$, e este produto interno é central na definição do vácuo, que em geral é o estado $\psi_{0}: \mathscr{A} \rightarrow \mathscr{A}$

$$
\psi_{0}(W(f))=\exp \left(-(f, f)_{J} / 4\right)
$$

E é um fato que para espaços simpléticos $(H, \sigma)$ de dimensão infinita há infinitas estruturas complexas diferentes, e cada uma fornecerá um espaço de Hilbert diferente. Assim, há uma ambiguidade em como a álgebra CCR se realiza em um espaço de Hilbert que garante uma interpretação de partículas. Há muitas formas de se interpretar esse fenomeno que aparece na formulação algébrica da teoria quântica de campos (por exemplo, [12] argumenta que essas representações não equivalentes fornecem perspectivas complementares do sistema físico).

Uma exigência importante para qualquer limite clássico é que nessa transição essa ambiguidade seja perdida e uma única concepção de partícula deve existir para qualquer espaço de Hilbert que seja escolhido. Veremos que esse é de fato o que ocorre no limite semi-clássico por estados coerentes.

O Estado Coerente Seja um funcional linear $l: \mathscr{H} \rightarrow \mathbb{R}$. Definimos um estado coerente em $\mathscr{A}$ como o funcional linear (que depende da estrutura complexa $J$, mas não a especificamos) $\psi_{l}: \mathscr{A} \rightarrow \mathbb{C}$ dado por ${ }^{44}$

$$
\psi_{l}(W(f))=e^{i l(f)} e^{-(f, f) / 4}=e^{i l(f)} e^{-\|f\|^{2} / 4} .
$$

É importante notar que o estado acima está definido de uma forma completamente algébrica, apesar de sua inspiração — e até seu significado- fazer referencia a estados vetoriais. E a determinação da forma de $l$, que será feita a seguir fazendo uma equivalência entre esse estado e estados vetoriais ,pode ser vista, em verdade, como um procedimento inteiramente prático, do qual podemos abstrair a forma de $l$ e já tomá-la como definição.

A interpretação de $\psi_{l}$ é imediata se considerarmos a forma de $l$ abaixo, (17): o termo $\exp \left(-\|f\|^{2} / 4\right)$ representa o vácuo e o termo $\exp (l(f))$ representa a

\footnotetext{
${ }^{44} \mathrm{O}$ que segue é de fato um estado: $\psi_{l}$ é linear por definição, e $\psi_{l}\left(W(f) W(f)^{*}\right)=$ $\psi_{l}(W(0))=1=\psi_{l}(\mathbb{1})$.
} 
ação do operador deslocamento 45

Para determinar a forma de $l(f)$ vamos comparar o estado algébrico definido acima com um estado coerente em um espaço de Hilbert advindo da realização da álgebra $\mathscr{A}$ em uma superfície de Cauchy (vide seção (4.2)). Neste caso o operador que geram os estados coerentes é precisamente $W(\alpha, \beta)$, ou seja, $W(\alpha, \beta)|0\rangle=|\alpha, \beta\rangle$.

Assim, espera-se que a relação $\psi_{l}(W(f))=\langle\alpha, \beta|W(f)| \alpha, \beta\rangle$ seja válida 46 . Como $W(f)=W\left(f_{1}, f_{0}\right)$, onde $f_{i}=\rho_{i} G f$, então,

$$
W(\alpha, \beta)^{*} W\left(f_{1}, f_{0}\right) W(\alpha, \beta)=e^{-i\left(\left\langle f_{1}, \beta\right\rangle-\left\langle\alpha, f_{0}\right\rangle\right)} W\left(f_{1}, f_{0}\right) .
$$

Por outro lado $W(f)=e^{i \Phi(f)}$, onde $\Phi(f)=\theta\left(f_{1}\right)-\pi\left(f_{0}\right)$, e como visto em (4.2) $[\Phi(f), \Phi(g)]=-i\langle f, G g\rangle=-i \sigma(f, g)$. Portanto,

$$
\begin{gathered}
{[\Phi(f), \Phi(f)]=-i \Im(f, f)=0} \\
{[\Phi(f), i \Phi(J f)]=\sigma(f, J f)=\Re(f, f)=(f, f)=\|f\|^{2} .}
\end{gathered}
$$

Assim, neste espaço de Hilbert - agora complexo — tomamos os operadores de aniquilação e criação definidos através de $a_{\mathbb{C}}(f)=2^{-1 / 2}\left(\Phi(f)-i \Pi(f) 4^{47}\right.$, onde $\Pi(f)=\Phi(J f)$, que satisfazem a relação $\left[a_{\mathbb{C}}(f), a_{\mathbb{C}}^{*}(f)\right]=\|f\|^{2}$. Escrevendo $\Phi$ em termos de $a_{\mathbb{C}}(f), \Phi(f)=2^{-1 / 2}\left(a_{\mathbb{C}}(f)+a_{\mathbb{C}}(f)^{*}\right)$ e utilizando a fórmula de Baker-Campbell-Hausdorff podemos escrever $W(f)$ como

$$
W(f)=e^{-\|f\|^{2} / 4} e^{i a_{\mathbb{C}}^{*}(f) / \sqrt{2}} e^{i a_{\mathbb{C}}(f) / \sqrt{2}} .
$$

Mas o vácuo é precisamente o estado que $a_{\mathbb{C}}(f)|0\rangle=0$, então, $e^{i a_{\mathbb{C}}(f) / \sqrt{2}}|0\rangle=$ $\left(\mathbb{1}+i a_{\mathbb{C}}(f) / \sqrt{2}+\left(i a_{\mathbb{C}}(f) / \sqrt{2}\right)^{2}+\cdots\right)|0\rangle=|0\rangle$.

Portanto,

$$
\langle\alpha, \beta|W(f)| \alpha, \beta\rangle=e^{i\left(\left\langle f_{1}, \beta\right\rangle-\left\langle\alpha, f_{0}\right\rangle\right)} e^{-\|f\|^{2} / 4} .
$$

É claro que

$$
l(f)=\left\langle f_{1}, \beta\right\rangle-\left\langle\alpha, f_{0}\right\rangle=\left\langle\rho_{1} G f, \beta\right\rangle-\left\langle\alpha, \rho_{0} G f\right\rangle,
$$

\footnotetext{
${ }^{45}$ No contexto da mecânica quântica sua forma é bem conhecida: $D(\alpha)=\exp \left(\alpha \hat{a}^{*}-\right.$ $\bar{\alpha} \hat{a})$, cuja álgebra é basicamente a álgebra CCR-Weyl - e isso nos permite definir estados coerentes usando elementos da álgebra de uma forma natural, como faremos abaixo.

${ }^{46}$ Podemos sempre fazer isso, graças ao teorema de representação GNS: dado um estado em uma álgebra há uma representação desta álgebra num espaço de Hilbert cujo estado algébrico corresponde ao estado vetorial, de forma única (à não ser por uma equivalência unitária).

${ }^{47}$ Escrevemos $a$ com um índice $\mathbb{C}$ para diferencia-lo de outro operador $a$ definido através da realização na superfície de Cauchy $(\mathscr{H}, \theta, \pi)$.
} 
Onde $\alpha, \beta \in \mathfrak{F}_{0}(\mathcal{S})$ e $f \in \mathfrak{F}_{0}(\mathscr{M})$. Se tomarmos $\alpha=u_{1}$ e $\beta=u_{0}$, onde $u_{i}$ são os dados de um problema de Cauchy: ou seja, se $u$ é solução de $P u=0$ e $\rho_{i} u=u_{i}$, então, (17) é simplesmente - como visto na seção (3.2.4), equação (12) -

$$
l(f)=\langle u, f\rangle .
$$

\subsection{Relações de Incerteza}

Sendo assim, provemos as relações de mínima incerteza que um estado coerente deve satisfazer utilizando o estado $|\alpha, \beta\rangle=W(\alpha, \beta) \psi_{0}$.

Seja $A$ um observável, define-se $\sigma_{A}$, a variância de $A$ no estado $\alpha$, por

$$
\sigma_{A}=\sqrt{\left\langle A^{2}\right\rangle-\langle A\rangle^{2}}
$$

onde $\langle A\rangle=\langle\alpha, \beta|A| \alpha, \beta\rangle$ Dado dois observáveis $A$ e $B$, a relação de incerteza nos diz que

$$
\sigma_{A}^{2} \sigma_{B}^{2} \geq\left(\frac{1}{2 i}\langle[A, B]\rangle\right)^{2}
$$

Essa desigualdade deve ser uma igualdade quando calculamos em $A=\Phi(f)$ e $B=\Phi(i f)$, ou seja, os geradores da álgebra de Weyl que fazem o papel de posição e momento 48

Antes de mostrar que de fato $\Phi(f)$ e $\Pi(f)$ são estados de mínima incerteza desenvolveremos algumas propriedades de $W(f, g)=\exp (i(\theta(f)-\pi(f)))$ que serão importantes no que segue para além desta seção.

Sejam $f=\pi$ e $g=\phi$ o momento e solução da equação de Klein-Gordon (i.e., $\left.\pi=u_{1}, \phi=u_{0}\right)$, e seja $\alpha=(\phi-i \pi) / \sqrt{2}$, procuremos escrever $W(\pi, \phi)=$ $\exp i(\theta(\pi)-\pi(\phi))$ em termos de $a=(\theta+i \pi) / \sqrt{2}$ e $\alpha$ :

$$
\begin{gathered}
\theta(\pi)-\pi(\phi)=\theta\left(\frac{1}{\sqrt{2} i}(\alpha-\bar{\alpha})\right)-\pi\left(\frac{1}{\sqrt{2}}(\alpha+\bar{\alpha})\right)= \\
=\frac{1}{\sqrt{2}}\left(-i \theta(\alpha)+i \theta(\bar{\alpha})+i^{2} \pi(\alpha)+i^{2} \pi(\bar{\alpha})\right)= \\
=\frac{i}{\sqrt{2}}(-(\theta(\alpha)-i \pi(\alpha))+\theta(\bar{\alpha})+i \pi(\bar{\alpha}))=i\left(a(\bar{\alpha})-a^{*}(\alpha)\right) \\
\Rightarrow W(\alpha)=\exp \left(a^{*}(\alpha)-a(\bar{\alpha})\right) .
\end{gathered}
$$

Ou seja, precisamente o que esperaríamos para $W$ que define estados coerentes. Antes de prosseguir para a determinação de igualdades que serão

\footnotetext{
${ }^{48}$ Note que $\Phi(f)$ e $\Phi(i f)$ geram álgebras que não comutam, já que $\Im(f, i f)=\Im\left(i\|f\|^{2}\right)=$ $\|f\|^{2}$, que não é em geral zero.
} 
fundamentais para a analogia que será feita entre o teorema de Hepp e o limite semi-clássico para estados algébricos bem como para a prova de que posição e momento são estados de incerteza minima, vejamos quais as relações de comutação de $a$ :

$$
\begin{gathered}
{[a(f), a(g)]=\frac{1}{2}[\theta(f)+i \pi(f), \theta(g)+i \pi(g)]=\frac{i}{2}([\theta(f), \pi(g)]-[\theta(g), \pi(f)])} \\
=\frac{i}{2}(i\langle f, g\rangle-i\langle f, g\rangle)=0 .
\end{gathered}
$$

Analogamente para $\left[a^{*}(f), a^{*}(g)\right]$. Agora, $\left[a(f), a^{*}(g)\right]$ :

$$
\begin{aligned}
{\left[a(f), a^{*}(g)\right] } & =\frac{1}{2}[\theta(f)+i \pi(f), \theta(g)-i \pi(g)]=\frac{-i}{2}([\theta(f), \pi(g)]+[\theta(g), \pi(f)]) \\
& =\frac{-i}{2}(i\langle f, g\rangle+i\langle f, g\rangle) \Rightarrow\left[a(f), a^{*}(g)\right]=\langle f, g\rangle .
\end{aligned}
$$

Para saber o valor de $\psi_{l}(\Phi(f))$ precisamos saber $W(\alpha)^{*} \Phi(f) W(\alpha)$, ou seja, precisamos saber o valor de expressões da forma $a(f) W(\alpha)$ e $a^{*}(f) W(\alpha)$. Pela fórmula de Baker-Campbell-Hausdorff:

$$
W(\alpha)=e^{a^{*}(\alpha)+(-a(\bar{\alpha}))}=e^{-\langle\alpha, \bar{\alpha}\rangle / 2} e^{a^{*}(\alpha)} e^{-a(\bar{\alpha})} .
$$

Então, essencialmente queremos saber como passar $a$ ou $a^{*}$ para a direta de $\exp \left(a^{*}(\alpha)\right)$ e $\exp (-a(\bar{\alpha}))$, respectivamente.

$$
\begin{gathered}
{\left[a(f), a^{*}(\alpha)^{n}\right]=n\langle f, \alpha\rangle a^{*}(\alpha)^{n-1}=a(f) a^{*}(\alpha)^{n}-a^{*}(\alpha)^{n} a(f)} \\
\Rightarrow a(f) a^{*}(\alpha)^{n}=n\langle f, \alpha\rangle a^{*}(\alpha)^{n-1}+a^{*}(\alpha)^{n} a(f) .
\end{gathered}
$$

Do mesmo modo

$$
\begin{array}{r}
{\left[a^{*}(f), a(\bar{\alpha})^{n}\right]=-n\langle f, \bar{\alpha}\rangle a(\bar{\alpha})^{n-1} \Rightarrow a^{*}(f) a(\bar{\alpha})^{n}=} \\
=-n\langle f, \alpha\rangle a(\bar{\alpha})^{n-1}+a(\bar{\alpha})^{n} a^{*}(f) .
\end{array}
$$

Assim,

$$
\begin{gathered}
a(f) e^{a^{*}(\alpha)}=\sum \frac{1}{n !} a(f) a^{*}(\alpha)^{n}=\langle f, \alpha\rangle \sum \frac{n}{n(n-1) !} a^{*}(\alpha)^{n-1}+e^{a^{*}(\alpha)} a(f) \\
\therefore a(f) e^{a^{*}(\alpha)}=e^{a^{*}(\alpha)}(\langle f, \alpha\rangle+a(f)) .
\end{gathered}
$$

E analogamente

$$
a^{*}(f) e^{-a(\bar{\alpha})}=e^{-a(\bar{\alpha})}\left(\langle f, \bar{\alpha}\rangle+a^{*}(f)\right) .
$$


Isso essencialmente nos diz que

$$
W(\alpha)^{*} a(f) W(\alpha)=\langle f, \alpha\rangle+a(f), \quad W(\alpha)^{*} a^{*}(f) W(\alpha)=\langle f, \bar{\alpha}\rangle+a^{*}(f) .
$$

Finalmente, calculemos agora $W(\alpha)^{*} \Phi(f) W(\alpha)$ :

$$
\begin{array}{r}
\Phi(f)=\theta\left(f_{1}\right)-\pi\left(f_{0}\right)=\frac{1}{\sqrt{2}}\left(a\left(f_{1}\right)+a^{*}\left(f_{1}\right)+i a\left(f_{0}\right)-i a^{*}\left(f_{0}\right)\right) \\
\Rightarrow W(\alpha)^{*} \Phi(f) W(\alpha)=\frac{W(\alpha)^{*}}{\sqrt{2}}\left(a\left(f_{1}\right) W(\alpha)+a^{*}\left(f_{1}\right) W(\alpha)+\right. \\
\left.i a\left(f_{0}\right) W(\alpha)-i a^{*}\left(f_{0}\right) W(\alpha)\right) \\
=: F_{\alpha}(f) \\
=\underbrace{\theta\left(f_{1}\right)-\pi\left(f_{0}\right)}_{=\Phi(f)}+\overbrace{\frac{1}{\sqrt{2}}\left(\left\langle f_{1}, \alpha\right\rangle+\left\langle f_{1}, \bar{\alpha}\right\rangle+i\left\langle f_{0}, \alpha\right\rangle-i\left\langle f_{0}, \bar{\alpha}\right\rangle\right)} .
\end{array}
$$

Portanto,

$$
W(\alpha)^{*} \Phi(f) W(\alpha)=\Phi(f)+F_{\alpha}(f)
$$

Para que não hajam mais distrações nesta seção veremos o significado de $F_{\alpha}$ na próxima seção somente. Prossigamos, assim, para o cálculo explicito de $\sigma_{\Phi(f)}, \sigma_{\Pi(f)}$, etc.:

$$
\begin{gathered}
\langle\alpha|\Phi(f)| \alpha\rangle=\langle 0|\Phi(f)| 0\rangle+F_{\alpha}(f) \\
\Rightarrow\langle\alpha|\Phi(f)| \alpha\rangle^{2}=\langle 0|\Phi(f)| 0\rangle^{2}+2 F_{\alpha}(f)\langle 0|\Phi(f)| 0\rangle+F_{\alpha}^{2}(f) .
\end{gathered}
$$

Por outro lado, $W(\alpha) \Phi(f)^{2} W(\alpha)=W(\alpha)^{*} \Phi(f) W(\alpha) W(\alpha)^{*} \Phi(f) W(\alpha)=$ $\left(\Phi(f)+F_{\alpha}(f)\right)^{2}$, portanto,

$$
\left\langle\alpha\left|\Phi(f)^{2}\right| \alpha\right\rangle=\left\langle 0\left|\Phi(f)^{2}\right| 0\right\rangle+2 F_{\alpha}(f)\langle 0|\Phi(f)| 0\rangle+F_{\alpha}^{2}(f) .
$$

Portanto,

$$
\sigma_{\Phi(f)}^{2}=\left\langle 0\left|\Phi(f)^{2}\right| 0\right\rangle-\langle 0|\Phi(f)| 0\rangle^{2} .
$$

É claro que, como $\Phi(f)=\left(a_{\mathbb{C}}(f)+a_{\mathbb{C}}^{*}(f)\right) / \sqrt{2},\langle\Phi(f)\rangle_{0}=0$. Resta saber o primeiro termo:

$$
\begin{aligned}
\Phi(f)^{2} & =\frac{1}{2}\left(a_{\mathbb{C}}(f)^{2}+a_{\mathbb{C}}(f) a_{\mathbb{C}}^{*}(f)+a_{\mathbb{C}}^{*}(f) a_{\mathbb{C}}(f)+a_{\mathbb{C}}^{*}(f)^{2}\right) \\
& =\frac{1}{2}\left(a_{\mathbb{C}}(f)^{2}+2 a_{\mathbb{C}}^{*}(f) a_{\mathbb{C}}(f)+a_{\mathbb{C}}^{*}(f)+\|f\|^{2}\right),
\end{aligned}
$$


onde a norma de $f$ advém do comutador $\left[a_{\mathbb{C}}(f), a_{\mathbb{C}}^{*}(f)\right]=\|f\|^{2}$. Então,

$$
\left\langle\Phi(f)^{2}\right\rangle_{0}=\frac{\|f\|^{2}}{2}=\sigma_{\Phi(f)}^{2} .
$$

Como $\|f\|^{2}=(f, f)=\sigma(f, J f)$ e $\sigma(J f, J g)=\sigma(f, g), \forall f, g$, obtemos o mesmo resultado para $\sigma_{\Pi(f)}^{2}$. Calculemos agora $(\langle[\Phi(f), \Pi(f)]\rangle / 2 i)^{2}$ :

$$
[\Phi(f), \Pi(f)]=[\Phi(f), \Phi(J f)]=-i \sigma(f, J f)=-i\|f\|^{2} .
$$

Portanto,

$$
\left(\frac{\langle[\Phi(f), \Pi(f)]\rangle}{2 i}\right)^{2}=\frac{\|f\|^{4}}{4}=\sigma_{\Phi(f)}^{2} \sigma_{\Pi(f)}^{2} .
$$

Como queríamos demonstrar. Notemos ainda que no fim nada depende de $\alpha$, ou seja, não depende da solução em torno da qual o estado coerente está definido, mas apenas de uma função que define o próprio momento e posição.

\subsection{O Limite Clássico}

Vejamos agora a essência do teorema do limite semi-clássico provado por Hepp [21], fazendo uma forte analogia entre as motivações apresentadas por Hepp e o caso presente.

Primeiro, vejamos o significado de $F_{\alpha}(f)$ definido na seção anterior:

$$
\begin{gathered}
F_{\alpha}(f)=\frac{1}{\sqrt{2}}\left(\left\langle f_{1}, \alpha\right\rangle+\left\langle f_{1}, \bar{\alpha}\right\rangle+i\left\langle f_{0}, \alpha\right\rangle-i\left\langle f_{0}, \bar{\alpha}\right\rangle\right)=\left\langle f_{1}, \phi\right\rangle-\left\langle f_{0}, \pi\right\rangle \\
=\left\langle\rho_{1} G f, u_{0}\right\rangle-\left\langle\rho_{0} G f, u_{1}\right\rangle=\langle f, u\rangle .
\end{gathered}
$$

E lembramos, é claro, que $\alpha$ é escrito em termos de $\phi$ e $\pi$, que são a posição e o momento da solução de Klein-Gordon, ou seja, eles são os dados $\left(u_{0}, u_{1}\right)$ do problema de Cauchy

$$
\left\{\begin{array}{l}
\left(\square+m^{2}\right) u=0 \\
\rho_{0}(u)=u_{0} \\
\rho_{1}(u)=u_{1}
\end{array}\right.
$$

Em particular $F_{\alpha}(f)=l(f)$.

O fato que inspira o teorema de Hepp é o seguinte limite: valores esperados de $\Phi(f)$ em estados coerentes centrados em torno de $\hbar^{-1 / 2} \alpha$ geram soluções 
clássicas (não quânticas) da equação de Klein-Gordon. Esse fato é trivial para o caso estático, o teorema de Hepp consiste em ampliar esse resultado para valores esperados evoluídos.

Vejamos a demonstração para o caso que desejamos: devemos provar as seguintes igualdades:

$$
\begin{gathered}
\left\langle 0\left|W\left(\hbar_{\lambda}^{-1 / 2} \alpha\right)^{*}\left[\Phi(f)-\hbar_{\lambda}^{-1 / 2} u(f)\right] W\left(\hbar_{\lambda}^{-1 / 2} \alpha\right)\right| 0\right\rangle=\langle 0|\Phi(f)| 0\rangle, \\
\lim _{\lambda \rightarrow 0}\left\langle 0\left|W\left(\hbar_{\lambda}^{-1 / 2} \alpha\right)^{*} \Phi_{\lambda}(f) W\left(\hbar_{\lambda}^{-1 / 2} \alpha\right)\right| 0\right\rangle=u(f),
\end{gathered}
$$

onde $u(f)=\langle f, u\rangle, \hbar_{\lambda}=\lambda \hbar$, onde $\lambda \in \mathbb{R}$ é o parâmetro que será usado para tomar o limite $\lambda \rightarrow 0$. E, por fim, $\Phi_{\lambda}(f)=\sqrt{\hbar_{\lambda}} \Phi(f)=\sqrt{\lambda \hbar} \Phi(f)$.

A primeira igualdade implica na segunda: por economia chamemos $W\left(\hbar_{\lambda}^{-1 / 2} \alpha\right)|0\rangle=$ $\left|\alpha_{\lambda}\right\rangle$ :

$$
\begin{gathered}
\left\langle\alpha_{\lambda}\left|\Phi(f)-\hbar_{\lambda}^{-1 / 2} u(f)\right| \alpha_{\lambda}\right\rangle=\langle\Phi(f)\rangle_{0}=\hbar_{\lambda}^{-1 / 2}\left\langle\alpha_{\lambda}\left|\hbar_{\lambda}^{1 / 2} \Phi(f)-u(f)\right| \alpha_{\lambda}\right\rangle \\
\Rightarrow\left\langle\alpha_{\lambda}\left|\Phi_{\lambda}(f)\right| \alpha_{\lambda}\right\rangle=\hbar_{\lambda}^{1 / 2}\langle\Phi(f)\rangle_{0}+u(f) \rightarrow u(f) .
\end{gathered}
$$

Basta, então, provar a primeira igualdade. Mas a prova é simples: basta notar que $F_{k \alpha}(f)=k F_{\alpha}(f)$, onde $k$ é um número real arbitrário. Então,

$$
\begin{gathered}
\left\langle\alpha_{\lambda}\left|\Phi(f)-\hbar_{\lambda}^{-1 / 2} u(f)\right| \alpha_{\lambda}\right\rangle=\left\langle\alpha_{\lambda}|\Phi(f)| \alpha_{\lambda}\right\rangle-\hbar_{\lambda}^{-1 / 2} u(f) \\
=\langle 0|\Phi(f)| 0\rangle+\hbar_{\lambda}^{-1 / 2} u(f)-\hbar_{\lambda}^{-1 / 2} u(f) .
\end{gathered}
$$

O teorema de Hepp pretende mostrar que para um operador evoluído, digamos, por algum automorfismo $\mathfrak{a}_{t}, \mathfrak{a}_{t}(\Phi(f))=\Phi(f, t)$ o resultado do limite semi-clássico deve fornecer uma solução de Klein-Gordon evoluída também:

$$
\lim _{\lambda \rightarrow 0}\left\langle\alpha_{\lambda}\left|\mathfrak{a}_{t}\left(\Phi_{\lambda}(f)\right)\right| \alpha_{\lambda}\right\rangle=u(f, t) .
$$

Como é sempre desejável esquivar os problemas advindos de operadores nãolimitados esse limite será estabelecido com a exponencial de $\Phi$ :

$$
\lim _{\lambda \rightarrow 0}\left\langle\alpha_{\lambda}\left|\mathfrak{a}_{t}\left(e^{i \Phi_{\lambda}(f)}\right)\right| \alpha_{\lambda}\right\rangle=e^{i u(f, t)} .
$$

Mas claro, essas afirmações devem ser elaboradas e tornadas precisas (vide 
infra).

Para clarear as ideias que estão por trás do teorema no contexto que nos interessa, prová-lo-emos nas circunstancias da mecânica quântica em uma dimensão: seja o sistema clássico com o Hamiltoniano num espaço de fases $(\pi, \xi) \in \mathbb{R}^{2}, \mathcal{H}(\pi, \xi)=\pi^{2} / 2 m+V(\xi)$. Se $V^{\prime}$ for Lipschitz, as equações de Hamilton,

$$
\left\{\begin{array}{l}
m \dot{\xi}(t)=\pi(t) \\
\dot{\pi}(t)=-V^{\prime}(\xi(t))
\end{array}\right.
$$

possuem uma única solução $(\pi(\alpha, t), \xi(\alpha, t))$ em tempos finitos (possivelmente infinitos também). O que se pretende é comparar essas soluções com as posições e momentos, $q=x, p=-i d / d x$ de um sistema quântico regido pelo Hamiltoniano

$$
H_{\lambda}=-\frac{\hbar_{\lambda}}{2 m} \frac{d^{2}}{d x^{2}}+V\left(\sqrt{\hbar_{\lambda}} x\right) .
$$

Chamaremos também de $H_{\lambda}$ a extensão auto-adjunta do Hamiltoniano acima, e definimos $U_{\lambda}(t)=\exp \left[-i t H_{\lambda} / h_{\lambda}\right]$.

É claro que dado $a=(q+i p) / \sqrt{2}$, o operador $U(\alpha)=\exp \left[\alpha a^{*}-\bar{\alpha} a\right]$ define também um operador de deslocamento: $U(\alpha) a U(\alpha)^{*}=a-\alpha$, e então os estados $|\alpha\rangle=U(\alpha)|0\rangle$ são estados coerentes. Também é fácil de ver (e para isso basta repetir as contas que foram feitas acima) que teremos análogos das igualdades (22) e (23):

$$
\begin{gathered}
\left\langle\hbar_{\lambda}^{-1 / 2} \alpha\left|\left(q-\hbar_{\lambda}^{-1 / 2} \xi\right)\left(p-\hbar_{\lambda}^{-1 / 2} \pi\right)\right| \hbar_{\lambda}^{-1 / 2} \alpha\right\rangle=\langle 0|q p| 0\rangle, \\
\lim _{\lambda \rightarrow 0}\left\langle\hbar_{\lambda}^{-1 / 2} \alpha\left|q_{\lambda} p_{\lambda}\right| \hbar_{\lambda}^{-1 / 2} \alpha\right\rangle=\xi \pi,
\end{gathered}
$$

onde $q_{\lambda}=\sqrt{\hbar_{\lambda}} q$ e $p_{\lambda}=\sqrt{\hbar_{\lambda}} p$. E a essência de um limite clássico que relaciona os dois sistemas é expressa pelo limite $\lim _{\lambda \rightarrow 0}\left\langle\hbar_{\lambda}^{-1 / 2} \alpha\left|q_{\lambda}(t) p_{\lambda}(t)\right| \hbar_{\lambda}^{-1 / 2} \alpha\right\rangle=$ $\xi(\alpha, t) \pi(\alpha, t)$. Mas para evitar problemas relacionados à não-limitabilidade de $p$ e $q$ consideraremos suas exponenciais - como em verdade foi feito em todo esse texto. O teorema a ser provado é o seguinte ([21]):

Teorema 5.1. Sejam $(\xi(\alpha, t), \pi(\alpha, t))=\left(\xi_{t}, \pi_{t}\right)$ soluções de (24) para $|t|<$ T. E seja o potencial $V$ do Hamiltoniano $\mathcal{H}(\xi, \pi)=\pi^{2} / 2 m+V(\xi)$ real, $C^{\delta+2}, \delta>0$ em uma vizinhança de $\xi_{t}$ e tal que $\int|V(x)|^{2} e^{-\rho x^{2}} d x<\infty$, para algum $\rho$ finito. Por fim, seja $H_{\lambda}$ a extensão auto-adjunta de (25) e $U_{\lambda}(t)=\exp \left(-i H_{\lambda} t / \hbar_{\lambda}\right)$. Então,

$$
\begin{array}{r}
s-\lim _{\lambda \rightarrow 0} U\left(\hbar_{\lambda}^{-1 / 2} \alpha\right)^{*} U_{\lambda}(t)^{*} e^{i\left[r\left(q-\hbar_{\lambda}^{-1 / 2} \xi_{t}\right)+s\left(p-\hbar_{\lambda}^{-1 / 2} \pi_{t}\right)\right]} U_{\lambda}(t) U\left(\hbar_{\lambda}^{-1 / 2} \alpha\right) \\
=e^{i[r q(\alpha, t)+s p(\alpha, t)]}
\end{array}
$$


$E$

$$
s-\lim _{\lambda \rightarrow 0} U\left(\hbar_{\lambda}^{-1 / 2} \alpha\right)^{*} U_{\lambda}(t)^{*} e^{i\left[r q_{\lambda}+s p_{\lambda}\right]} U_{\lambda}(t) U\left(\hbar_{\lambda}^{-1 / 2} \alpha\right)=e^{i\left[r \xi_{t}+s \pi_{t}\right]},
$$

onde $q(\alpha, t)$ e $p(\alpha, t)$ são soluções de (24) com o potencial linearizado em torno de $\xi_{t}: H(t)=p^{2} / 2 m+V^{\prime \prime}\left(\xi_{t}\right) q^{2} / 2$.

Prova: A prova pode ser dividida em duas partes principais: a primeira escrevemos o lado esquerdo de (26) da forma $W_{\lambda}(t, 0)^{*} \exp (i[r q+s p]) W_{\lambda}(t, 0)$, para algum operador $W_{\lambda}$ unitário tal que

$$
\mathrm{s}-\lim _{\lambda \rightarrow 0} W_{\lambda}(t, s)=T \exp \left(-i \int_{s}^{t} H(r) d r\right)=: W(t, s),
$$

onde $T$ é o operador de ordenação temporal. Se esse for o caso é imediato que (26) segue, e veremos que (27) também segue facilmente. A segunda parte consiste apenas em mostrar que de fato o $W_{\lambda}(t, s)$ definido converge fortemente à $W(t, s)$.

Primeiro expandimos $H_{\lambda} / \hbar_{\lambda}$ em torno de $\xi_{t}, H_{\lambda} / \hbar_{\lambda}=H_{\lambda}^{0}(t)+H_{\lambda}^{1}(t)+H_{\lambda}^{2}(t)+$ ..., onde

$$
\begin{gathered}
H_{\lambda}^{0}(t)=\mathcal{H}(\xi, \pi) / \hbar_{\lambda} \\
H_{\lambda}^{1}(t)=\pi_{t}\left(p-\hbar_{\lambda}^{-1 / 2} \pi_{t}\right) \hbar_{\lambda}^{-1 / 2} / m+V^{\prime}\left(\xi_{t}\right)\left(q-\hbar_{\lambda}^{-1 / 2} \xi_{t}\right) \hbar_{\lambda}^{-1 / 2}, \\
H_{\lambda}^{2}(t)=\left(p-\hbar_{\lambda}^{-1 / 2} \pi_{t}\right)^{2} / 2 m+\frac{V^{\prime \prime}\left(\xi_{t}\right)}{2}\left(q-\hbar_{\lambda}^{-1 / 2} \xi_{t}\right)^{2} .
\end{gathered}
$$

Seja o propagador $U_{\lambda}^{1}(t)=T \exp \left(-i \int_{0}^{t} H_{\lambda}^{1}(r) d r\right)$, que existe para todo $|t|<T$ e satisfaz:

$$
\begin{aligned}
& U_{\lambda}^{1}(t)^{*}\left(a-\hbar_{\lambda}^{-1 / 2} \alpha_{t}\right) U_{\lambda}^{1}(t)=a-\hbar_{\lambda}^{-1 / 2} \alpha, \quad U_{\lambda}^{1}(t)^{*}\left(a^{*}-\hbar_{\lambda}^{-1 / 2} \bar{\alpha}_{t}\right) U_{\lambda}^{1}(t)= \\
& =a^{*}-\hbar_{\lambda}^{-1 / 2} \bar{\alpha} \text {. }
\end{aligned}
$$

Para provar isso, tomemos $A=a-\hbar_{\lambda}^{-1 / 2} \alpha_{t}$ e $B=i \int_{0}^{t} H_{\lambda}^{1}(s) d s$ (e então $\left.B^{*}=-B\right)$. Queremos calcular $\left(T e^{-B}\right)^{*} A\left(T e^{-B}\right)$. Como os operadores $p$ e $q$ em $H_{\lambda}^{1}$ não dependem do tempo a ordenação temporal pode ser ignorada, e segundo a série de Lie (vide, e.g., Cap. 9 de [4]):

$$
\left.\left.e^{B} A e^{-B}=A+\sum_{n=1}^{\infty} \frac{1}{n !}[\overbrace{B,[B, \ldots,[B}^{n}, A] \ldots\right]\right] .
$$

Importa, então, saber uma forma mais explícita de $B$. Vejamos:

$$
H_{\lambda}^{1}(s)=\pi_{s}\left(p-\hbar_{\lambda}^{-1 / 2} \pi_{s}\right) \hbar_{\lambda}^{-1 / 2} / m+V^{\prime}\left(\xi_{s}\right)\left(q-\hbar_{\lambda}^{-1 / 2} \xi_{s}\right) \hbar_{\lambda}^{-1 / 2}=
$$




$$
=\hbar_{\lambda}^{-1 / 2} \pi_{s} p / m+\hbar_{\lambda}^{-1 / 2} V^{\prime}\left(\xi_{s}\right) q+f \mathbb{1},
$$

onde $f$ é um número, e assim não influenciará no comutador $[B, A]$. Então,

$$
\begin{aligned}
i \int_{0}^{t} H_{\lambda}^{1}(s) d s= & i(\hbar_{\lambda}^{-1 / 2}(p / m) \int_{0}^{t} \overbrace{\pi_{s}}^{=m \dot{\xi}_{s}} d s+\hbar_{\lambda}^{-1 / 2} q \int_{0}^{t} \overbrace{V^{\prime}\left(\xi_{s}\right)}^{=-\dot{\pi}_{s}} d s+\overbrace{\int \frac{1}{f d s}}^{=C})= \\
& =i \hbar_{\lambda}^{-1 / 2} \underbrace{\left(\xi_{t}-\xi_{0}\right)}_{=\Delta \xi} p-i \hbar_{\lambda}^{-1 / 2} \underbrace{\left(\pi_{t}-\pi_{0}\right)}_{=\Delta \pi} q+i C \mathbb{1} .
\end{aligned}
$$

Então,

$$
\begin{gathered}
{[B, A]=\frac{i \hbar_{\lambda}^{-1 / 2}}{\sqrt{2}}[\Delta \xi p-\Delta \pi q, q+i p]=\frac{i \hbar_{\lambda}^{-1 / 2}}{\sqrt{2}}(\Delta \xi[p, q]-\Delta \pi(i[q, p]))} \\
=\frac{\hbar_{\lambda}^{-1 / 2}}{\sqrt{2}}(\Delta \xi+i \Delta \pi)=\frac{\hbar_{\lambda}^{-1 / 2}}{\sqrt{2}}\left(\xi_{t}-\xi+\pi_{t}-\pi\right)=\hbar_{\lambda}^{-1 / 2} \alpha_{t}-\hbar_{\lambda}^{-1 / 2} \alpha .
\end{gathered}
$$

Analogamente para $A^{*}=a^{*}-\hbar_{\lambda}^{-1 / 2} \bar{\alpha}_{t}$.

Com esses propagadores definimos

$$
W_{\lambda}(t, s)=U\left(\hbar_{\lambda}^{-1 / 2} \alpha\right)^{*} U_{\lambda}^{1}(t)^{*} U_{\lambda}(t-s) U_{\lambda}^{1}(s) U\left(\hbar_{\lambda}^{-1 / 2} \alpha\right) e^{i \int_{s}^{t} H_{\lambda}^{0}(r) d r} .
$$

Então o lado esquerdo de (26) pode ser escrito como

$$
W_{\lambda}(t, 0)^{*} e^{i[r q+s p]} W_{\lambda}(t, 0) .
$$

Onde, claro,

$$
W_{\lambda}(t, 0)=U\left(\hbar_{\lambda}^{-1 / 2} \alpha\right)^{*} U_{\lambda}^{1}(t)^{*} U_{\lambda}(t) U\left(\hbar_{\lambda}^{-1 / 2} \alpha\right) e^{i \int_{0}^{t} H_{\lambda}^{0}(r) d r} .
$$

Ou seja, devemos mostrar que

$$
\begin{aligned}
& W_{\lambda}(t, 0)^{*} e^{i[r q+s p]} W_{\lambda}(t, 0)= \\
& \quad=U\left(\hbar_{\lambda}^{-1 / 2} \alpha\right)^{*} U_{\lambda}(t)^{*} e^{i[r q+s p]-i \hbar_{\lambda}^{-1 / 2}\left[r \xi_{t}-s \pi_{t}\right]} U_{\lambda}(t) U\left(\hbar_{\lambda}^{-1 / 2} \alpha\right) .
\end{aligned}
$$

Mas

$$
\begin{gathered}
W_{\lambda}(t, 0)^{*} e^{i[r q+s p]} W_{\lambda}(t, 0)= \\
=U\left(\hbar_{\lambda}^{-1 / 2} \alpha\right)^{*} U_{\lambda}(t)^{*} \underbrace{U_{\lambda}^{1}(t) U\left(\hbar_{\lambda}^{-1 / 2} \alpha\right) e^{i[r q+s p]} U\left(\hbar_{\lambda}^{-1 / 2} \alpha\right) U_{\lambda}^{1}(t)}_{=\mathcal{W}} U_{\lambda}(t) U\left(\hbar_{\lambda}^{-1 / 2} \alpha\right) .
\end{gathered}
$$


Então, deve ser verdade que $\mathcal{W}=\exp \left(i[r q+s p]-i \hbar_{\lambda}^{-1 / 2}\left[r \xi_{t}-s \pi_{t}\right]\right)$, e de fato isso é verdade. Vejamos:

$r q+s p=\frac{1}{\sqrt{2}}\left[r a+r a^{*}-i s a+i s a^{*}\right]=\frac{1}{\sqrt{2}}\left[(r-i s) a+(r+i s) a^{*}\right]=\bar{z} a+z a^{*}$,

onde $z=(r+i s) / \sqrt{2}$. Então,

$$
\begin{aligned}
& U\left(\hbar_{\lambda}^{-1 / 2} \alpha\right) \exp \left(i\left[\bar{z} a+z a^{*}\right]\right) U\left(\hbar_{\lambda}^{-1 / 2} \alpha\right)^{*}= \\
& =U\left(\hbar_{\lambda}^{-1 / 2} \alpha\right)\left[\mathbb{1}+i\left(\bar{z} a+z a^{*}\right)+\frac{i^{2}}{2}\left(\bar{z} a+z a^{*}\right)^{2}+\cdots\right] U\left(\hbar_{\lambda}^{-1 / 2} \alpha\right)^{*}= \\
& =\mathbb{1}+i\left[\bar{z}\left(a-\hbar_{\lambda}^{-1 / 2} \alpha\right)+z\left(a^{*}-\hbar_{\lambda}^{-1 / 2} \bar{\alpha}\right)\right]+ \\
& +\frac{i^{2}}{2 !} U\left(\hbar_{\lambda}^{-1 / 2} \alpha\right)\left(\bar{z} a+z a^{*}\right) U\left(\hbar_{\lambda}^{-1 / 2} \alpha\right)^{*} U\left(\hbar_{\lambda}^{-1 / 2} \alpha\right)\left(\bar{z} a+z a^{*}\right) U\left(\hbar_{\lambda}^{-1 / 2} \alpha\right)^{*}+\cdots \\
& =\mathbb{1}+i\left[\bar{z}\left(a-\hbar_{\lambda}^{-1 / 2} \alpha\right)+z\left(a^{*}-\hbar_{\lambda} r^{-1 / 2} \bar{\alpha}\right)\right]+\frac{i^{2}}{2 !}\left(\bar{z}\left(a-\hbar_{\lambda}^{-1 / 2} \alpha\right)+z\left(a^{*}-\hbar_{\lambda}^{-1 / 2} \bar{\alpha}\right)\right)^{2}+\cdots
\end{aligned}
$$

Agora aplicando $U_{\lambda}^{1}(t)$ :

$$
\begin{aligned}
& \mathcal{W}=U_{\lambda}^{1}(t)\left(\mathbb{1}+i\left[\bar{z}\left(a-\hbar_{\lambda}^{-1 / 2} \alpha\right)+z\left(a^{*}-\hbar_{\lambda}^{-1 / 2} \bar{\alpha}\right)\right]+\right. \\
& \left.+\frac{i^{2}}{2 !}\left(\bar{z}\left(a-\hbar_{\lambda}^{-1 / 2} \alpha\right)+z\left(a^{*}-\hbar_{\lambda}^{-1 / 2} \bar{\alpha}\right)\right)^{2}+\cdots\right) U_{\lambda}^{1}(t)^{*} \\
& =\mathbb{1}+i\left[\bar{z}\left(a-\hbar_{\lambda}^{-1 / 2} \alpha_{t}\right)+z\left(a^{*}-\hbar_{\lambda}^{-1 / 2} \bar{\alpha}_{t}\right)\right]+ \\
& +\frac{i^{2}}{2 !} U_{\lambda}^{1}(t)\left[\bar{z}\left(a-\hbar_{\lambda}^{-1 / 2} \alpha\right)+z\left(a^{*}-\hbar_{\lambda}^{-1 / 2} \bar{\alpha}\right)\right] U_{\lambda}^{1}(t)^{*} U_{\lambda}^{1}(t)\left[\bar{z}\left(a-\hbar_{\lambda}^{-1 / 2} \alpha\right)+\right. \\
& \left.+z\left(a^{*}-\hbar_{\lambda}^{-1 / 2} \bar{\alpha}\right)\right] U_{\lambda}^{1}(t)^{*}+\cdots \\
& =\mathbb{1}+\sum_{n=1}^{\infty} \frac{i^{n}}{n !}\left[\bar{z}\left(a-\hbar_{\lambda}^{-1 / 2} \alpha_{t}\right)+z\left(a^{*}-\hbar_{\lambda}^{-1 / 2} \bar{\alpha}_{t}\right)\right]^{n}= \\
& =\exp \left(i\left[\bar{z}\left(a-\hbar_{\lambda}^{-1 / 2} \alpha_{t}\right)+z\left(a^{*}-\hbar_{\lambda}^{-1 / 2} \bar{\alpha}_{t}\right)\right]\right) .
\end{aligned}
$$

Mas,

$\bar{z} a+z a^{*}-\hbar_{\lambda}^{-1 / 2}\left(\bar{z} \alpha_{t}-z \bar{\alpha}_{t}\right)=r q+s p-\hbar_{\lambda}^{-1 / 2}\left[r\left(\alpha_{t}+\bar{\alpha}_{t}\right)+i s\left(\alpha_{t}-\bar{\alpha}_{t}\right)\right] / \sqrt{2}$.

E como $\alpha_{t}=\left(\xi_{t}+i \pi_{t}\right) / \sqrt{2}$ obtemos $i\left[\bar{z}\left(a-\hbar_{\lambda}^{-1 / 2} \alpha_{t}\right)+z\left(a^{*}-\hbar_{\lambda}^{-1 / 2} \bar{\alpha}_{t}\right)\right]=$ $r q+s p-\hbar_{\lambda}^{-1 / 2}\left[r \xi_{t}-s \pi_{t}\right]$.

Resta-nos agora provar que s-lim $W_{\lambda}(t, s)=W(t, s)$ em um subespaço denso, 
e este subespaço será o seguinte conjunto gerador de $L^{2}(\mathbb{R})$ formado por Gaussianas: $\left\{\psi_{a} \in L^{2}(\mathbb{R}): a \in \mathbb{R}\right\}$, onde $\psi_{a}=\pi^{-1 / 4} \exp \left[-(x-a)^{2} / 2\right]$. Devemos, então, analisar a norma de $W_{\lambda}(t, 0) \psi_{a}-W(t, 0) \psi_{a}$, mas esta diferença pode ser escrita, de acordo com o Teorema Fundamental do Cálculo:

$$
W_{\lambda}(t, 0) \psi_{a}-W(t, 0) \psi_{a}=\int_{0}^{t} \frac{d}{d s} W_{\lambda}(t, s) W(s, 0) \psi_{a} d s .
$$

Importa, então, saber a derivada em $s$ de $W_{\lambda}(t, s) W(s, 0)$, já sabendo que formalmente temos

$$
\begin{gathered}
\frac{d}{d s} U_{\lambda}(t-s)=\frac{i}{\hbar_{\lambda}} H_{\lambda} U_{\lambda}(t-s), \quad \frac{d}{d s} U_{\lambda}^{1}(s)=-i H_{\lambda}^{1}(s) U_{\lambda}^{1}(s), \\
\frac{d}{d s} e^{i \int_{s}^{t} H_{\lambda}^{0}(r) d r}=-i H_{\lambda}^{0}(s) e^{-i \int_{s}^{t} H_{\lambda}^{0}(r) d r}, \quad \frac{d}{d s} W(s, 0)=-i H(s) W(s, 0) .
\end{gathered}
$$

E então,

$$
(d / d s) W_{\lambda}(t, s) W(s, 0) \psi_{a}=D_{1}+D_{2},
$$

onde

$$
D_{2}=W_{\lambda}(s, t)(d / d s) W(s, 0) \psi_{a}=-i W_{\lambda}(s, 0) H(s) W(s, 0) \psi_{a}
$$

e

$$
\begin{gathered}
D_{1}=\frac{d}{d s}\left[U\left(\hbar_{\lambda}^{-1 / 2} \alpha\right)^{*} U_{\lambda}^{1}(t)^{*} U_{\lambda}(t-s) U_{\lambda}^{1}(s) U\left(\hbar_{\lambda}^{-1 / 2} \alpha\right) e^{i \int_{s}^{t} \mathscr{H}(r) / \hbar_{\lambda} d r}\right] W(s, 0) \psi_{a} \\
=U\left(\hbar_{\lambda}^{-1 / 2} \alpha\right)^{*} U_{\lambda}^{1}(t)^{*} e^{i \int_{s}^{t} \mathscr{H}(r) / \hbar_{\lambda} d r} U_{\lambda}(t-s)\left[i \hbar_{\lambda}^{-1} H_{\lambda}(s)-\right. \\
\left.-i H_{\lambda}^{1}(s)-i \hbar_{\lambda}^{-1} \mathscr{H}(s)\right] U_{\lambda}^{1}(s) U\left(\hbar_{\lambda}^{-1 / 2} \alpha\right) W(s, 0) \psi_{a} .
\end{gathered}
$$

Seja $\mathscr{U}:=U\left(\hbar_{\lambda}^{-1 / 2} \alpha\right)^{*} U_{\lambda}^{1}(t)^{*} e^{i \int_{s}^{t} \mathscr{H}(r) / \hbar_{\lambda} d r} U_{\lambda}(t-s)$. Assim,

$$
\begin{aligned}
D_{1} & =i \mathscr{U}\left[\hbar_{\lambda}^{-1}\left(\frac{\hbar_{\lambda}}{2 m} p^{2}+V\left(q_{\lambda}\right)\right)-\hbar_{\lambda}^{-1 / 2} \pi_{s} p-\hbar_{\lambda}^{-1 / 2} V^{\prime}\left(\xi_{s}\right) q+\right. \\
& \left.+\hbar_{\lambda}^{-1} \pi_{s}^{2}+\hbar_{\lambda}^{-1} \xi_{s} V^{\prime}\left(\xi_{s}\right)-\hbar_{\lambda}^{-1}\left(\frac{\pi_{s}^{2}}{2 m}+V\left(\xi_{s}\right)\right)\right] U_{\lambda}^{1}(s) U\left(\hbar_{\lambda}^{-1 / 2} \alpha\right) W(s, 0) \psi_{a} .
\end{aligned}
$$

Dividimos $D_{1}$ em ainda outras duas partes, viz.,

$$
D_{11}=i \mathscr{U} \hbar_{\lambda}^{-1}\left[\frac{\hbar_{\lambda}}{2 m} p^{2}-\frac{\pi_{s}^{2}}{2 m}+V\left(q_{\lambda}\right)-V\left(\xi_{s}\right)\right] \psi_{a}^{\lambda s},
$$




$$
\begin{aligned}
& D_{12}=-i \mathscr{U} \hbar_{\lambda}^{-1 / 2}\left[\pi_{s}\left(p-\hbar_{\lambda}^{-1 / 2} \pi_{s}\right) / m+\right. \\
& \left.\quad+V^{\prime}\left(\xi_{s}\right)\left(q-\hbar_{\lambda}^{-1 / 2} \xi_{s}\right)\right] U_{\lambda}^{1}(s) U\left(\hbar_{\lambda}^{-1 / 2} \alpha\right) W(s, 0) \psi_{a},
\end{aligned}
$$

onde $\psi_{a}^{\lambda s}:=U_{\lambda}^{1}(s) U\left(\hbar_{\lambda}^{-1 / 2} \alpha\right) W(s, 0) \psi_{a}$. Lembrando que $U_{\lambda}^{1}$ e $U$ satisfazem: $U\left(\hbar_{\lambda}^{-1 / 2} \alpha\right) q=\left(q-\hbar_{\lambda}^{-1 / 2} \xi\right) U\left(\hbar_{\lambda}^{-1 / 2} \alpha\right)$ e $U_{\lambda}^{1}(s)\left[q-\hbar_{\lambda}^{-1 / 2} \xi\right]=\left[q-\hbar_{\lambda}^{-1 / 2} \xi_{s}\right] U_{\lambda}^{1}(s)$ (e relações análogas para $p$ ) temos,

$$
D_{12}=-i \hbar_{\lambda}^{-1 / 2} W_{\lambda}(s, t)\left[\pi_{s} p / m+V^{\prime}\left(\xi_{s}\right) q\right] W(s, 0) \psi_{a}
$$

e

$$
\begin{aligned}
D_{11}=i \mathscr{U} \hbar_{\lambda}^{-1}\left[\frac{\hbar_{\lambda}}{2 m}(\overbrace{p^{2}+\left(\hbar_{\lambda}^{-1} \pi_{s}^{2}-2 p \hbar_{\lambda}^{-1 / 2} \pi_{s}\right)}^{=\left(p-\hbar_{\lambda}^{-1 / 2} \pi_{s}\right)^{2}}-\left(\hbar_{\lambda}^{-1} \pi_{s}^{2}-2 p \hbar_{\lambda}^{-1 / 2} \pi_{s}\right))+\right. \\
\left.+\frac{\pi_{s}^{2}}{2 m}+\mathscr{V}\right] \psi_{a}^{\lambda s},
\end{aligned}
$$

onde $\mathscr{V}=V\left(q_{\lambda}\right)-V\left(\xi_{s}\right)$. Então:

$$
D_{11}=i W_{\lambda}(t, s) \frac{p^{2}}{2 m} W(s, 0) \psi_{a}+A+i \mathscr{U} \hbar_{\lambda}^{-1} \mathscr{V} \psi_{a}^{\lambda s},
$$

onde,

$$
\begin{gathered}
A=i \mathscr{U} \hbar_{\lambda}^{-1}\left[-\frac{\pi_{s}^{2}}{m}+\frac{1}{m} \hbar_{\lambda}^{1 / 2} \pi_{s} p\right] \psi_{a}^{\lambda s}=i \mathscr{U} \frac{\hbar_{\lambda}^{-1 / 2}}{m} \pi_{s}\left[p-\hbar_{\lambda}^{-1 / 2} \pi_{s}\right] \psi_{a}^{\lambda s} \\
=i \frac{\hbar_{\lambda}^{-1 / 2}}{m} \pi_{s} W(t, s) p W(s, 0) \psi_{a} .
\end{gathered}
$$

Para passar $U_{\lambda}^{1}(s) U\left(\hbar_{\lambda}^{-1 / 2} \alpha\right)$ à esquerda de $V\left(q_{\lambda}\right)$ expandimos $V\left(q_{\lambda}\right)$ em torno de $\hbar_{\lambda}^{-1 / 2} \xi_{s}$ :

$$
V\left(q_{\lambda}\right)=\sum V_{n}\left(\hbar_{\lambda}^{-1 / 2} \xi_{s}\right)\left(q_{\lambda}-\hbar_{\lambda}^{-1 / 2} \xi_{s}\right)^{n}
$$

e como $\left(q_{\lambda}-\hbar_{\lambda}^{-1 / 2} \xi_{s}\right)^{n} U_{\lambda}^{1}(s) U\left(\hbar_{\lambda}^{-1 / 2} \alpha\right)=U_{\lambda}^{1}(s) U\left(\hbar_{\lambda}^{-1 / 2} \alpha\right) q_{\lambda}^{n}$,

$$
V\left(q_{\lambda}\right) U_{\lambda}^{1}(s) U\left(\hbar_{\lambda}^{-1 / 2} \alpha\right)=U_{\lambda}^{1}(s) U\left(\hbar_{\lambda}^{-1 / 2} \alpha\right) \sum V_{n}\left(\hbar_{\lambda}^{-1 / 2} \xi_{s}\right) q_{\lambda}^{n} .
$$

A somatória é a expansão de $V\left(\hbar_{\lambda}^{1 / 2}\left(q+\hbar_{\lambda}^{-1 / 2} \xi_{s}\right)\right)$ em torno de $\hbar_{\lambda}^{-1 / 2} \xi_{s}$, e $\operatorname{assim} V\left(q_{\lambda}\right) U_{\lambda}^{1}(s) U\left(\hbar_{\lambda}^{-1 / 2} \alpha\right)=U_{\lambda}^{1}(s) U\left(\hbar_{\lambda}^{-1 / 2} \alpha\right) V\left(q_{\lambda}+\xi_{s}\right)$.

E portanto,

$$
D_{11}=i W(t, s)\left[\frac{p^{2}}{2 m}+\frac{\hbar_{\lambda}^{-1 / 2}}{m} \pi_{s} p+\hbar_{\lambda}^{-1} V\left(q_{\lambda}+\xi_{s}\right)-\hbar_{\lambda}^{-1} V\left(\xi_{s}\right)\right] W(s, 0) \psi_{a}
$$

\footnotetext{
${ }^{49} \mathrm{E}$ ainda, introduzindo $\mathbb{1}=U^{*} U=\left(U_{\lambda}^{1}\right)^{*} U_{\lambda}^{1}$ obtemos relações similares para $q^{2}$ e $p^{2}$.
} 
Temos, portanto,

$$
\begin{aligned}
& \frac{d}{d s} W_{\lambda}(t, s) W(s, 0) \psi_{a}= \\
& =i W_{\lambda}(t, s)\left[\hbar_{\lambda}^{-1} V\left(q_{\lambda}+\xi_{s}\right)-\right. \\
& \left.-\hbar_{\lambda}^{-1} V\left(\xi_{s}\right)-\hbar_{\lambda}^{-1 / 2} V^{\prime}\left(\xi_{s}\right) q+V^{\prime \prime}\left(\xi_{s}\right) \frac{q^{2}}{2}\right] W(s, 0) \psi_{a} .
\end{aligned}
$$

Para que $D_{1}$ e $D_{2}$ estejam bem definidos temos que saber a forma de $\psi_{a}^{\lambda s}:=$ $U_{\lambda}^{1}(s) U\left(\hbar_{\lambda}^{-1 / 2} \alpha\right) W(s, 0) \psi_{a}$, em particular, devemos provar que existe um $\hbar_{k}$ tal que para todo $\lambda$ tal que $\hbar_{\lambda}<\hbar_{k}$, temos $\left\{\psi_{a}^{\lambda s}\right\} \subset D\left(p^{2}\right) \cap D\left(\hbar_{\lambda}^{-1} V\left(q_{\lambda}\right)\right)^{50}$. Ou seja, a partir de um mínimo $\hbar_{k}, \psi_{a}^{\lambda s}$ está bem definido e as contas acima valem.

Primeiro:

$$
W(s, 0) q W(s, 0)^{*}=\alpha q+\beta p, \quad W(s, 0) p W(s, 0)^{*}=\gamma q+\delta p,
$$

E os coeficientes $\alpha, \beta, \gamma, \delta$, todos dependentes de $s$, formam uma matriz que pertence ao grupo de matrizes simpléticas:

$$
\left(\begin{array}{cc}
\alpha & \beta \\
\gamma & \delta
\end{array}\right) \in S p(2, \mathbb{R}), \quad \text { i.e., } \quad \alpha \delta-\beta \gamma=1 .
$$

Para provar isto usamos novamente a série de Lie (28), onde $e^{B}=W(s, 0)=$ $\exp \left(i \int_{0}^{s} H(r) d r\right)$. Novamente a ordenação temporal não importa aqui já que $p$ e $q$ não dependem do tempo. Como $H(r)=p^{2} / 2 m+V^{\prime \prime}\left(\xi_{r}\right) q^{2} / 2$,

$$
B=i\left(\frac{p^{2} s}{2 m}+\frac{q^{2} f(s)}{2}\right)
$$

onde $f(s)=\int_{0}^{s} V^{\prime \prime}\left(\xi_{r}\right) d r$. Então,

$$
\begin{aligned}
& {[B, q]=i\left[\frac{p^{2} s}{2 m}+\frac{q^{2} f(s)}{2}, q\right]=-i\left[q, \frac{s p^{2}}{2 m}\right]=-\frac{i s}{2 m}([q, p] p+p[q, p])=\frac{s p}{m}} \\
& \frac{1}{2 !}[B,[B, q]]=\frac{1}{2 !} \frac{s}{m}[B, p]=\frac{1}{2 !} \frac{s f(s)}{m} q, \quad \frac{1}{3 !}[B,[B,[B, q]]]=\frac{1}{3 !} \frac{s^{2} f(s) p}{m^{2}} .
\end{aligned}
$$

Ou seja,

$$
\left.\left.e^{B} q e^{-B}=q+\sum_{n=1}^{\infty} \frac{1}{n !}[\overbrace{B,[B, \ldots,[B}^{n}, q] \ldots\right]\right]
$$

\footnotetext{
${ }^{50}$ Essa condição basta também para garantir $D_{2}$, já que se $\psi_{a}^{\lambda s} \in D\left(p^{2}\right), \psi_{a}^{\lambda s} \in D\left(q^{2}\right)$.
} 
é da forma $\alpha(s) q+\beta(s) q$, onde $\alpha(s)$ e $\beta(s)$ são somatórias de alguma potência de $s f(s) / m$ por $n$ !. É certo, então, que se tivéssemos considerado $W(s, t)$ ao invés de $W(s, 0)$ essas somatórias convergiriam se $|s-t|$ fosse pequeno. Da mesma forma, $\alpha(s)$ e $\beta(s)$ convergem e estão bem definidas para $|s| \leq k$, para algum $k<T$. Da mesma forma estimamos $W(s, 0) p W(s, 0)^{*}$.

E como $i p e^{-(x-a)^{2} / 2}=(d / d x) e^{-(x-a)^{2} / 2}=(-x+a) e^{-(x-a)^{2} / 2},(q+i p-a) \psi_{a}=$ 0 :

$$
\begin{gathered}
0=U_{\lambda}^{1}(s) U\left(\hbar_{\lambda}^{-1 / 2} \alpha\right) W(s, 0)(q+i p-a) \psi_{a} \\
=U_{\lambda}^{1}(s) U\left(\hbar_{\lambda}^{-1 / 2} \alpha\right)[(\alpha+i \gamma) q+(\beta+i \delta) p-a] W(s, 0) \psi_{a} \\
=\left[(\alpha+i \gamma)\left(q-\hbar_{\lambda}^{-1 / 2} \xi_{s}\right)+i(\delta-i \beta)\left(p-\hbar_{\lambda}^{-1 / 2} \pi_{s}\right)-a\right] \psi_{a}^{\lambda s}=0,
\end{gathered}
$$

então, $\psi_{a}^{\lambda s}$ satisfaz a seguinte equação:

$$
\left[(\alpha+i \gamma) x+(\delta-i \beta) \frac{d}{d x}-A\right] \psi_{a}^{\lambda s}=0
$$

onde, $A=(\alpha+i \gamma) \hbar_{\lambda}^{-1 / 2} \xi_{s}+i(\delta-i \beta) \hbar_{\lambda}^{-1 / 2} \pi_{s}+a$. E assim $\psi_{a}^{\lambda s}$ é da forma $\exp \left[k_{1} x^{2}+k_{2} x\right]$. Desta forma concluímos que $\psi_{a}^{\lambda s} \in D\left(p^{2}\right)$

Substituindo este Ansatz na equação acima obtemos as condições sobre $k_{1} \mathrm{e}$ $k_{2}$ :

$$
2 k_{1} x+k_{2}=-\frac{(\alpha+i \gamma)}{\delta-i \beta}+\frac{A}{\delta-i \beta} \Rightarrow\left\{\begin{array}{l}
k_{1}=-\frac{(\alpha+i \gamma)}{2(\delta-i \beta)} \\
k_{2}=\frac{A}{\delta-i \beta}
\end{array} .\right.
$$

Como queremos saber se $\psi_{a}^{\lambda s} \in D\left(\hbar_{\lambda}^{-1} V\left(q_{\lambda}\right)\right)$ devemos verificar que

$$
\int \hbar_{\lambda}^{-2}\left|V\left(q_{\lambda}\right)\right|^{2}\left|\psi_{a}^{\lambda s}\right|^{2} d x<\infty
$$

Assim, importa apenas a parte real do argumento da exponencial que é $\psi_{a}^{\lambda s}$ :

$$
\begin{aligned}
\psi_{a}^{\lambda s}(x)=k \exp [ & \left.-\Re\left(\frac{\alpha+i \delta}{2(\delta-i \beta)}\right) x^{2}+\Re\left(\frac{A}{\delta-i \beta}\right) x\right]= \\
= & k \exp \left[-\frac{1}{2\left(\delta^{2}+\beta^{2}\right)}\left(x^{2}-2 \Re(A) \delta x\right)\right],
\end{aligned}
$$

onde $k$ é uma constante; e no cálculo utilizamos o fato de que como $\alpha \delta-\gamma \beta=$ $1, \Re((\alpha-i \gamma) /(\delta-i \beta))=1 /\left(\delta^{2}+\beta^{2}\right)$. É claro que podemos completar o quadrado na exponencial de $\psi_{a}^{\lambda s}$ e incorporar a constante que aparecerá em $k$, de modo que $\psi_{a}^{\lambda s}$ é:

$$
\psi_{a}^{\lambda s}=k \exp \left(-\frac{x^{2}}{2\left(\delta^{2}+\beta^{2}\right)}\right)
$$


Como $\delta$ e $\beta$ convergem, para todo $s$ em $|s| \leq k$, existe um $\eta_{k}>0$ tal que $1 / 2\left(\delta^{2}+\beta^{2}\right)>\eta_{k}$. Como é de hipótese que $\int|V(x)|^{2} \exp \left(-\rho x^{2}\right) d x<\infty$, se tomarmos $\hbar_{k}=\eta_{k} / \rho$ fica provado que $\psi_{a}^{\lambda s} \in D\left(\hbar_{\lambda}^{-1} V\left(q_{\lambda}\right)\right)$, já que se de fato $\hbar_{\lambda}<\hbar_{k}$,

$$
\begin{array}{r}
\int\left|V\left(x_{\lambda}\right)\right|^{2}\left|\psi_{a}^{\lambda s}\right|^{2} d x=\int\left|V\left(x_{\lambda}\right)\right|^{2} \exp \left(-\frac{x^{2}}{2\left(\delta^{2}+\beta^{2}\right)}\right) d x \\
<\int\left|V\left(x_{k}\right)\right|^{2} \exp \left(-x^{2} \eta_{k}\right) d x
\end{array}
$$

Onde $x_{k}=\sqrt{\hbar_{k}} x$. Com a mudança de variável $x \mapsto \hbar_{k}^{-1 / 2} x$ obtemos precisamente $\int|V(x)|^{2} \exp \left(-\rho x^{2}\right) d x$, que é menor do que infinito.

Voltando agora às expressões (29) e (30):

$$
\begin{gathered}
\left\|W_{\lambda}(t, 0) \psi_{a}-W(t, 0) \psi_{a}\right\|^{2}=\left\|\int_{0}^{t} \frac{d}{d s} \psi_{a}^{\lambda s} d s\right\|^{2}=\int_{\mathbb{R}^{2}}\left|\left(\int_{0}^{t} \frac{d}{d s} \psi_{a}^{\lambda s} d s\right)(x)\right|^{2} d x \\
\leq \iint_{0}^{t}\left|\left(\frac{d}{d s} \psi_{a}^{\lambda s}\right)(x)\right|^{2} d s d x .
\end{gathered}
$$

Consideramos, então, a integral em $x$ :

$\int\left|\hbar_{\lambda}^{-1} V\left(x_{\lambda}+\xi_{s}\right)-\hbar_{\lambda}^{-1} V\left(\xi_{s}\right)-\hbar_{\lambda}^{-1 / 2} V^{\prime}\left(\xi_{s}\right) x-V^{\prime \prime}\left(\xi_{s}\right) \frac{x^{2}}{2}\right|^{2}\left|\left(W(s, 0) \psi_{a}\right)(x)\right|^{2} d x$.

Vejamos $x_{\lambda}$ em uma vizinhança de $\xi_{s}$ e fora desta vizinhança: para $x_{\lambda}$ em uma vizinhança de $\xi_{s}$ existe um $\sigma$ tal que para todo $x$ em $\left\{|x| \leq \hbar_{\lambda}^{-1 / 2} \sigma\right\}$, $V\left(x_{\lambda}+\xi_{s}\right)$ é $C^{2+\delta}, \delta>0$.

Fora desta região, $\left|\left(W(s, 0) \psi_{a}\right)(x)\right|^{2}$ decresce como (31), ou seja, no máximo como $\exp \left(-\eta_{k} x^{2}\right)$, enquanto $\left|V\left(x_{\lambda}+\xi_{s}\right)\right|^{2}$ cresce como $\exp \left(\hbar_{\lambda} \rho x^{2}\right)$. Cada termo, nesta região, é, portanto, $O\left(\hbar_{\lambda}^{N}\right)$, para todo $N$.

Agora, para a região $\left\{|x| \leq \hbar_{\lambda}^{-1 / 2} \sigma\right\}$ consideremos o termo

$$
A=\left|\hbar_{\lambda}^{-1} V\left(x_{\lambda}+\xi_{s}\right)-\hbar_{\lambda}^{-1} V\left(\xi_{s}\right)-\hbar_{\lambda}^{-1 / 2} V^{\prime}\left(\xi_{s}\right) x-V^{\prime \prime}\left(\xi_{s}\right) \frac{x^{2}}{2}\right| .
$$

Expandindo $V(y)=V\left(x_{\lambda}+\xi_{s}\right)$ em torno de $\xi_{s}$ até o primeiro termo, e usando 
a fórmula do restc 51 .

$$
\begin{gathered}
A=\mid \hbar_{\lambda}^{-1}\left(V\left(\xi_{s}\right)+V^{\prime}\left(\xi_{s}\right) x_{\lambda}+\int_{\xi_{s}}^{x_{\lambda}+\xi_{s}}\left(x_{\lambda}+\xi_{s}-t\right) V^{\prime \prime}(t) d t\right)- \\
-\hbar_{\lambda}^{-1} V\left(\xi_{s}\right)-\hbar_{\lambda}^{-1 / 2} V^{\prime}\left(\xi_{s}\right) x-V^{\prime \prime}\left(\xi_{s}\right) \frac{x^{2}}{2} \mid \\
=\left|\hbar_{\lambda}^{-1} \int_{\xi_{s}}^{x_{\lambda}+\xi_{s}}\left(x_{\lambda}+\xi_{s}-t\right) V^{\prime \prime}(t) d t-V^{\prime \prime}\left(\xi_{s}\right) \frac{x^{2}}{2}\right| .
\end{gathered}
$$

Fazendo a mudança de variável, $u=t-\xi_{s}$ :

$$
A=\left|\hbar_{\lambda}^{-1} \int_{0}^{x_{\lambda}}\left(x_{\lambda}-u\right) V^{\prime \prime}\left(u+\xi_{s}\right) d u-V^{\prime \prime}\left(\xi_{s}\right) \frac{x^{2}}{2}\right| .
$$

E agora $u=x_{\lambda} y$ :

$$
A=\left|\hbar_{\lambda}^{-1} \int_{0}^{1} x_{\lambda}^{2}(1-y) V^{\prime \prime}\left(x_{\lambda} y+\xi_{s}\right) d y-V^{\prime \prime}\left(\xi_{s}\right) \frac{x^{2}}{2}\right| .
$$

Como $\int_{0}^{1}(1-y) d y=1 / 2$ podemos incluir o segundo termo dentro da integral incorporando o $1 / 2$ no $1-y$ (e já cancelando $\hbar_{\lambda}^{-1} \operatorname{com~o~} \hbar_{\lambda}$ de $x_{\lambda}$ ):

$$
\begin{array}{r}
A=\left|x^{2} \int_{0}^{1}(1-y)\left(V^{\prime \prime}\left(x_{\lambda} y-\xi_{s}\right)-V^{\prime \prime}\left(\xi_{s}\right)\right) d y\right| \leq \\
x^{2} \int_{0}^{1}(1-y)\left|V^{\prime \prime}\left(x_{\lambda} y-\xi_{s}\right)-V^{\prime \prime}\left(\xi_{s}\right)\right| d y .
\end{array}
$$

Utilizando a continuidade de Hölder de $V^{152}$.

$$
x^{2} \int_{0}^{1}(1-y)\left|V^{\prime \prime}\left(x_{\lambda} y-\xi_{s}\right)-V^{\prime \prime}\left(\xi_{s}\right)\right| d y \leq C x^{2+\delta} \hbar_{\lambda}^{\delta / 2}=O\left(\hbar_{\lambda}^{\delta / 2}\right) .
$$

Portanto, $\left\|W(t, 0) \psi_{a}-W_{\lambda}(t, 0) \psi_{a}\right\|=O\left(\hbar_{\lambda}^{\delta / 2}\right)$, então $W_{\lambda}(t, 0)$ converge fortemente à $W(t, 0)$ quando $\lambda \rightarrow 0$.

Resumindo os resultados: descobrimos que

$$
U\left(\hbar_{\lambda}^{-1 / 2} \alpha\right)^{*} U_{\lambda}(t)^{*} e^{i\left[r\left(q-\hbar_{\lambda}^{-1 / 2} \xi_{t}\right)+s\left(p-\hbar_{\lambda}^{-1 / 2} \pi_{t}\right)\right]} U_{\lambda}(t) U\left(\hbar_{\lambda}^{-1 / 2} \alpha\right)=
$$

\footnotetext{
${ }^{51} \mathrm{Ou}$ seja, dada $f$, expandindo-a em torno de $a$ isso significa, $f(x)=f(a)+f^{\prime}(a)(x-$ a) $+R_{1}(x)$, onde

$$
R_{1}(x)=\int_{a}^{x}(x-t) f^{\prime \prime}(t) d t
$$

${ }^{52}$ Uma função é Hölder continua se existe uma constante $C$ e $\delta$ tal que $|f(x)-f(y)| \leq$ $C|x-y|^{\delta}$.
} 


$$
=W_{\lambda}(t, 0)^{*} e^{i[r q+s p]} W_{\lambda}(t, 0) \longrightarrow W(t, 0)^{*} e^{i[r q+s p]} W(t, 0),
$$

o que prova (26). O resultado (27) segue imediatamente do que foi provado:

$$
\begin{gathered}
U\left(\hbar_{\lambda}^{-1 / 2} \alpha\right)^{*} U_{\lambda}(t)^{*} e^{i\left[r q_{\lambda}+s p_{\lambda}\right]} U\left(\hbar_{\lambda}^{-1 / 2} \alpha\right) U_{\lambda}(t) \psi-e^{i\left[r \xi_{t}+s \pi_{t}\right]} \psi \\
=U\left(\hbar_{\lambda}^{-1 / 2} \alpha\right)^{*} U_{\lambda}(t)^{*} \exp \left(\hbar_{\lambda}^{1 / 2} i\left(r\left[q-\hbar_{\lambda}^{-1 / 2} \xi_{t}\right]+s\left[p-\hbar_{\lambda}^{-1 / 2} \pi_{t}\right]\right)\right) U\left(\hbar_{\lambda}^{-1 / 2} \alpha\right) U_{\lambda}(t) \psi-\psi \mathbb{1} \\
=W_{\lambda}(t, 0)^{*} e^{\hbar_{\lambda}^{1 / 2} i(r q+s p)} W_{\lambda}(t, 0) \psi-\psi \mathbb{1} .
\end{gathered}
$$

Como $e^{\hbar_{\lambda}^{1 / 2} i(r q+s p)} \rightarrow \mathbb{1}$ e $W_{\lambda}(t, 0) \rightarrow W(t, 0)$, de modo que resta somente $\psi \mathbb{1}-\psi \mathbb{1}$, vemos que (32) converge fortemente. Isso prova o teorema.

Infinitos graus de liberdade Sabendo a formulação precisa no caso da mecânica quântica em uma dimensão, a forma e a matéria do teorema de Hepp do limite semi-clássico podemos formular o mesmo teorema para o caso de um sistema bosônico com infinitos graus de liberdade, ou seja, um sistema formado por uma álgebra CCR (de Weyl) que satisfaz os axiomas de Haag-Kastler. Os operadores, que são representações de elementos da álgebra, foram construídos em, por exemplo, 32] por Glimm e Jaffe. Para deixar clara a eventual formulação do limite semi-clássico num espaço-tempo globalmente hiperbólico (em particular de de Sitter), escreveremos o presente teorema já utilizando a linguagem algébrica mais geral, e assim, restará como dificuldade central do teorema geral a construção de operadores de evolução temporal em espaços-tempos de de Sitter.

Tomamos o estado coerente $\psi$ dado em termos do espaço de Hilbert por:

$$
\psi_{\lambda}(W(f))=\left\langle\alpha_{\lambda}|W(f)| \alpha_{\lambda}\right\rangle
$$

onde $\left|\alpha_{\lambda}\right\rangle=W\left(\hbar_{\lambda}^{-1 / 2} \alpha\right)|0\rangle$, e $\alpha=(\phi+i \pi) / \sqrt{2}$, onde $\phi$ é solução da equação de Klein-Gordon clássica com interação polinomial:

$$
\left(\square+m^{2}\right) \phi(x, t)+\sum_{n=1}^{N} n a_{n} \phi(x, t)^{n-1}=0 .
$$

Como vimos antes, equivalendo-se $\psi_{l}(W(f))=e^{i l(f)} e^{-\|f\|^{2} / 4}$ com o estado coerente convencional $W(\pi, \phi)|0\rangle$ obtemos $l(f)=\left\langle\rho_{1} G f, \phi\right\rangle-\left\langle\pi, \rho_{0} G f\right\rangle$. E se $\alpha \mapsto \hbar_{\lambda}^{-1 / 2} \alpha$, então, $l(f) \mapsto \hbar_{\lambda}^{-1 / 2} l(f)$. Assim,

$$
\psi_{\lambda}(W(f))=e^{i \hbar_{\lambda}^{-1 / 2}\langle f, u\rangle} e^{-\|f\|^{2} / 4} .
$$


Seja $\Phi(f)$ um elemento gerador da álgebra CCR, e então que satisfaça $[\Phi(f), \Phi(g)]=$ $i \sigma(f, g) \mathbb{1}$. E seja $\Phi_{\lambda}(f)=\sqrt{\hbar \lambda} \Phi(f)$, onde, novamente, $\lambda$ é um número real, que tomaremos tendendo à zero no limite semi-clássico.

Seja o hamiltoniano $H_{\lambda r}=\hbar_{\lambda} H_{0}+V_{\lambda}\left(g_{r}\right)$, onde, para $r>0, \mathscr{D}^{\prime}(\mathbb{R}) \ni g_{r} \geq 0$, $\left.g\right|_{\{x:|x| \leq r\}}=1$, e

$$
V_{\lambda}\left(g_{r}\right)=\sum_{n=1}^{N} a_{n} \int g_{r}(x): \Phi_{\lambda}(x)^{n}: d x
$$

e seja o gerador, $U_{\lambda r}(t)=\exp \left[-i t H_{\lambda r} /(\hbar \lambda)\right]$, e $\mathfrak{a}_{t}^{\lambda}$ o automorfismo na álgebra CCR que representa evolução temporal, ou seja, $\mathfrak{a}_{t}^{\lambda}(W(f))=U_{\lambda r}(t)^{*} W(f) U_{\lambda r}(t)$. Por fim, seja $Q(\alpha, x, t)$ a solução da equação de Klein-Gordon com interações polinomiais linearizada em torno de $\phi(x, t)$ :

$$
\left(\square+m^{2}\right) Q(\alpha, x, t)+\sum_{n=2}^{N} n(n-1) a_{n} \phi(x, t)^{n-2} Q(\alpha, x, t)=0 .
$$

Temos assim o seguinte teorema:

Teorema 5.2. Suponha que $\phi(x, t)$ exista para $|t|<T$, então:

$$
\lim _{\lambda \rightarrow 0} \psi_{\lambda}\left(\mathfrak{a}_{t}^{\lambda}\left(W\left(f-\phi_{\lambda}(f, t) \mathbb{1}\right)\right)\right)=e^{i Q(\alpha, f, t)}
$$

$e$

$$
\lim _{\lambda \rightarrow 0} \psi_{\lambda}\left(\mathfrak{a}_{t}^{\lambda}\left(W\left(f_{\lambda}\right)\right)\right)=e^{i \phi(f, t)}
$$

onde $f_{\lambda}=\sqrt{\hbar \lambda} f$

Exemplo Tomemos como exemplo o caso de um sistema livre: $H_{\lambda r}=$ $\hbar_{\lambda} H_{0}$, num espaço de Fock bosônico $\mathscr{H}_{F}^{+}$. O operador de evolução de uma partícula, no caso de um sistema livre, não depende de $\lambda: U_{t}=\exp \left(-i t H_{0}\right)$. A extensão para várias partículas é, naturalmente, dada pelo operador

$$
\left.\Gamma\left(U_{t}\right)\right|_{\mathscr{H}_{n}^{+}}=\bigotimes_{k=1}^{n} U_{t}
$$

já que, como mostra nos mostra Reed e Simon em [26] $\Gamma\left(U_{t}\right)=\exp \left(-i t d \Gamma\left(H_{0}\right)\right)$. E ainda,

$$
\Gamma\left(U_{t}\right)^{-1} \Phi(f) \Gamma\left(U_{t}\right)=\Phi\left(U_{t}^{-1} f\right) .
$$

\footnotetext{
${ }^{53}$ Isso significa que, tomando $W(f)=\exp (i \Phi(f))$ e como $\sqrt{\hbar \lambda} \in \mathbb{R}, W\left(f_{\lambda}\right)=$ $\exp \left(i \Phi_{\lambda}(f)\right)$.
} 
A demonstração disso é uma simples conta, e é idêntica a feita em [26]. Portanto,

$$
\mathfrak{a}_{t}^{\lambda}(W(f))=\Gamma\left(U_{t}\right)^{-1} W(f) \Gamma\left(U_{t}\right)=W\left(U_{t}^{-1} f\right)
$$

Então,

$$
\psi_{\lambda}\left(\mathfrak{a}_{t}^{\lambda}\left(W\left(f_{\lambda}\right)\right)\right)=\psi_{\lambda}\left(W\left(U_{t}^{-1} f_{\lambda}\right)\right)=e^{i \hbar_{\lambda}^{-1 / 2}\left\langle U_{t}^{-1} \hbar_{\lambda}^{1 / 2} f, u\right\rangle} e^{-\hbar_{\lambda}^{1 / 2}\left\|U_{t}^{-1} f\right\|^{2} / 4} .
$$

Como $U_{t}$ é unitária:

$$
\psi_{\lambda}\left(\mathfrak{a}_{t}^{\lambda}\left(W\left(f_{\lambda}\right)\right)\right)=e^{i\left\langle f, U_{t} u\right\rangle} e^{-\hbar_{\lambda}^{1 / 2}\|f\|^{2} / 4} \Rightarrow \lim _{\lambda \rightarrow 0} \psi_{\lambda}\left(\mathfrak{a}_{t}^{\lambda}\left(W\left(f_{\lambda}\right)\right)\right)=e^{i\left\langle f, U_{t} u\right\rangle} .
$$

Ou seja, $u$, que é a solução da equação de Klein-Gordon homogênea é evoluída por $U_{t}: u(t)$. Em sentido distribucional temos, então, $\left\langle f, U_{t} u\right\rangle=u(f, t)$, como de fato exige o teorema.

É proveitoso ainda olhar para esse exemplo de outra forma. No caso em que o espaço-tempo é curvo, como o espaço-tempo de de Sitter, devemos de alguma forma evoluir os operadores que representam a álgebra sem fazer referencia direta ao Hamiltoniano. O axioma 2 dos axiomas de Haag-Kastler nos dizem como isometrias entre espaços-tempos são implementadas nas álgebras como isomorfismos. Caso a transformação isométrica seja dentro do espaçotempo - e, assim, no caso de Minkowski, elas são transformações de Poincaré $g, g x=\Lambda x+a$ - então o isomorfismo garantido pelos axiomas é um automorfismo, digamos, $\mathfrak{a}_{g}: \mathscr{A} \rightarrow \mathscr{A}$, que age da seguinte forma:

$$
\mathfrak{a}_{g}(\mathscr{A}(\mathcal{O}))=\mathscr{A}(g \mathcal{O}) .
$$

Como $\mathscr{A}(\mathcal{O})$ é a álgebra gerada por

$$
\left\{W(f): f \in \mathfrak{F}_{0}(\mathscr{M}), \quad \operatorname{supp}(f) \subset \mathcal{O}\right\}
$$

A transformação $\mathcal{O} \mapsto g \mathcal{O}$ induz no espaço de Hilbert formado pelas funções $f$ a transformação $U(\Lambda, y)$. Assim, $\mathfrak{a}_{g}(W(f))=W(U(\lambda, y) f)$. Como queremos saber como translações temporais são implementadas tomamos $\Lambda=1$ e $y=$ $(t, 0,0,0)$, e neste caso $U(1, y)=\exp (i t H)=U_{t}^{-1}$, e recuperamos o caso anterior. A equação do teorema que nos garante o limite semi-clássico (para campos livres) pode ser reescrita:

$$
\lim _{\lambda \rightarrow 0} \psi_{\lambda}\left(\mathfrak{a}_{t}\left(W\left(f_{\lambda}\right)\right)\right)=e^{i u(f, t)},
$$

onde $\mathfrak{a}_{t}$ é o automorfismo induzido pela transformação de Poincaré $(1, y), y=$ $(t, 0,0,0)$. 
6 O Espaço de de Sitter 
Há diversas formas de se definir o espaço-tempo de de Sitter, mas a forma mais simples (que é, claro, equivalente à outras formas, vide, e.g., [11]) é identificá-lo com uma subvariedade no espaço-tempo de Minkowskit a saber, a subvariedade definida pelo hiperboloide:

$$
d S=\left\{x \in \mathbb{R}^{3}: x^{2}=-r^{2}\right\},
$$

onde $x^{2}=x_{\mu} x^{\mu}=\left(x^{0}\right)^{2}-\left(x^{1}\right)^{2}-\left(x^{2}\right)^{2}$. Dois pontos $x, y \in d S$ possuem uma separação tipo-tempo se $(x-y)^{2}=\left(x^{0}\right)^{2}+\left(y^{0}\right)^{2}-2 x^{0} y^{0}-\left(x^{1}\right)^{2}-$ $\left(y^{1}\right)^{2}+2 x^{1} y^{1}-\left(x^{2}\right)^{2}-\left(y^{2}\right)^{2}+2 x^{2} y^{2}=-2 r^{2}-2 x_{\mu} y^{\mu}>0 \therefore x^{\mu} y_{\mu}<-r^{2}$. Da mesma forma, $x$ e $y$ possuem uma separação tipo-luz, causal, tipo-tempo se $x . y=-r^{2}, x . y \leq 0, x . y>0$.

Um subconjunto de particular importância é o circulo no tempo zero,

$$
S^{1}=\{(0, r \sin \theta, r \cos \theta), \theta \in[-\pi / 2,3 \pi / 2)\},
$$

que em verdade é uma superfície de Cauchy em $d S$.

As isometrias em $d S$ são dadas pelo grupo de Lorentz $O(1,2)$,

$$
O(1,2)=\left\{\Lambda \in G L(\mathbb{R}, 3): \Lambda^{-1}=\eta \Lambda^{T} \eta\right\},
$$

onde $\eta$ é a métrica do espaço de Minkowski $\left(\mathbb{R}^{1+2}, \eta\right)$. É comum decompor o grupo de Lorentz em componentes disjuntas, $O(1,2)_{+}^{\uparrow}, O(1,2)_{+}^{\downarrow}, O(1,2)_{-}^{\uparrow} \mathrm{e}$ $O(1,2)_{-}^{\downarrow}$. Apenas o primeiro destes é um subgrupo de $O(1,2)$, e é geralmente denotado também como $S O_{0}(1,2)$, e é definido por:

$$
S O_{0}(1,2)=\left\{\Lambda \in O(1,2): \operatorname{det}(\Lambda)=1,(\Lambda)_{0}^{0}>1\right\} .
$$

O grupo $S O_{0}(1,2)$ é formado por uma rotação sobre o eixo $x^{0}, R(\alpha)$ e dois boosts, $\Lambda_{1}(t), \Lambda_{2}(s)$ :

$$
\begin{gathered}
R(\alpha)=\left(\begin{array}{ccc}
1 & 0 & 0 \\
0 & \cos (\alpha) & -\sin (\alpha) \\
0 & \sin (\alpha) & \cos (\alpha)
\end{array}\right), \Lambda_{1}(t)=\left(\begin{array}{ccc}
\cosh (t) & 0 & \sinh (t) \\
0 & 1 & 0 \\
\sinh (t) & 0 & \cosh (t)
\end{array}\right) \\
\Lambda_{2}(s)=\left(\begin{array}{ccc}
\cosh (s) & \sinh (s) & 0 \\
\sinh (s) & \cosh (s) & 0 \\
0 & 0 & 1
\end{array}\right) .
\end{gathered}
$$

Para $t$ ou $s$ pequenos podemos aproximar os boosts $\Lambda_{1}(t)$ e $\Lambda_{2}(s)$ às matrizes:

$$
\Lambda_{1}(t) \approx\left(\begin{array}{ccc}
1 & 0 & t \\
0 & 1 & 0 \\
t & 0 & 1
\end{array}\right), \Lambda_{2}(s) \approx\left(\begin{array}{ccc}
1 & s & 0 \\
s & 1 & 0 \\
0 & 0 & 1
\end{array}\right)
$$

\footnotetext{
${ }^{54}$ Trataremos aqui espaços-tempos de de Sitter em 1+1, então o espaço de Minkowski será $\left(\mathbb{R}^{1+2}, \eta\right)$.
} 
Essas matrizes correspondem a evoluções temporais em torno de $x^{0}=0$ :

$$
\left(\begin{array}{lll}
1 & 0 & t \\
0 & 1 & 0 \\
t & 0 & 1
\end{array}\right)\left(\begin{array}{c}
0 \\
x^{1} \\
x^{2}
\end{array}\right)=\left(\begin{array}{c}
x^{2} t \\
x^{1} \\
x^{2}
\end{array}\right)
$$

Ocorre um fenômeno análogo para $\Lambda_{2}(s)\left(0, x^{1}, x^{2}\right)$, mas ao invés de $x^{0^{\prime}}=x^{2} s$ temos $x^{0^{\prime}}=x^{1} s$, o que de fato é equivalente, já que $\left(x^{1}\right)^{2}+\left(x^{2}\right)^{2}=r^{2}$.

Ou seja, para regiões $\mathcal{O}$ próximas da origem e para $t$ pequeno, $\Lambda_{1}(t) \mathcal{O}$ representa uma evolução temporal da região $\mathcal{O}$.

Por outro lado, como $\Lambda \in S O(1,2), \Lambda^{-1}=\eta \Lambda^{T} \eta$, então,

$$
\Lambda_{1}(t)^{-1}=\left(\begin{array}{ccc}
\cosh (t) & 0 & -\sinh (t) \\
0 & 1 & 0 \\
-\sinh (t) & 0 & \cosh (t)
\end{array}\right)=\Lambda_{1}(-t) .
$$

Assim, para $t$ pequeno,

$$
\Lambda_{1}(t)^{-1} \approx\left(\begin{array}{ccc}
1 & 0 & -t \\
0 & 1 & 0 \\
-t & 0 & 1
\end{array}\right)
$$

\subsection{Equação de Onda e Quantização}

O espaço-tempo de de Sitter é globalmente hiperbólico. Há, portanto, uma solução global para o problema de Cauchy para $P=\square+\mu^{2}$, onde $\mu$ é um termo de massa, o qual assumimos maior que zero, com os dados $\left(f, u_{0}, u_{1}\right)$. Ou seja, há um único $u \in \mathfrak{F}(d S)$ que satisfaz o sistema

$$
\left\{\begin{array}{l}
\left(\square+\mu^{2}\right) u=f \\
\rho_{0}(u)=u_{0} \\
\rho_{1}(u)=u_{1}
\end{array}\right.
$$

Onde $\rho_{i}: \mathfrak{F}(d S) \rightarrow \mathfrak{F}\left(S^{1}\right)$. E existem ainda operadores de Green $G_{ \pm}:$ $\mathfrak{F}_{0}(d S) \rightarrow \mathfrak{F}(d S)$ que geram soluções de $P u=f$, já que, $P \circ G_{ \pm}=\mathbb{1}$, assim, $u=G_{ \pm} f$. E claro, $u=G f$, onde $G=G_{+}-G_{-}$, é solução da equação de Klein-Gordon homogênea: $\left(\square+\mu^{2}\right) u=0$.

Quantizar esse campo é apenas um caso particular do processo descrito na seção (4.2). Consideramos as álgebras que satisfazem

$$
W\left(f_{1}, f_{2}\right) W\left(g_{1}, g_{2}\right)=e^{-i\left(\left\langle f_{1}, g_{2}\right\rangle-\left\langle f_{2}, g_{1}\right\rangle\right)} W\left(f_{1}+f_{2}, g_{1}+g_{2}\right), \quad f_{i}, g_{i} \in \mathfrak{F}_{0}\left(S^{1}\right),
$$


onde $\langle f, g\rangle=\int_{S^{1}} f g d V$. E definimos ainda

$$
W(f)=W\left(\rho_{1} G f, \rho_{0} G f\right), \quad f \in \mathfrak{F}_{0}(d S),
$$

que satisfazem as relações características de álgebras CCR-Weyl:

$$
W(f) W(g)=e^{-i\langle f, G g\rangle / 2} W(f+g),
$$

e $\langle f, G g\rangle=\sigma(f, g)$ é uma forma simplética. Portanto, a rede de álgebra $\mathscr{A}(\mathcal{O})$, onde $\mathcal{O} \subset d S$ é uma região limitada e aberta, gerada por

$$
\{W(f): \operatorname{supp} f \subset \mathcal{O}\}
$$

junto com a álgebra quasi-local $\mathscr{A}$ satisfazem os axiomas de Haag-Kastler generalizados.

Há uma representação do grupo de isometrias no espaço simplético: para cada $\Lambda \in O(1,2), U_{\Lambda}: \mathfrak{F}_{0}(d S) \rightarrow \mathfrak{F}_{0}(d S)$ definido através do pull-back por:

$$
U_{\Lambda} f=\Lambda^{*} f, \text { i.e., }\left(U_{\Lambda} f\right)(x)=f\left(\Lambda^{-1} x\right), \quad \forall \Lambda \in O(1,2) .
$$

É fácil verificar que 38 é de fato uma representação do grupo $O(1,2)$ em $\mathfrak{F}_{0}(d S){ }^{55}$

Do axioma (2) (vide seção (4.1)) temos que toda isometria induz na rede de álgebras um *-isomorfismo, e neste caso $S O_{0}(1,2)$ induz um *-automorfismo $\alpha_{\Lambda}: \alpha_{\Lambda}(\mathscr{A}(\mathcal{O}))=\mathscr{A}(\Lambda \mathcal{O})$.

Como $\mathcal{O} \mapsto \Lambda \mathcal{O}$ induz em $\mathfrak{F}_{0}(d S)$ a transformação $U_{\Lambda}$ há um automorfismo induzido na álgebra $\alpha_{\Lambda}: \mathscr{A} \rightarrow \mathscr{A}$ dada por

$$
\left(\alpha_{\Lambda} W\right)(f)=W\left(U_{\Lambda}^{-1} f\right) .
$$

Tomando $\Lambda$ como sendo $\Lambda_{1}(t)$ para $t$ pequeno, agindo em regiões $\mathcal{O}$ definidas na origem, $x^{0}=0$ temos uma evolução temporal, mas restrita à pequenas regiões em torno da origem ${ }^{56}$ Ou seja, temos uma dinâmica mesmo que restrita, e isso nos permitirá não só fazer uma equivalência estática das álgebras com os resultados clássicos, mas ainda mais ver que essa equivalência permanece sob evoluções temporais.

Importa ainda saber o que significa $U_{\Lambda} u$, onde $u$ é uma solução do problema de Cauchy da equação de Klein-Gordon com os dados $u_{i}=\rho_{i}(u)$ :

\footnotetext{
${ }^{55}$ Isto é, que $U_{\Lambda}$ satisfaz, para cada $\Lambda_{i}, U_{\Lambda_{1}} U_{\Lambda_{2}}=U_{\Lambda_{1} \Lambda_{2}}, U_{\mathbb{1}}=1, U_{\Lambda^{-1}}=U_{\Lambda}^{-1}$.

${ }^{56}$ É claro que poderíamos tomar $\Lambda$ como sendo uma composição de $\Lambda_{1}$ ou $\Lambda_{2}$ com $R(\alpha)$.
} 
$\left(U_{\Lambda} u\right)(x)$ representa a solução $u$ calculada em um domínio transformado, ou seja, $\left(U_{\Lambda} u\right)(x)=u\left(\Lambda^{-1} x\right)$, assim,

$$
\left(U_{\Lambda} u\right)(x) \neq 0 \Leftrightarrow u\left(\Lambda^{-1} x\right) \neq 0 \Leftrightarrow \Lambda^{-1} x \in \operatorname{supp}(u) ;
$$

então, $x \in \Lambda \operatorname{supp}(u)$. Ou seja, o suporte de $U_{\Lambda} u$ é o suporte de $u$ transformado por $\Lambda$.

Então, para $t$ pequeno e $u$ com o suporte compacto em pequenas regiões perto da origem $U_{\Lambda_{1}(t)} u$ representa a solução da equação de Klein-Gordon evoluída temporalmente, $\operatorname{supp}(u) \ni\left(0, x^{1}, x^{2}\right) \mapsto\left(x_{t}^{0}, x^{1}, x^{2}\right) \in \operatorname{supp}\left(U_{\Lambda_{1}(t)} u\right)$, onde $x_{t}^{0}=t x^{2}$ é também próximo de zero, de modo que neste caso é possível calcular como os dados de Cauchy são modificados, $u_{i}^{\prime}=\rho_{i}\left(U_{\Lambda_{1}(t)} u\right)$, já que

$$
U_{\Lambda_{1}(t)} u=u\left(x^{0}, x^{1}, x^{2}\right) \approx u\left(0, x^{1}, x^{2}\right)+\left.x^{0} \frac{\partial u}{\partial x^{0}}\right|_{x^{0}=0}=u_{0}+x^{0} u_{1} .
$$

Então,

$u_{0}^{\prime}=\rho_{0}\left(u_{0}+x^{0} u_{1}\right)=u_{0}+x^{0} u_{1}, \quad u_{1}^{\prime}=\left.\frac{\partial}{\partial x^{0}}\left(u_{0}+x^{0} u_{1}\right)\right|_{x^{0}=0}=u_{1}+\left.x^{0} \frac{\partial^{2} u}{\partial x^{0^{2}}}\right|_{x^{0}=0}$.

É provado em [11] que a restrição da métrica de Minkowski ao hiperboloide $d S$ é

$$
\left.\left(d t^{2}-\sum_{i}\left(d x^{i}\right)^{2}\right)\right|_{d S}=\left(d x^{0}\right)^{2}-r^{2} \cosh ^{2}\left(\frac{x^{0}}{r}\right) d \theta^{2} .
$$

Assim, $\sqrt{|g|}=r \cosh \left(x^{0} / r\right) \mathrm{e}$

$$
\square=\frac{1}{r \cosh \left(x^{0} / r\right)}\left(\partial_{0}\left(r \cosh \left(x^{0} / r\right) \partial_{0}\right)+\partial_{\theta}\left(g^{\theta \theta} r \cosh \left(x^{0} / r\right) \partial_{\theta}\right)\right) .
$$

Nomeando o último termo de $P_{\theta}$ :

$$
\square+m^{2}=\tanh \left(x^{0} / r\right) \frac{\partial}{\partial x^{0}}+\frac{\partial^{2}}{\partial\left(x^{0}\right)^{2}}+P_{\theta}+m^{2} .
$$

Como $u$ é solução da equação de Klein-Gordon

$$
\left(\square+m^{2}\right) u=0 \therefore \frac{\partial^{2}}{\partial\left(x^{0}\right)^{2}} u=-\tanh \left(x^{0} / r\right) \frac{\partial}{\partial x^{0}} u-P_{\theta} u,
$$

então,

$$
\left.\left(\frac{\partial^{2}}{\partial\left(x^{0}\right)^{2}} u\right)\right|_{x^{0}=0}=P_{\theta} u_{0}
$$




\subsection{Limite Semi-Clássico}

Como feito na seção (5) definimos o estado coerente $\psi_{l}: \mathscr{A} \rightarrow \mathbb{C}$ na álgebra CCR-Weyl que são as álgebras de observáveis locais que satisfazem as relações canônicas de comutação, dado por $\psi_{l}(W(f))=\exp (i l(f)) \exp \left(-\|f\|^{2} / 4\right)$, onde $l$ é definido através de duas funções $\left(u_{0}, u_{1}\right)$ suaves definidas na superfície de Cauchy por

$$
l(f)=\left\langle\rho_{1} G f, u_{0}\right\rangle-\left\langle u_{1}, \rho_{0} G f\right\rangle .
$$

Tomamos o estado coerente $\psi_{l}$ centrado em $\hbar_{\lambda}^{-1 / 2}$ ou seja, temos como em (34):

$$
\psi_{\lambda}(W(f))=e^{i \hbar_{\lambda}^{-1 / 2} l(f)} e^{-\|f\|^{2} / 4}
$$

Uma versão fraca do teorema de Hepp (a saber, o teorema que já era conhecido de Ehrenfest) garante que elementos $W(f)$ da álgebra local que representa observáveis definidos localmente correspondem à soluções da equação de Klein-Gordon definidas também no mesmo loca 57

Teorema 6.1. Seja $\psi_{\lambda}$ como definido por (39), e seja u a solução do problema de Cauchy da equação de Klein-Gordon homogênea com os dados de Cauchy $\left(u_{0}, u_{1}\right)$. Então,

$$
\lim _{\lambda \rightarrow 0} \psi_{\lambda}\left(W\left(f_{\lambda}\right)\right)=e^{i u(f)}
$$

Demonstração. A prova deste teorema é meramente uma conta:

$$
\psi_{\lambda}\left(W\left(f_{\lambda}\right)\right)=\exp \left(i \hbar_{\lambda}^{-1 / 2} l\left(\hbar_{\lambda}^{1 / 2} f\right)\right) \exp \left(\left\|\hbar_{\lambda}^{1 / 2} f\right\|^{2} / 4\right) .
$$

Como $\left\|\hbar_{\lambda}^{1 / 2} f\right\|=\hbar_{\lambda}\|f\|$, a segunda exponencial tende a um quando $\lambda$ tende a zero. Por outro lado,

$$
l\left(\hbar_{\lambda}^{1 / 2} f\right)=\left\langle\rho_{1} G \hbar_{\lambda}^{1 / 2} f, u_{0}\right\rangle-\left\langle u_{1}, \rho_{0} G \hbar_{\lambda}^{1 / 2} f\right\rangle=\hbar_{\lambda}^{1 / 2} l(f) .
$$

Então,

$$
\lim _{\lambda \rightarrow 0} \psi_{\lambda}\left(W\left(f_{\lambda}\right)\right)=\exp (i l(f)) .
$$

E, invocando (18), $l(f)=\langle u, f\rangle=u(f)$.

\footnotetext{
${ }^{57}$ Já que $W(f) \in\{W(f): \operatorname{supp}(f) \subset \mathcal{O}\}$, então, uma distribuição (que como veremos é solução da equação de Klein-Gordon) calculada para $f$ está, em essência, definida em $\operatorname{supp} f$ :

$$
u(f)=\int_{\mathscr{M}} f(x) u(x) d V=\int_{\operatorname{supp} f \subset \mathcal{O}} f(x) u(x) d V .
$$
}


Uma versão um pouco mais geral deste teorema que é por sua vez a generalização do teorema de Hepp é o caso no qual consideramos transformações nas regiões locais sobre as quais as álgebras locais estão definidas. Ou seja, a isometria $\Lambda$ que age, digamos, numa região $\mathcal{O}$, induz na álgebra $\mathscr{A}(\mathcal{O})$ um automorfismo $\alpha_{\Lambda}: \mathscr{A}(\mathcal{O}) \rightarrow \mathscr{A}(\Lambda \mathcal{O})$ e no espaço simplético a representação $U_{\Lambda}$. Queremos saber ao que, através do estado coerente $\psi_{\lambda}$, o ente quântico que é transformado - e o teorema abaixo nos garante que ele corresponderá à solução da equação de Klein-Gordon implícita em $\psi_{\lambda}$ mas transformado no sentido que descrevemos na seção anterior, viz., ele será $U_{\Lambda} u$. Em particular, se $\Lambda=\Lambda_{1}(t)$ e a região $\mathcal{O}$ for próxima da origem e $t$ muito pequeno, isso corresponderá à uma evolução temporal e $U_{\Lambda_{1}(t)} u$ serpa como o descrito na seção anterior.

Teorema 6.2. Seja $\psi_{\lambda}$ como definida acima em (39), com $u_{0}$ e $u_{1}$, definidos na superfície de Cauchy $S^{1}$. E seja u a solução do problema de Cauchy da equação de Klein-Gordon com os dados $\left(u_{0}, u_{1}\right)$. Seja $\alpha_{\Lambda}: \mathscr{A} \rightarrow \mathscr{A}$ o automorfismo induzido pela isometria $\Lambda \in S O(1,2)$. Então,

$$
\lim _{\lambda \rightarrow 0} \psi_{\lambda}\left(\left(\alpha_{\Lambda} W\right)\left(f_{\lambda}\right)\right)=e^{i\left(U_{\Lambda} u\right)(f)} .
$$

Em particular, se $\Lambda=\Lambda_{1}(t)$ e supp $(f) \subset \mathcal{O}$ é próximo da origem (e.g., $\mathcal{O} \ni$ $\left.\left(x^{0}, x^{1}, x^{2}\right)=\left(0, x^{1}, x^{2}\right)\right)$ e t for pequeno, então $U_{\Lambda_{1}(t)} u$ é representado funcionalmente por $u\left(x^{0}, x^{1}, x^{2}\right)$, onde $x^{0}=t x^{2}$, e $\operatorname{supp}\left(U_{\Lambda_{1}(t)} u\right)=\Lambda_{1}(t) \operatorname{supp}(u)$.

Demonstração. A prova deste teorema é simples: consiste apenas em uma conta:

$$
\psi_{\lambda}\left(\left(\alpha_{\Lambda} W\right)\left(f_{\lambda}\right)\right)=\psi_{\lambda}\left(W\left(U_{\Lambda}^{-1} f_{\lambda}\right)\right)=e^{i \hbar_{\lambda}^{-1 / 2}\left\langle\hbar_{\lambda}^{1 / 2} U_{\Lambda}^{-1} f, u\right\rangle} e^{-\hbar_{\lambda}^{1 / 2}\left\|U_{\Lambda}^{-1} f\right\|^{2} / 4} .
$$

A segunda exponencial tende à 1 à medida que $\lambda$ tende à 0 , já que sua única dependência em $\lambda$ ocorre de forma explicita como a simples multiplicação por $\sqrt{\lambda \hbar}$. Vejamos a primeira exponencial:

$$
\left\langle U_{\Lambda}^{-1} f, u\right\rangle=\int\left(U_{\Lambda}^{-1} f\right)(x) u(x) d V=\int f(\Lambda x) u(x) d V .
$$

Fazendo a transformação $x \mapsto \Lambda x, d V \mapsto d V$ :

$$
\left\langle U_{\Lambda}^{-1} f, u\right\rangle=\int f(x) u\left(\Lambda^{-1} x\right) d V=\left\langle f, U_{\Lambda} u\right\rangle
$$


Portanto,

$$
\lim _{\lambda \rightarrow 0} \psi_{\lambda}\left(\left(\alpha_{\Lambda} W\right)\left(f_{\lambda}\right)\right)=\exp \left(i\left\langle f, U_{\Lambda} u\right\rangle\right)=\exp \left(i\left(U_{\Lambda} u\right)(f)\right) .
$$




\subsection{Observações e Conclusão}

O teorema acima não depende, no final, do particular vácuo $\exp \left(-\|f\|^{2} / 4\right)$, ou seja, ele não depende da estrutura complexa $J$. É precisamente a ambiguidade na estrutura complexa que geram representações da álgebra em espaços de Hilbert não equivalentes, assim este teorema independe do espaço de Hilbert e essa arbitrariedade na escolha da representação desaparece no limite clássico - como de fato seria o esperado.

A interpretação deste teorema tendo em vista sua origem ([21]) é bem direta: os elementos $W(f)$ da álgebra local $\mathscr{A}(\mathcal{O})$ representam - quando realizados em um espaço de Hilbert - a posição exponenciada como um observáve ${ }^{58}$, e $\psi_{\lambda}(W(f))$ é esse observável calculado no estado coerente centrado em torno de $\hbar_{\lambda}^{-1 / 2} \alpha$, onde $\alpha$ é uma combinação dos dados de Cauchy (os valores iniciais), $\alpha=\left(u_{0}+i u_{1}\right) / \sqrt{2}$. E à medida que $\lambda$ tende à zero (o que equivale "ver" a constante de Planck como muito pequena, ou seja, a tradicional forma de voltar ao mundo clássico), esse valor esperado $\psi_{\lambda}(W(f))$ tende à uma (exponencial da) solução não-quântica da equação de Klein-Gordon em um sentido distribucional precisamente onde o observável está definido. O teorema nos garante que essa equivalência é mantida quando o observável é evoluído (ao menos em pequenos intervalos de tempo): obtém-se desta evolução a solução não-quântica também evoluída.

Este resultado pode ser, à primeira vista, acusado de ser um resultado trivial: em primeiro lugar, partimos de um resultado clássico e passamos para um resultado quântico e então voltamos ao resultado clássico, mas este já está pressuposto no resultado quântico; em segundo lugar, no estado coerente já está implícita a solução clássica da equação de Klein-Gordon, recuperá-la a partir deles é um processo trivial.

Sed contra, do ponto de vista quântico esse resultado nos diz de fato algo novo. Uma certa primazia deve ser concedida ao ponto de vista quântico: o mundo já deve ser quântico antes de ser clássico (se essa correspondência existir). O que a teoria quântica de campos algébrica pretende fazer é precisamente oferecer uma teoria cujos objetos primários são já entidades quânticas (sem sequer referencia necessária à um espaço de Hilbert) que satisfazem alguns axiomas. O processo de "quantização" descrito na seção (4) é em verdade uma catacrese: o que se faz ali (explicitamente na enunciação dos axiomas, (4.1)) não é passar de um esquema clássico para um esquema quântico, mas já dar o esquema quântico. E o que se faz na seção 4.2 é mais um procedimento

\footnotetext{
${ }^{58} W(f)$ é usualmente denotado por $\exp (i \Phi(f))$.
} 
pratico do que teórico: apenas olhamos para operadores que satisfazem a equação de Klein-Gordon e uma relação de comutação e deles abstraímos uma forma, e esta obtida abandonamos o objeto que era, por assim dizer, expressão da forma.

Uma correspondência ao mundo clássico, uma "volta" que já era desejada na mecânica quântica não relativística - de fato, Hepp diz que a procura dessa correspondência é tão antiga quanto a própria mecânica quântica- é, pois, aqui ainda mais desejada e é, por assim dizer, uma novidade.

Em relação à segunda objeção: de fato a solução da equação já está implícita no estado coerente, mas só no sentido de que uma solução já está implícita dada duas funções definidas em uma superfície de Cauchy. Assim como no trabalho de Hepp (e nos teoremas de equivalência anteriores) as correspondências já estão implícitas no operador de deslocamento que define os estados coerentes, já que esses operadores são definidos através de duas funções no espaço de fases clássico. O que importa observar é que, estritamente falando, os estados coerentes não contém em si a solução da equação de Klein-Gordon, mas somente duas funções definidas numa superfície de Cauchy (tão arbitrarias quanto $\alpha$ que define o operador de deslocamento $U(\alpha)=\exp \left(\alpha a^{*}-\bar{\alpha} a\right)$ no caso dos estados coerentes tradicional). 


\section{Referências}

[1] H. Araki. Mathematical Theory of Quantum Fields. International series of monographs on physics. Oxford University Press, 1999.

[2] C. Bär and K. Fredenhagen. Quantum Field Theory on Curved Spacetime: Concepts and Mathematical Foundations. Lect. Notes Phys. 786. Springer-Verlag, 2009.

[3] C. Bär, N. Ginoux, and F. Pfäffle. Wave Equations on Lorentzian Manifolds and Quantization. ESI lectures in mathematics and physics. European Mathematical Society, 2007.

[4] J. C. A. Barata. Curso de física-matemática. http://denebola.if. usp.br/ jbarata/Notas_de_aula/capitulos.html, Fevereiro 2014.

[5] J. C. A. Barata, C. D. Jäkel, and J. Mund. The $\mathscr{P}(\varphi)_{2}$ Model on the de Sitter Space. ArXiv e-prints, Nov. 2013.

[6] A. N. Bernal and M. Sanchez. On Smooth Cauchy hypersurfaces and Geroch's splitting theorem. Commun.Math.Phys., 243:461-470, 2003.

[7] A. N. Bernal and M. Sanchez. Smoothness of time functions and the metric splitting of globally hyperbolic space-times. Commun.Math.Phys., 257:43-50, 2005.

[8] A. N. Bernal and M. Sanchez. Further results on the smoothability of Cauchy hypersurfaces and Cauchy time functions. Lett.Math.Phys., 77:183-197, 2006.

[9] O. Bratteli and D. Robinson. Operator Algebras and Quantum Statistical Mechanics 1. Operator Algebras and Quantum Statistical Mechanics. Springer, 1987.

[10] O. Bratteli and D. Robinson. Operator Algebras and Quantum Statistical Mechanics 2. Number v. 2 in Operator Algebras and Quantum Statistical Mechanics 2: Equilibrium States. Models in Quantum Statistical Mechanics. Springer, 1997.

[11] Y. Choquet-Bruhat. General Relativity and the Einstein Equations. Oxford Mathematical Monographs. OUP Oxford, 2008.

[12] R. Clifton and H. Halvorson. Are Rindler quanta real? Inequivalent particle concepts in quantum field theory. Brit.J.Phil.Sci., 52:417-470, 2001. 
[13] J. Dimock. Algebras of local observables on a manifold. Communications in Mathematical Physics, 77(3):219-228, 1980.

[14] J. Dimock. Quantum Mechanics and Quantum Field Theory: A Mathematical Primer. Cambridge University Press, 2011.

[15] P. Ehrenfest. Bemerkung über die angenäherte gültigkeit der klassischen mechanik innerhalb der quantenmechanik. Zeitschrift für Physik, 45(78):455-457, 1927.

[16] F. Friedlander. The Wave Equation on a Curved Space-time. Cambridge monographs on mathematical physics. Cambridge University Press, 1975.

[17] R. Haag. Local Quantum Physics: Fields, Particles, Algebras. Theoretical and Mathematical Physics. Springer London, Limited, 2012.

[18] R. Haag and D. Kastler. An algebraic approach to quantum field theory. Journal of Mathematical Physics, 5(7):848-861, 1964.

[19] H. Halvorson and M. Mueger. Algebraic Quantum Field Theory. ArXiv Mathematical Physics e-prints, Feb. 2006.

[20] S. Hawking and G. Ellis. The Large Scale Structure of Space-Time. Cambridge Monographs on Mathematical Physics. Cambridge University Press, 1973.

[21] K. Hepp. The classical limit for quantum mechanical correlation functions. Communications in Mathematical Physics, 35(4):265-277, 1974.

[22] L. Hörmander. The analysis of linear partial differential operators: Distribution theory and Fourier analysis. Springer Study Edition. SpringerVerlag, 1990.

[23] M. Jammer. The conceptual development of quantum mechanics. International series in pure and applied physics. McGraw-Hill, 1966.

[24] R. Kadison and J. Ringrose. Fundamentals of the Theory of Operator Algebras. Fundamentals of the Theory of Operator Algebras. American Mathematical Society, 1998.

[25] B. O'Neill. Semi-Riemannian Geometry With Applications to Relativity. Pure and Applied Mathematics. Elsevier Science, 1983. 
[26] M. Reed and B. Simon. Methods of Modern Mathematical Physics, Vol. 2. Number v. 2 in Fourier Nanlysis, Self-adjointness. Academic Press, 1975 .

[27] M. Reed and B. Simon. Methods of Modern Mathematical Physics, Vol. 1. Number v. 1 in Methods of Modern Mathematical Physics. Academic Press, 1980.

[28] W. Rudin. Functional Analysis. International series in pure and applied mathematics. McGraw-Hill, 2006.

[29] L. Ruetsche. Interpreting Quantum Field Theory. Philosophy of Science, 69(2):348-378, 2002.

[30] E. Schrödinger. Collected Papers on Wave Mechanics: Third Edition: AMS Chelsea Publishing Series. AMS Chelsea Pub., 1982.

[31] I. Segal. Postulates for General Quantum Mechanics. Annals of Mathematics, 48(4):930-948, 1947.

[32] R. Streater and L. M. Society. Mathematics of Contemporary Physics: Instructional Conference (NATO Advanced Study Institute), London, Aug./Sept. 1971, Proceedings. Academic Press, 1972.

[33] R. Streater and A. Wightman. PCT, Spin and Statistics, and All that. Landmarks in Physics. Princeton University Press, 2000.

[34] R. Wald. General Relativity. University of Chicago Press, 2010.

[35] R. M. Wald. The Formulation of Quantum Field Theory in Curved Spacetime. 2009.

[36] S. Weinberg. Gravitation and cosmology: principles and applications of the general theory of relativity. Wiley, 1972.

[37] S. YAMAGAMI. Geometry of coherent states of ccr algebras. Infinite Dimensional Analysis, Quantum Probability and Related Topics, 15(02):1250009, 2012.

[38] K. Yoshida. Functional Analysis. Classics in mathematics. Springer, 1980 . 